\title{
Transcriptomic and metabolite analyses of Cabernet Sauvignon grape berry development
} Laurent G Deluc ${ }^{1}$, Jérôme Grimplet ${ }^{1}$, Matthew D Wheatley ${ }^{1}$, Richard L Tillett ${ }^{1}$, David R Quilici ${ }^{1}$, Craig Osborne ${ }^{2}$, David A Schooley ${ }^{1}$, Karen A Schlauch ${ }^{3}$, John C Cushman ${ }^{1}$ and Grant R Cramer*1

Address: ${ }^{1}$ Department of Biochemistry and Molecular Biology, University of Nevada, Reno, Nevada 89557-0014, USA, ${ }^{2}$ Department of Animal Biotechnology, University of Nevada, Reno, NV 89557-0014, USA and ${ }^{3}$ Boston University School of Medicine, Department of Genetics and Genomics, Boston University, E632, Boston, MA 02118, USA

Email: Laurent G Deluc - delucl@unr.edu; Jérôme Grimplet - jerome.grimplet@sdstate.edu; Matthew D Wheatley - wheatle8@unr.nevada.edu; Richard L Tillett - tillett@unr.nevada.edu; David R Quilici - quilici@unr.edu; Craig Osborne - Vandal98@aol.com;

David A Schooley - schooley@unr.edu; Karen A Schlauch - schlauch@unr.edu; John C Cushman - jcushman@unr.edu;

Grant R Cramer* - cramer@unr.edu

* Corresponding author

Published: 22 November 2007

BMC Genomics 2007, 8:429 doi:10.1/86/147|-2164-8-429
Received: 14 May 2007

Accepted: 22 November 2007

This article is available from: http://www.biomedcentral.com/I47I-2/64/8/429

(c) 2007 Deluc et al; licensee BioMed Central Ltd.

This is an Open Access article distributed under the terms of the Creative Commons Attribution License (http://creativecommons.org/licenses/by/2.0), which permits unrestricted use, distribution, and reproduction in any medium, provided the original work is properly cited.

\begin{abstract}
Background: Grape berry development is a dynamic process that involves a complex series of molecular genetic and biochemical changes divided into three major phases. During initial berry growth (Phase I), berry size increases along a sigmoidal growth curve due to cell division and subsequent cell expansion, and organic acids (mainly malate and tartrate), tannins, and hydroxycinnamates accumulate to peak levels. The second major phase (Phase II) is defined as a lag phase in which cell expansion ceases and sugars begin to accumulate. Véraison (the onset of ripening) marks the beginning of the third major phase (Phase III) in which berries undergo a second period of sigmoidal growth due to additional mesocarp cell expansion, accumulation of anthocyanin pigments for berry color, accumulation of volatile compounds for aroma, softening, peak accumulation of sugars (mainly glucose and fructose), and a decline in organic acid accumulation. In order to understand the transcriptional network responsible for controlling berry development, mRNA expression profiling was conducted on berries of $V$. vinifera Cabernet Sauvignon using the Affymetrix GeneChip ${ }^{\circledR}$ Vitis oligonucleotide microarray ver. 1.0 spanning seven stages of berry development from small pea size berries ( $E-L$ stages 31 to 33 as defined by the modified E-L system), through véraison (E-L stages 34 and 35 ), to mature berries (E-L stages 36 and 38 ). Selected metabolites were profiled in parallel with mRNA expression profiling to understand the effect of transcriptional regulatory processes on specific metabolite production that ultimately influence the organoleptic properties of wine.
\end{abstract}

Results: Over the course of berry development whole fruit tissues were found to express an average of $74.5 \%$ of probes represented on the Vitis microarray, which has 14,470 Unigenes. Approximately $60 \%$ of the expressed transcripts were differentially expressed between at least two out of the seven stages of berry development ( $28 \%$ of transcripts, 4,15 I Unigenes, had pronounced ( $\geq 2$ fold) differences in mRNA expression) illustrating the dynamic nature of the developmental process. The subset of 4,15I Unigenes was split into twenty well-correlated expression profiles. 
Expression profile patterns included those with declining or increasing mRNA expression over the course of berry development as well as transient peak or trough patterns across various developmental stages as defined by the modified E-L system. These detailed surveys revealed the expression patterns for genes that play key functional roles in phytohormone biosynthesis and response, calcium sequestration, transport and signaling, cell wall metabolism mediating expansion, ripening, and softening, flavonoid metabolism and transport, organic and amino acid metabolism, hexose sugar and triose phosphate metabolism and transport, starch metabolism, photosynthesis, circadian cycles and pathogen resistance. In particular, mRNA expression patterns of transcription factors, abscisic acid (ABA) biosynthesis, and calcium signaling genes identified candidate factors likely to participate in the progression of key developmental events such as veraison and potential candidate genes associated with such processes as auxin partitioning within berry cells, aroma compound production, and pathway regulation and sequestration of flavonoid compounds. Finally, analysis of sugar metabolism gene expression patterns indicated the existence of an alternative pathway for glucose and triose phosphate production that is invoked from véraison to mature berries.

Conclusion: These results reveal the first high-resolution picture of the transcriptome dynamics that occur during seven stages of grape berry development. This work also establishes an extensive catalog of gene expression patterns for future investigations aimed at the dissection of the transcriptional regulatory hierarchies that govern berry development in a widely grown cultivar of wine grape. More importantly, this analysis identified a set of previously unknown genes potentially involved in critical steps associated with fruit development that can now be subjected to functional testing.

\section{Background}

Grapes have been cultivated and fermented into wine for more than 7,000 years. Worldwide, grapes are one of the most widely cultivated fruit crops, encompassing 7.4 million hectares of arable land in 2006 [1] and with 68.9 million metric tons produced in 2006, ranks second among bananas, oranges, and apples with 69.7, 63.8 and 62.1 million metric tons respectively, produced during this same period. However, because the majority of the grapes that are harvested are fermented into wine, the economic impact for this commodity is far greater than the value of the grapes. For example, wine sales from California alone in 2006 was at an all-time high and growing with approximately $\$ 18$ billion dollar in sales [2]. According to 2005 statistics, the California wine industry has a $\$ 52$ and $\$ 125$ billion economic impact on the state and U.S. economies, respectively [3].

In addition to their economic importance, consumption of grapes and wine has numerous nutritional and health benefits for humans $[4,5]$. For example, there are more than 200 polyphenolic compounds in red wines that are thought to act as antioxidants. In particular, one antioxidant compound, trans-resveratrol, has been shown to play a role in the prevention of heart disease (atherosclerosis) [6] and cancer [7]. Resveratrol slows the aging process in animals [8], acts as a signaling molecule in the brain [9], and down-regulates the expression of genes that are involved in cell cycle and cell proliferation in human prostate cells [10]. Therefore, for a variety of reasons, there is great interest in manipulating grape berry development and quality for both economic and health reasons.

In contrast to the well studied climacteric fruits such as tomato and apple, very little is known about the development and ripening processes of non-climacteric fruits such as grape or strawberry [11,12]. In 1992, Coombe, one of the leaders in the field, described our knowledge of grape berry development and the regulation of ripening as "embryonic [13]."

Grape berries, like other berry fruits, undergo a complex series of physical and biochemical changes during development, which can be divided into three major phases [13] with more detailed descriptive designations, known as the modified E-L system, being used to define more precise growth stages over the entire grapevine lifecycle [14]. During the initial stage of berry growth (Phase I) cell division is rapid and all cells are established in the developing fruit in the first two weeks after flowering followed by a subsequent sigmoidal increase in berry size over approximately 60 days due to cell expansion. Two important organic acids, tartrate and malate, are synthesized and reach maximal concentrations by the end of Phase I. Biosynthesis of tannins and hydroxycinnamates, which are major precursors for phenolic volatiles, also occurs, primarily during Phase I. Tannins are located primarily in the skin and seeds of the berry, and are perceived as astringent compounds important for color stability and the body of red wine. 
Phase II is characterized as a lag phase during which there is no increase in berry size. Biosynthetic processes are not well characterized for this stage, but it is known that sugar accumulation begins during this phase just prior to véraison (the onset of ripening) [13]. Véraison marks the start of Phase III of berry growth, which is characterized by the initiation of color development (anthocyanin accumulation in red grapes) and berry softening. Berry growth is sigmoidal during Phase III, as the berries double in size. At the onset of this stage, sugars (largely glucose and fructose) continue to accumulate, and organic acid concentrations decline. The acid:sugar balance at harvest is critical for high quality wines, as it affects important sensory attributes [15]. A large number of the flavor compounds and volatile aromas are synthesized at the end of Stage III. Many of these aromas are derived from terpenoids. However, the availability of seed tannins declines through oxidative processes during Phase III, causing the tannins to bind to the seed coat, reducing the astringent components within the berry. Skin tannins begin to interact and bind with anthocyanins and each other, increasing tannin polymer size and complexity.

Two major objectives of modern viticultural practices include the ability to produce a uniformly ripe crop and to harvest at optimal grape maturity. Large variations in ripening among berries within a cluster and within a vineyard make it difficult to determine when a crop is at its best possible ripeness. The start of véraison is recognized to be a critical determinant for berry harvest dates, yet little is known about what initiates this important stage. A more detailed understanding of the complex changes in gene expression that orchestrate berry developmental processes is needed.

Several mRNA expression-profiling studies have been completed for Vitis berries. Differential screening of cDNA libraries from (Vitis vinifera cv. Shiraz) and northern blot analysis revealed that large differences in gene expression occur during berry ripening and led to the isolation of a large number of grape ripening-induced protein (GRIP) genes [16]. Monitoring of gene expression profiles in flowers and across six time points during grape (Vitis vinifera $\mathrm{cv}$. Shiraz) berry skin development to 13 weeks postflowering resolved four sets of genes with distinctive and similar expression patterns using spotted cDNA microarrays containing 4,608 elements [17]. mRNA expression was also studied across nine stages of wildtype cv. Shiraz berry development (green "pea" to overripe) [18] and in a fleshless berry mutant $\mathrm{cv}$. Ugni Blanc using oligonucleotide microarrays containing 3,200 elements [19]. Differences in transcript expression profiles in the skin of ripening fruit (12 to 13 weeks after flowering) of seven different cultivars were also examined using a 9,200 feature cDNA microarray [20]. In this study, we conducted
mRNA expression profiling on one of the widely grown varieties of $V$. vinifera (cv Cabernet Sauvignon) using the Vitis Affymetrix GeneChip ${ }^{\circledast}$ oligonucleotide microarray ver. 1.0, which contains 14,470 Unigenes, over seven temporal stages (green "pea" to ripe) of berry development. We also correlated specific transcript profiles with specific metabolite profiles to gain deeper insights into discrete aspects of grape berry developmental dynamics.

\section{Results and discussion Grape berry development}

Vitis vinifera cv. Cabernet Sauvignon grapes were harvested on a weekly basis over the course of berry development from the Shenandoah Vineyard, Plymouth, California during the summer of 2004. Samples corresponding to stages 31 to 38 of the modified E-L system [14] were measured for berry diameter, ${ }^{\circ}$ Brix (an approximate measure of the mass ratio of dissolved solids, mostly sucrose, to water in fruit juices) and titratable acidity (Figure 1). Berry diameter increased over time with a classical double sigmoid pattern (Figure 1A). Average berry diameter increased during the first 7 weeks of development (E-L stage 31), followed by a cessation of berry expansion at 7 to 8 weeks post-anthesis (E-L stages 32 to $34)$, and then the increase in berry diameter resumed until maturity (E-L stages 35 to 38). ${ }^{\circ}$ Brix increased 6 weeks post-anthesis to a peak value of $22^{\circ}$ Brix at 16 weeks postanthesis (Figure 1B). In contrast, titratable acidity $(\mathrm{g} / \mathrm{L})$, which reflects acid accumulation (mainly tartaric and malic acid), increased steadily up to 8 weeks post-anthesis and then sharply declined at the start of veraison between E-L stages 34 and 35 reaching approximately $7 \mathrm{~g} / \mathrm{L}$ of titratable acids at harvest (Figure 1C).

\section{Microarray analysis}

The mRNA expression profiles of seven time points spanning E-L stages 31 to 38 as indicated in Figure 1 were compared using the Affymetrix GeneChip ${ }^{\circledR}$ Vitis genome array ver. 1.0. Testing was performed using biological triplicates for each time point. Multiple time points within Stage II (E-L stages 32 to 35 ) were sampled due to the large number of biochemical changes expected to occur around véraison that affect berry ripening and fruit quality. A visual inspection of the distributions of raw perfect match (PM) probe-level intensities for all 21 arrays showed that the pre-processed and normalized PM intensities using Robust Multi-Array Average (RMA) [21] were consistent across all arrays. Digestion curves describing trends in RNA degradation between the $5^{\prime}$ end and the 3 ' end in each probe set were examined and all 21 proved very similar [Additional File 1A,B]. Correlations among biological replicates were good: Spearman coefficients ranged from 0.977 to 0.997; Pearson coefficients ranged between 0.977 and 0.996 . 


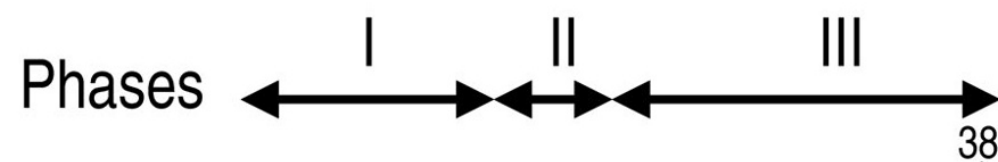

A
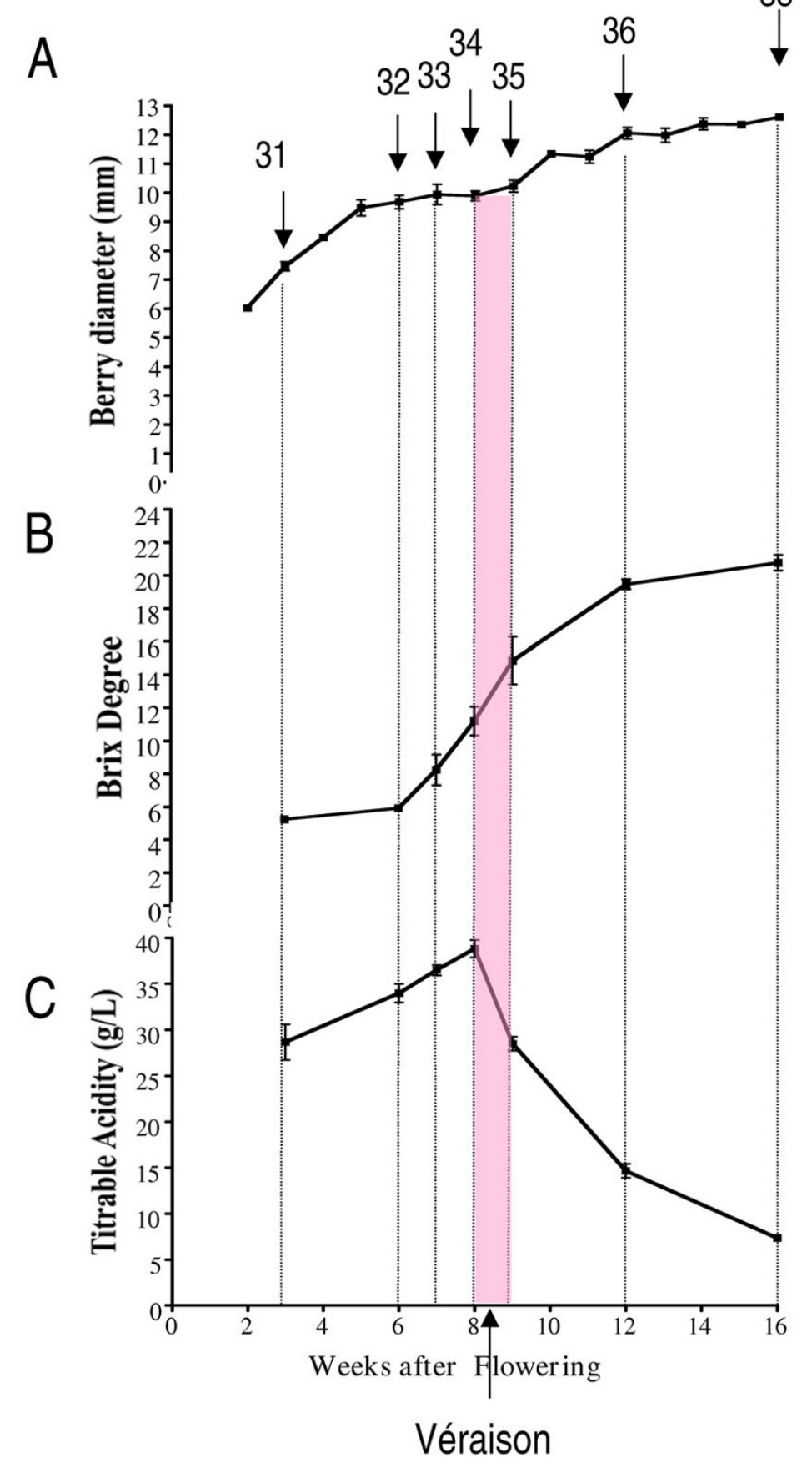

Figure I

Physiological data at different stages of berry development. Changes in physiological parameters measured during the major phases (I to III) of berry development and ripening of Cabernet Sauvignon grape berries. A, Berry Diameter $(n=6)$; B, Brix degree $\left({ }^{\circ}\right)$ or total soluble solids in the berry juice $(n=6)$; C, Titratable Acidity $(g / L)(n=6)$. The stage at which veraison occurs is indicated in pink. Numbers with arrows point to the individual developmental stages defined by the E-L system Coombe [14] used for transcriptome profiling. 
From the Vitis 16,602 probesets represented on the array [Additional File 1C], an overall mean call rate of $74.5 \%$ per array (range $73.5 \%$ to $76.2 \%$ ) was obtained. Data from the 12,596 probe sets that were found to be present in at least two out of the three biological triplicates were retained for further analyses. After performing an ANOVA and a multiple testing correction (Benjamini and Hochberg) [22], we found that 10,068 probesets $(60.6 \%)$ were differentially expressed $(\mathrm{p} \leq 0.05)$ between two or more EL stages of berry development [Additional File 2: Table 1]. Because one Unigene can be related to several probesets, the number of Unigenes decreased to 9,143 Unigenes [Additional File 2: Table 2]. These probesets will be hereby referred to as those passing the ANOVA filter. From this set of genes, we extracted a subset of 4,510 probesets that displayed a two-fold or greater change in steady-state transcript abundance over the course of development (i.e., across any two of the seven developmental stages) [Additional File 2: Table 3] representing 4,151 Unigenes $(28.3 \%)$ in the DFCI Grape Gene Index database VvGI5 [23]. We refer to this subset of genes as the two-fold ratio (TFR) set [Additional File 2: Table 4].

Principal component analysis (PCA), was used to simplify and define associations between different developmental stages within the global transcriptomic data (Additional File 3). Two principal components explaining $97.4 \%$ of the overall variance of transcription profiles $(86.8 \%$ and $7.6 \%$ for axes 1 and 2, respectively) allowed us to clearly differentiate E-L stages 31 and 35 from the other developmental stages analyzed (Additional File 3 ). It was not possible to clearly separate E-L stages 32 to 34 or 36 to 38 indicating that the transcription patterns occurring at these stages were similar to one another. However, stage 35 , which corresponds to early post-véraison, could be distinguished suggesting that transcription patterns at this point in berry ripening are unique to this critical stage in berry development. Further analysis using a third axis explaining $2.7 \%$ of the overall variance, confirmed the previous results and slightly improved the resolution among stages 31,35 , and 36 to 38 .

\section{Clustering of significant genes}

We used the Pavlidis Template Matching (PTM) algorithm [24], to divide the 4,151 TFR Unigenes into twenty gene groups or clusters. Specifically, twenty gene profiles of interest were selected [Additional File 4] to reflect major transcriptional patterns of development across E-L stages 31 to 38 (Figure 2). The PTM algorithm then classified the gene profiles into twenty groups via measurements of Pearson correlation: a correlation coefficient of greater than 0.75 was used to determine cluster membership. Six profiles showed a steady decline (profile groups 1 to 3 ) or increase (profile groups 9 to 11 ) in steady-state transcript abundance over time with distinctly different slopes.
These six profile groups encompassed $63 \%$ of the Unigenes with a majority expressed in profiles 2 and 3 $(31.9 \%)$ and profiles 9 and 11 (28\%; Figure 2). Eight profiles had transient peak increases (profile groups 4 to 8 ) or decreases (profile groups 12 to 16) in transcript abundance at each of E-L stages 32 to 36. These transient profiles accounted for $22 \%$ of the Unigenes. A majority $(68.2 \%)$ of these transiently expressed genes (profile groups 4 to 8 and 12 to 16) exhibited increased transcript abundance with the highest proportion within profile group 16 (E-L stage 36), followed closely by profile group 15 (E-L stage 35 around véraison), and profile group 12 (E-L stage 32) (Figure 2). Interestingly, genes with transient decreases early in berry development (profile groups 4 and 5) also exhibited large increases in transcript abundance during the later stages (E-L stages 36 to 38 ). The last four profiles (profile groups 17-20) were selected as having two peaks of expression between E-L stages 32 and 36 (Figure 2). Approximately $4.3 \%$ of transcripts had such "up and down" expression patterns (profile groups 17-20). Finally, Unigenes that did not match one of these profiles were grouped into a $21^{\text {st }}$ cluster (Figure 2), accounting for $11 \%$ of the total transcripts considered (profile group 21). Taken together, this analysis revealed that berry development is not only a progressive process, wherein the majority of genes exhibit a steady increase or decrease in expression across all stages of development (profile groups 1 to 3 and 9 to 11), but also a dynamic process, wherein a large number of genes exhibit large, transient changes in transcript abundance at specific times of development. Most notably, the last phase of berry development (Phase III, profile groups 14, 15 and 16) was the time when the largest number of genes (380 transcripts or $9.1 \%$ ) exhibited transient increases in steadystate transcript abundance.

\section{Functional categorization of Unigenes across different stages of development}

Functional categories were assigned to Unigenes with twofold or greater changes in steady-state transcript abundance over the course of the seven developmental stages using the Munich Information Center for Protein Sequences (MIPS, ver. 2.0) catalog with annotations of the top Arabidopsis BLAST hits [25]. Because we detected some errors in the functional annotation for some Unigenes, functional categorization of each Unigene were verified manually and corrected if necessary. Corrections were only performed for the 4,151 Unigenes that displayed a two-fold or greater change in expression [See Additional File 2: Table 4]. Functional annotations could be assigned to approximately $64 \%$ of transcripts (Figure $3 \mathrm{~A}$ ). An additional $23 \%$ of Unigenes had matches to genes with unknown functions or unclear classifications (unclassified), and 13\% did not have a BLAST hit (no hit) in public, non-redundant (NR) databases. The relative 
(V)

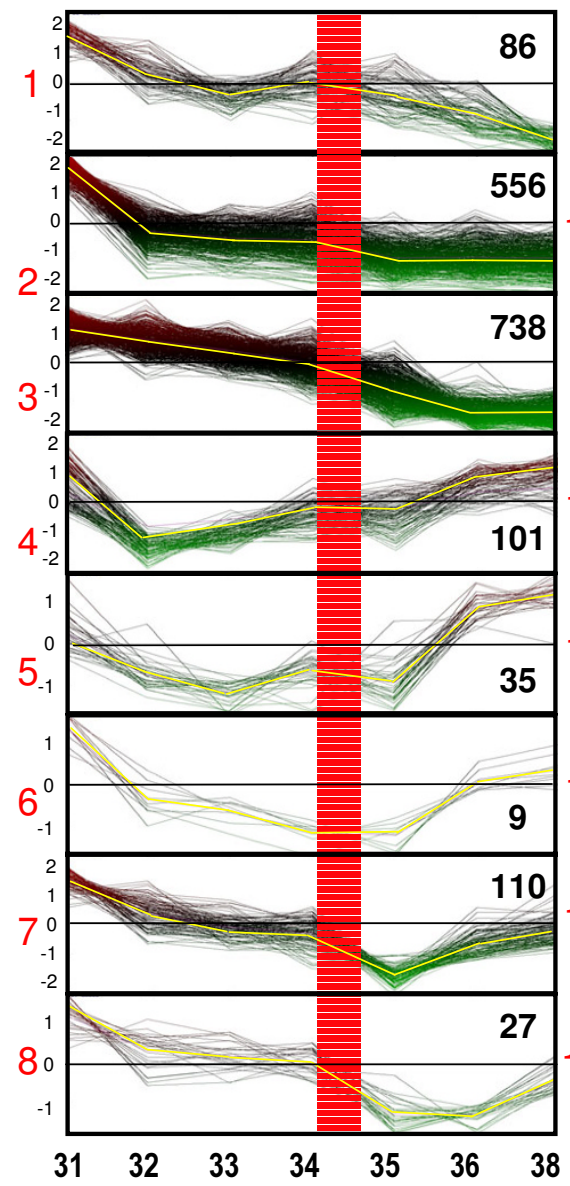

(V)

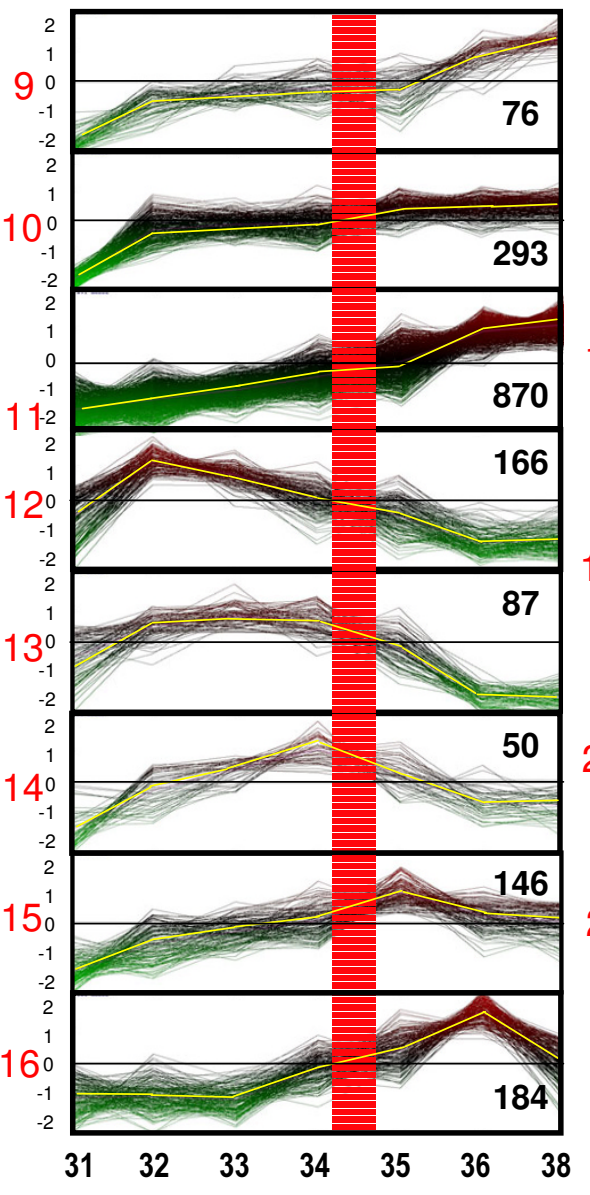

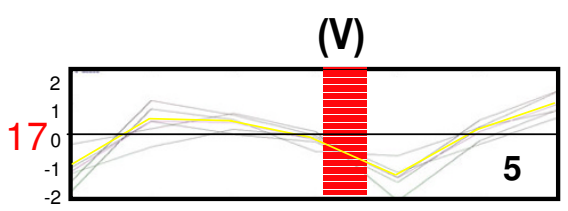
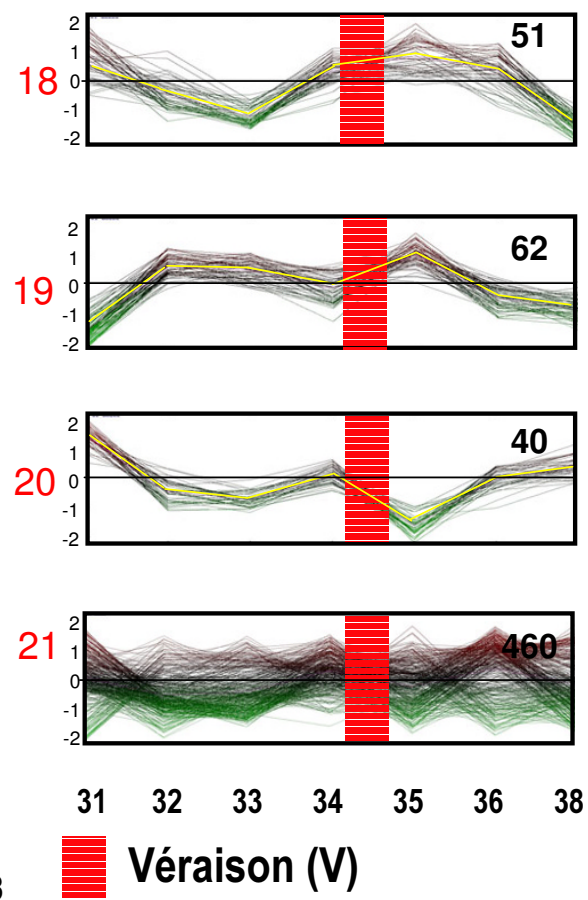

Figure 2

Twenty-one profiles of steady-state transcripts exhibiting a two-fold or greater expression across berry development. Profiles are plotted as RMA data values plotted on the $\log _{2}$ scale centered by the mean of all values (Stage $3 \mathrm{I}$ to stage 38). E-L Stages are indicated along the $X$-axis. Profiles numbers are indicated with red numbers with the number of transcripts within each profile indicated with black numbers: Véraison $(\mathrm{V})$ is indicated with a pink stripe. The gradient red to green coloration of individual gene plots indicates values above or below the mean of the cluster, respectively. The cluster template profile is designated by a yellow line.

distribution of Unigenes within each of nineteen functional categories was determined (Figure 3B). To facilitate a functional comparison of the three major stages of berry development, Unigenes from each of the profile groups were regrouped into the three major developmental phases to reflect the greatest degree of transcript abundance changes at each phase: Phase I (profiles 1, 2, and 3), Phase II (profiles 4, 5, 6, 12, 13, and 14), and Phase III (profiles 7, 8, 9, 10, 11, 15, and 16). Statistically significant differences in the distribution of genes within functional categories amongst these developmental stages were observed (Figure 3B; see Additional File 5: Tables 1 and 2). Functional categories that had a large number of transcripts in Phase I followed by a decrease in Phase III included biogenesis of cellular component (42), transport regulation (20), energy (2), and metabolism (1). This is consistent with the developmental aspects of this phase, which are characterized by cell division and expansion, which require a high level of metabolic activity. The process of cell division requires large quantities of structural materials and consumes energy, while cell expansion requires large quantities of solutes and water.

The opposite trend of increasing transcript abundance from Phase I to Phase III was observed for functional groups that included transcription (11), protein synthesis 


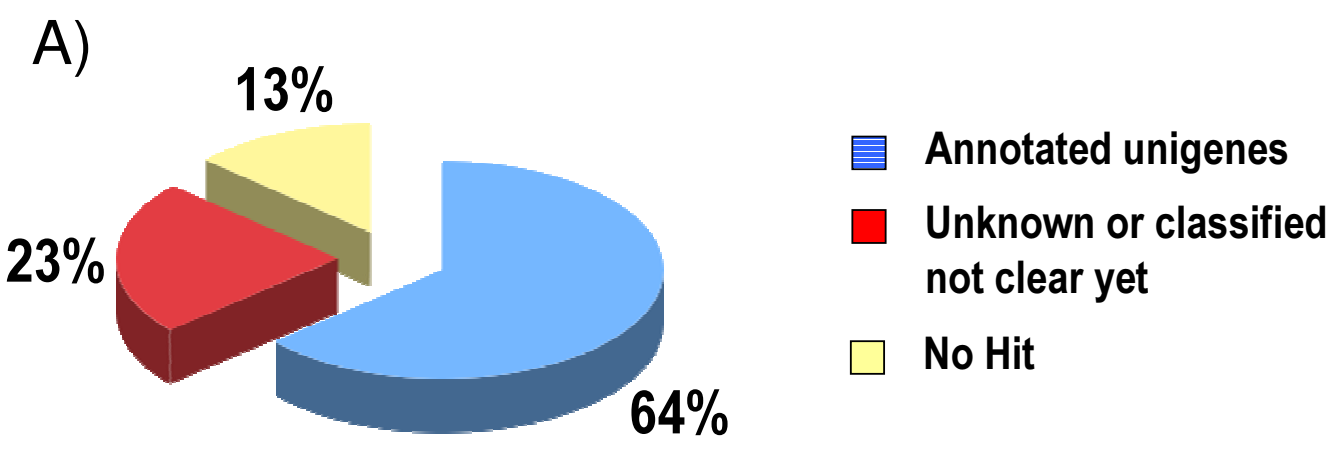

B)

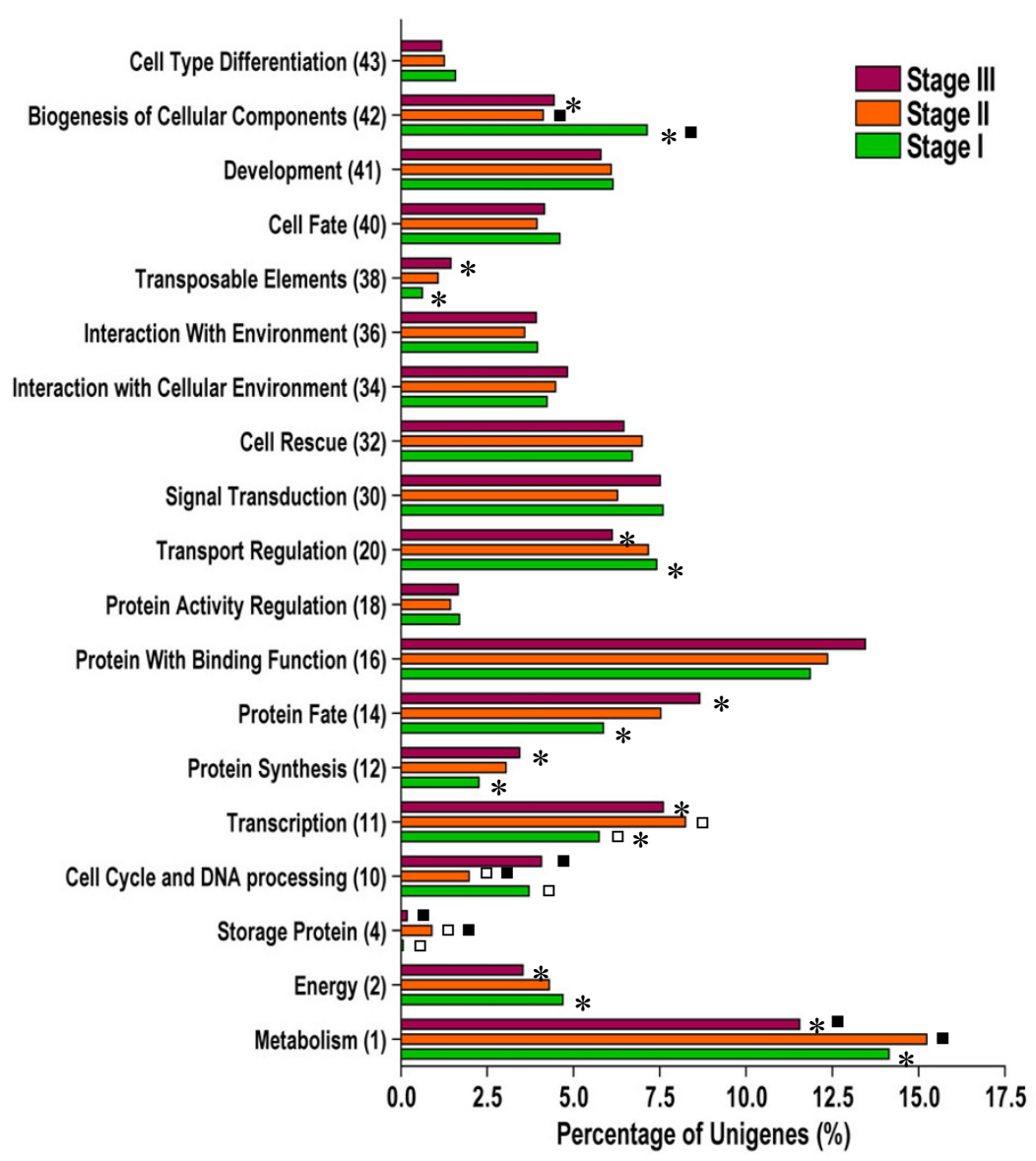

\section{Figure 3}

Functional analyses of steady-state transcripts with a two-fold or greater change in abundance over the course of berry development. A) Percentage of annotated unigenes with a two-fold or greater change in transcript abundance. B) Distribution of Unigenes according to their MIPS functional categories (MIPS 2.0) within the three main phases of berry development. Phase I (E-L stage 3I), herbaceous phase; Phase II (E-L stages 32 to 34), lag phase; Phase III (E-L stages 35 to 38 ), ripening phase. Statistically significant differences between Phase I against II are indicated with white squares. Statistically significant differences between Phase II against III are indicated with black squares. Statistically significant differences between Phase I against III are indicated with asterisks. Percentages are based upon the number of Unigenes in each set. Numbers in parentheses following category names indicates the MIPS number for each category. 
(12), protein fate (14), protein with binding function (16), and to a lesser extent with interaction with cellular environment (34). These trends served to further indicate the complexity of the transcriptional, translational, and interaction-based regulatory processes necessary for berry development.

\section{Exemplar Unigenes associated with important molecular events of berry development}

In order to identify genes with potentially important roles in specific stages of berry development, transcripts with a dynamic pattern were identified from within the first 20 PTM algorithm-defined profile groups. The transcript profiles were examined in further detail (Figure 4).
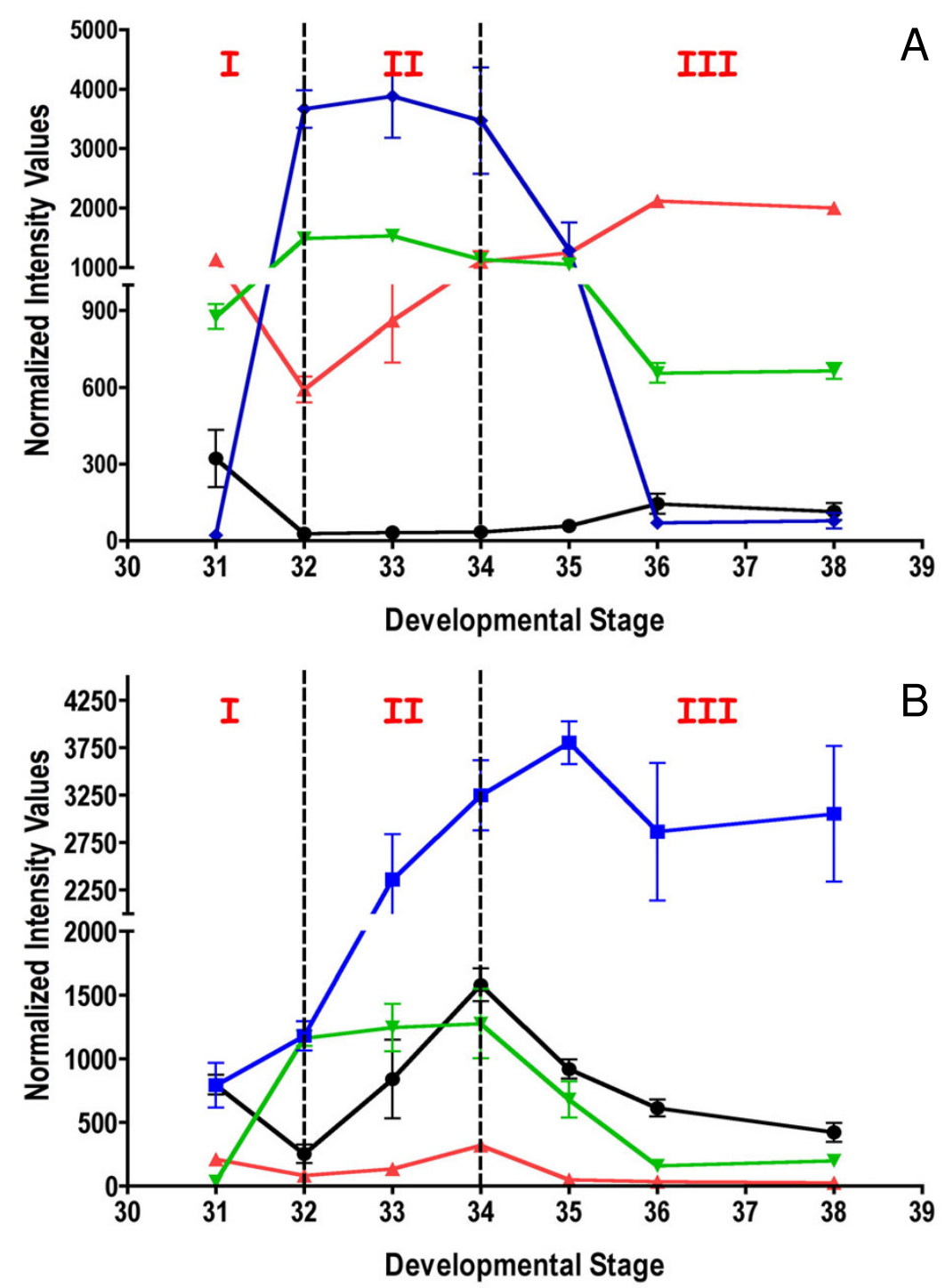

\section{Figure 4}

Transcripts displaying transient expression patterns. Each value plotted is the mean normalized intensity values obtained for the three biological replicates. The three key phases of the berry development (I, II, III) were applied as reference. A) Black solid round (16188|4_at, NP864096)-ornithine decarboxylase, red solid triangle (1616399_s_at, CB005833)-arginine decarboxylase, green solid triangle (16 | I257_a_at, TC5 I832)-L-asparaginase, blue solid diamond (16 I8848_at, TC52577)xyloglucan endotransglycosylase transferase. B) Black solid round (1608074_s_at, TC62965)- $\alpha$-expansin, red solid triangle (1608191_at, TC64448) $\alpha$-expansin, green solid triangle (1613161_at, TC69794)-limonene cyclase, blue solid diamond (1618595_at, TC5384I)-(-)-isopiperitenol dehydrogenase. 
Polyamines (PAs) are a class of compounds that have plant growth regulator activity. Their roles in cell division [26] and fruit set [27] have been widely investigated. Free, conjugated and wall-bound forms of polyamines accumulate mostly at anthesis before decreasing at fruit set in grapes [28]. Two transcripts were detected that belong to profile 4, which are strongly down-regulated at E-L stage 32 (1618814_at, 1616399_s_at; AY174164, TC68466). Both are related to ornithine decarboxylase and arginine decarboxylase, which are involved in polyamine metabolism [29]. These two genes located at the start of the PA pathway might play a role in providing precursors that would be used during Phase I of berry development.

In higher plants, the catabolism of asparagine (Asn) occurs by two routes. The first pathway involves the hydrolysis of Asn, releasing ammonia and aspartate by asparaginase activity. L-asparaginase is one of the enzymes for Asn utilization by plants that plays an important role in the nitrogen metabolism of developing plant tissues [30]. One Unigene encoding L-asparaginase (1611257_a_at; TC51832) displayed a specific peak during E-L stages 32 (Figure 4A). This last result indicates that this enzyme could play a role during the first phase of berry development as a provider of ammonia for de novo protein synthesis in grape. This result is also supported by the significant transcript abundance of Unigenes encoding glutamate dehydrogenase or glutamine synthetase (data not shown, see Additional File 2: Table 4, 1607579_at, 1613697_at, 1609819_s_at) during the first phase of berry development. These enzymes participate in nitrogen assimilation in plants [31].

In grape berries, fruit softening occurs during Phase III and is largely affected by cell-wall loosening [32] and turgor [33]. Xyloglucans account for about $10 \%$ of the cell wall composition in berries [32]. In fruit, xyloglucan depolymerization is associated with fruit softening [34]. Xyloglucan endotransglycosylases, which hydrolyze and transglycosylate xyloglucans, were encoded by multiple isogenes, the majority of which were expressed highly during Phase I in berry development (E-L stage 31), but then declined (data not shown; see Table 1). One Unigene (1618848_at; TC52577), however, which is a xyloglucan endotransglucosylase/hydrolase, displayed a 185-fold increase in expression during Phase II, peaking at E-L stage 33 (Figure 4A). This xyloglucan endotransglucosylase Unigene is closely related to a xyloglucan endotransglucosylase/hydrolase (SIXTH5) that can act reversibly. It has been characterized recently as a tomato xyloglucan depolymerase in vitro in the presence of xyloglucan oligosaccharides (XGOs) [35].

Expansins play important roles in cell wall loosening via non-enzymatic mechanisms and are involved in cell expansion [36]. Most expansin genes displayed steadily increasing or decreasing patterns during berry development (see Table 1). Others showed peak expression around E-L stage 34 ( $\alpha$-expansin, 1608074_at, TC62965; $\alpha$-expansin, 1608191_at, TC64448; Figure 4B). An expansin gene from strawberry, FaExp4, displays exactly the same peak transient expression pattern as these latter two genes at a comparable ripening stage as grape berries, called the White stage in strawberry fruits, just before red fruit color development [37]. Thus, these expansins in grape berry may be required during the Phase III of grape berry development, when the second phase of cell expansion occurs.

Terpenes, which are precursors for important aroma compounds [38], accumulate at véraison [39,40]. One Unigene encoding a limonene cyclase (1613161_at; TC69794; Figure 4B), which is in the monoterpene pathway, is involved in the conversion of geranyl diphosphate into limonene [41]. Limonene and some of its derived compounds such as menthol or 1,8 cineol are intimately associated with the "eucalyptus fragrance" of red wine [42]. Accumulation of 1,8-cineole in wines is derived from precursors in grape, like limonene. The strong induction of our Unigene related to limonene cyclase ( $\sim 40$ fold from E-L stages 32 to 34) correlates well with the beginning of accumulation of 1,8-cineole in red grape samples [43]. One Unigene (1618595_at, TC53841; Figure 4B) belonging to profile 15 and encoding alcohol dehydrogenase exhibited strong homology with an (-) isopiperitenol dehydrogenase, which is involved in the same monoterpene pathway [44]. This transcript abundance of this Unigene is correlated to the expression of the limonene cyclase previously discussed above indicating a possible activation of these enzymes in the same metabolic pathway [44]

\section{Phytohormone biosynthesis and responses}

A number of plant growth regulators including abscisic acid (ABA), auxin (indole-3-acetic acid [IAA], brassinosteroids (BR), ethylene, and gibberellic acid (GA) have been implicated in the control of berry development and ripening. Therefore, steady-state transcript accumulation patterns of Unigenes with functions related to hormone biosynthesis and response were tracked over the course of berry development (Figure 5, Table 2).

\section{Abscisic acid}

ABA amounts in berries decrease after anthesis, but then increase significantly at véraison [45]. External applications of ABA to ripening fruit can accelerate berry development (see [13] and references therein). The transcript abundance of 9-cis-epoxycarotenoid dioxygenase (NCED), which encodes the rate limiting step in ABA biosynthesis, increased during the lag phase and peaked at 
Table I: Transcripts (TFR pool) related cell wall metabolism categorized by the first hit in the MIPS2 catalog

\begin{tabular}{|c|c|c|c|c|c|c|c|}
\hline Probeset ID & $\begin{array}{l}\text { GenBank } \\
\text { Annotation }\end{array}$ & VvGI5 & UniProt ID & $\begin{array}{l}\text { Gene Name } \\
\text { Description }\end{array}$ & Function & Profile & Fold Change \\
\hline |62279|_at & CB973455 & TC56II4 & Q6J8X2 & Cellulose synthase & Cell Wall Biosynthesis & 2 & 112.68 \\
\hline 1619280_at & CF21I860 & TC59569 & Q6J8W9 & Cellulose synthase & Cell Wall Biosynthesis & 2 & 87.25 \\
\hline 1613018_at & CB97III7 & TC6I56I & Q85IL8 & Cellulose synthase & Cell Wall Biosynthesis & 2 & 6.92 \\
\hline 1606646_at & CA812296 & TC56773 & Q6XZC2 & Cellulose synthase & Cell Wall Biosynthesis & 2 & 4.34 \\
\hline 1615577_at & CB340193 & TC52068 & Q6XP46 & Cellulose synthase & Cell Wall Biosynthesis & 3 & 3.5 \\
\hline 1607069_at & CB982496 & TC5345I & Q45KQ0 & Cellulose synthase & Cell Wall Biosynthesis & 10 & 3.18 \\
\hline 1611149_at & BM437543 & TC5609I & Q3Y6VI & Cellulose synthase & Cell Wall Biosynthesis & 21 & 2.85 \\
\hline 1612999_at & CF5I3786 & - & 080890 & Cellulose synthase & Cell Wall Biosynthesis & 7 & 2.76 \\
\hline 1620206_at & CF5I55I9 & TC66132 & Q6FD0 & $\beta$ I,4-Mannan synthase & Cell Wall Biosynthesis & 15 & 2.69 \\
\hline 1616808_at & CF207742 & TC57597 & Q45KQ0 & Cellulose synthase & Cell Wall Biosynthesis & 21 & 2.21 \\
\hline 1619938_at & CF5I4664 & TC63356 & Q6YBV2 & Cellulose synthase & Cell Wall Biosynthesis & 3 & 2.19 \\
\hline 1620840_at & CB968965 & TC53122 & Q4F8K2 & $\alpha$-expansin & Cell Wall Expansion & 2 & 20.26 \\
\hline 1619010_s_at & BQ794765 & TC54832 & Q84US9 & Expansin & Cell Wall Expansion & 10 & 18.54 \\
\hline |60819|_at & CD79883I & TC64448 & Q49QW6 & Expansin & Cell Wall Expansion & 21 & 13.18 \\
\hline 1612253_at & CB970527 & TC62108 & Q6RX68 & Expansin & Cell Wall Expansion & 3 & 9.23 \\
\hline 1608074_s_at & CF2I5793 & TC62965 & Q84UT0 & Expansin & Cell Wall Expansion & 21 & 6.28 \\
\hline 1610418_at & BQ798078 & TC67284 & Q8GZD3 & Expansin & Cell Wall Expansion & 10 & 4.9 \\
\hline 1613527_at & CB978490 & TC53065 & Q6T5H5 & Expansin & Cell Wall Expansion & 15 & 4.6 \\
\hline 1618121 at & CF2I369I & - & Q9LUII & Extensin & Cell Wall Expansion & 2 & 4.1 \\
\hline 1608504_at & BQ79723I & TC52168 & Q6K4C6 & Expansin & Cell Wall Expansion & 4 & 2.56 \\
\hline 1612154_at & CB970048 & TC6I667 & O50044 & KDO 8-P synthase & Cell Wall Expansion & 3 & 2.31 \\
\hline 160965I_at & CF404678 & TC55463 & Q9LJX2 & Pectinesterase inhibitor & Cell Wall Modification & 2 & 690.87 \\
\hline 1618848_at & СВ977336 & TC52577 & Q9ZRVI & $\begin{array}{l}\text { Xyloglucan } \\
\text { endotransglycosylase I }\end{array}$ & Cell Wall Modification & 13 & 184.42 \\
\hline 1622288_at & CB974798 & TC59058 & Q9M660 & Cell-Wall P4 & Cell Wall Modification & 2 & 124.19 \\
\hline 1617556_s_at & BQ797260 & TC67257 & Q9M4II & $\begin{array}{l}\text { Proline-rich cell wall } \\
\text { protein }\end{array}$ & Cell Wall Modification & 10 & 105.38 \\
\hline 1619762_at & CF2I4586 & TC67718 & Q7Y250 & Arabinogalactan protein & Cell Wall Modification & 2 & 61.79 \\
\hline 162020I_at & CB972625 & TC70982 & Q53WM8 & Pectinesterase & Cell Wall Modification & 2 & 42.57 \\
\hline 1619519_at & CB97I445 & TC65487 & Q7Y250 & Arabinogalactan protein & Cell Wall Modification & 2 & 39.53 \\
\hline 1616045_a_at & AJ237983 & - & Q9M410 & $\begin{array}{l}\text { Proline-rich cell wall } \\
\text { protein }\end{array}$ & Cell Wall Modification & 11 & 38.03 \\
\hline 1617023_at & CF210510 & TC53552 & FLAI & Arabinogalactan protein & Cell Wall Modification & 3 & 37.53 \\
\hline 1611601_at & CB977009 & TC57247 & Q6ZDX2 & Pectinesterase & Cell Wall Modification & 2 & 34.83 \\
\hline 1619613_at & CD801720 & TC68597 & Q9SAP3 & Proline-rich protein & Cell Wall Modification & 2 & 34.56 \\
\hline 1616528_s_at & CD80I342 & TC55I88 & QISAY6 & Proline-rich protein & Cell Wall Modification & 2 & 33.73 \\
\hline 1621880_s_at & CKI38206 & TC66098 & Q8VZG5 & $\beta$-xylosidase & Cell Wall Modification & 3 & 31.38 \\
\hline 1608727_at & CB973483 & TC56396 & Q9LZX4 & $\begin{array}{l}\text { Fasciclin arabinogalactan } \\
\text { protein } 10\end{array}$ & Cell Wall Modification & 3 & 30.04 \\
\hline 1615533_s_at & CF4I5374 & TC5I824 & Q7Y252 & $\begin{array}{l}\text { Endo-xyloglucan } \\
\text { transferase }\end{array}$ & Cell Wall Modification & 3 & 27.95 \\
\hline 162248I_x_at & CF56892I & TC67I50 & Q39763 & Proline-rich protein & Cell Wall Modification & 1 & 27.63 \\
\hline 1614426_at & CD80III6 & TC64I84 & Q4F8J3 & $\begin{array}{l}\text { Xyloglucan } \\
\text { endotransglycosylase }\end{array}$ & Cell Wall Modification & 3 & 25.94 \\
\hline 1619522_at & AY04323I & TC56838 & Q94BI7 & $\beta$-galactosidase & Cell Wall Modification & 3 & 24.53 \\
\hline 1622292_at & CF403386 & TC69174 & Q949ZI & polygalacturonase & Cell Wall Modification & 2 & 24.24 \\
\hline 1622295_at & CF215662 & TC6854I & Q5CCP8 & $\beta$-galactosidase & Cell Wall Modification & 3 & 24.1 \\
\hline |62|477_s_at & CF2I5974 & TC67884 & Q9LYT5 & Pectinesterase & Cell Wall Modification & 1 & 23.62 \\
\hline |622121_at & BQ799039 & TC58094 & Q4F8J0 & Cellulase & Cell Wall Modification & 3 & 22.85 \\
\hline 161520I_at & CF512517 & TC63907 & Q96232 & Proline-rich-like protein & Cell Wall Modification & 3 & 22.42 \\
\hline 1618657_at & CF2111626 & TC56055 & Q84LI7 & Exopolygalacturonase & Cell Wall Modification & 2 & 20.99 \\
\hline 1616158_at & CD80I7I7 & TC53176 & Q4JLV6 & Pectate lyase & Cell Wall Modification & 21 & 20.15 \\
\hline 1612239_at & CF610039 & TC5542I & Q8VZG5 & $\beta$-xylosidase & Cell Wall Modification & 2 & 19.96 \\
\hline 1620140_at & CF208989 & TC53499 & Q40161 & Polygalacturonase & Cell Wall Modification & 2 & 19.6 \\
\hline 1611747_at & CF608890 & TC65II3 & Q7XAS3 & $\beta$-D-glucosidase & Cell Wall Modification & 3 & 17.94 \\
\hline 1609909_s_at & CF206328 & TC64I84 & Q4F8J3 & $\begin{array}{l}\text { Xyloglucan } \\
\text { Endotransglycosylase }\end{array}$ & Cell Wall Modification & 3 & 15.14 \\
\hline |6083|3_at & CF209I44 & TC52275 & Q76MS4 & $\beta$-xylosidase & Cell Wall Modification & 2 & $|4.4|$ \\
\hline 1615574_at & CB977067 & TC56317 & Q9M5J0 & Pectinesterase & Cell Wall Modification & I & 14.03 \\
\hline
\end{tabular}


Table I: Transcripts (TFR pool) related cell wall metabolism categorized by the first hit in the MIPS2 catalog (Continued)

\begin{tabular}{|c|c|c|c|c|c|c|c|}
\hline 16196/2_at & CF2II6II & TC674I4 & Q94KD8 & $\beta$-xylosidase & Cell Wall Modification & 2 & 13.83 \\
\hline 1610073_at & CF206I57 & TC5I796 & Q8S902 & $\begin{array}{l}\text { Xyloglucan } \\
\text { Endotransglycosylase }\end{array}$ & Cell Wall Modification & 3 & 13.77 \\
\hline |62|25I_s_at & BQ795002 & TC69305 & Q8W3L8 & $\begin{array}{l}\text { Xyloglucan } \\
\text { Endotransglycosylase } 2\end{array}$ & Cell Wall Modification & 10 & 13.64 \\
\hline 1622735_s_at & CB340I22 & TC5I796 & Q84JX3 & $\begin{array}{l}\text { Xyloglucan } \\
\text { Endotransglycosylase }\end{array}$ & Cell Wall Modification & 3 & 13.47 \\
\hline 1613844_at & CF404099 & TC54968 & Q9LUG8 & $\begin{array}{l}\text { Endo-I,3-I,4- } \beta-D- \\
\text { glucanase }\end{array}$ & Cell Wall Modification & 3 & 13.27 \\
\hline 1617755_at & $C F 213513$ & TC52924 & Q8GSQ4 & $\begin{array}{l}\text { Pectin- } \\
\text { glucuronyltransferase }\end{array}$ & Cell Wall Modification & 2 & 12.86 \\
\hline 1615746_at & CB970034 & TC53433 & Q9FXI9 & Endo-I,4- $\beta$-glucanase & Cell Wall Modification & 3 & 11.99 \\
\hline 1617785_at & CD800I22 & TC5468I & Q9LW90 & Pectinesterase & Cell Wall Modification & 3 & 11.97 \\
\hline 1607374_at & CF404I62 & TC69448 & Q7XAS3 & $\beta$-D-glucosidase & Cell Wall Modification & 3 & 11.77 \\
\hline 1610311_at & CF373485 & TC52429 & Q4I725 & Arabinogalactan protein & Cell Wall Modification & 3 & 10.99 \\
\hline 1620096_at & CF37284I & TC57673 & Q4F986 & $\begin{array}{l}\text { Xyloglucan } \\
\text { endotransglycosylase }\end{array}$ & Cell Wall Modification & 2 & 10.81 \\
\hline 1616093_at & CF404665 & TC69415 & Q7XA92 & Pectinesterase & Cell Wall Modification & 3 & 10.36 \\
\hline 1613467_at & CF2II2805 & TC54247 & Q9FSW6 & Arabinogalactan protein & Cell Wall Modification & 15 & 10.08 \\
\hline 1617875_at & CB97I 740 & TC6I493 & O04477 & $\begin{array}{l}\beta-\mathrm{N}- \\
\text { acetylhexosaminidase }\end{array}$ & Cell Wall Modification & 3 & 9.73 \\
\hline 1614803_at & AY0464I6 & TC70I08 & Q8LGR6 & Proline-rich protein & Cell Wall Modification & 3 & 9.22 \\
\hline 1616822_at & AF220196 & TC70108 & Q8LGR6 & Proline rich protein & Cell Wall Modification & 3 & 9.04 \\
\hline 1610756_at & CF604824 & TC55088 & Q9LT39 & $\begin{array}{l}\text { Polygalacturonase } \\
\text { inhibitor }\end{array}$ & Cell Wall Modification & 1 & 8.2 \\
\hline |62259|_at & CB98II29 & TC70200 & Q9FHN6 & Pectinesterase & Cell Wall Modification & 2 & 8.06 \\
\hline 1612672_at & CF2I5975 & TC62593 & Q9SEE7 & Pectinesterase & Cell Wall Modification & 2 & 7.61 \\
\hline 1616522_at & CF403905 & TC55346 & Q9LEB0 & Pectinesterase & Cell Wall Modification & 2 & 7.51 \\
\hline 1615198_at & CF209943 & TC65883 & Q9LEC9 & $\beta$-xylosidase & Cell Wall Modification & 3 & 7.48 \\
\hline 1608756_at & BQ798436 & TC59719 & Q84LI7 & Polygalacturonase & Cell Wall Modification & 2 & 7.15 \\
\hline 1609790_at & CF207994 & TC55069 & Q4F8J3 & $\begin{array}{l}\text { Xyloglucan } \\
\text { endotransglycosylase }\end{array}$ & Cell Wall Modification & 2 & 6.87 \\
\hline 1614877_at & СВ002982 & TC66230 & Q9C8T5 & Proline-rich protein & Cell Wall Modification & 2 & 6.78 \\
\hline 1613330_at & CF404655 & - & Q93Z77 & Pectate lyase & Cell Wall Modification & 3 & 6.64 \\
\hline 1608120_at & CF60394I & TC70545 & Q6U619 & Pectate lyase & Cell Wall Modification & 2 & 6.6 \\
\hline 1613677_at & CB969707 & TC51953 & Q6J192 & Arabinogalactan protein & Cell Wall Modification & 2 & 6.16 \\
\hline 1619383_s_at & BQ79483I & TC66587 & Q5CCQ0 & $\beta$-galactosidase & Cell Wall Modification & 3 & 6.14 \\
\hline 1615603_at & CB346190 & TC64570 & Q8VY93 & Proline-rich protein & Cell Wall Modification & 3 & 5.75 \\
\hline 1608|80_at & CF20I469 & TC68224 & 023950 & $\begin{array}{l}\text { Endo-xyloglucan } \\
\text { transferase }\end{array}$ & Cell Wall Modification & 2 & 5.51 \\
\hline 1609593_at & CB98I468 & TC68226 & Q9LZV3 & $\begin{array}{l}\text { (I-4)- } \beta \text {-mannan } \\
\text { endohydrolase }\end{array}$ & Cell Wall Modification & 15 & 5.49 \\
\hline 1621225_at & CB974537 & TC52140 & Q9SUP5 & Polygalacturonase & Cell Wall Modification & 21 & 5.12 \\
\hline 1613415_at & AB074999 & TC45I32 & Q8W3L8 & $\begin{array}{l}\text { Xyloglucan } \\
\text { endotransglycosylase I }\end{array}$ & Cell Wall Modification & 10 & 5.1 \\
\hline 1615995_at & CF212592 & - & P24806 & $\begin{array}{l}\text { Xyloglucan } \\
\text { Endotransglucosylase } 24\end{array}$ & Cell Wall Modification & 21 & 5.02 \\
\hline 1615809_at & CB980277 & TC69342 & Q38908 & $\begin{array}{l}\text { Xyloglucan } \\
\text { endotransglucosylase } 30\end{array}$ & Cell Wall Modification & 11 & 4.87 \\
\hline 1613719_at & CF2I4562 & TC697I0 & Q7Y250 & Arabinogalactan protein & Cell Wall Modification & 2 & 4.8 \\
\hline 1613528_at & CF5I3262 & TC66769 & Q8LPS9 & Pectinesterase & Cell Wall Modification & 2 & 4.56 \\
\hline 1612668_at & CF519076 & TC61610 & Q5CHL3 & $\begin{array}{l}\text { Hydroxyproline-rich } \\
\text { glycoprotein }\end{array}$ & Cell Wall Modification & 21 & 4.32 \\
\hline 1620063_at & CB921343 & TC61082 & Q9M3U4 & $\beta-I-3$ glucanase & Cell Wall Modification & II & 4.3 \\
\hline 1611233_at & CF605724 & TC66632 & Q4W7I3 & $\beta$-xylosidase & Cell Wall Modification & 3 & 4.18 \\
\hline 1622770_at & CF209970 & TC66250 & 065186 & Cellulase & Cell Wall Modification & 13 & 4.15 \\
\hline 1609653_at & BQ797078 & TC70494 & Q9SBMI & $\begin{array}{l}\text { Hydroxyproline-rich } \\
\text { glycoprotein }\end{array}$ & Cell Wall Modification & 10 & 4.15 \\
\hline 16206|8_at & BQ794587 & TC55377 & Q8LAB2 & Proline-rich protein & Cell Wall Modification & 2 & 3.59 \\
\hline 1608799_at & BQ800204 & TC58800 & Q4ABV3 & Pectinesterase & Cell Wall Modification & 3 & 3.55 \\
\hline 1616523_s_at & CF5125I3 & TC63963 & Q8L9T8 & Arabinogalactan protein & Cell Wall Modification & 1 & 3.53 \\
\hline 1606652_at & CB969544 & TC52628 & Q8HIN7 & Polygalacturonase & Cell Wall Modification & 2 & 3.52 \\
\hline 1622353_at & BQ800489 & TC5I768 & Q5TIN5 & $\beta-6$-xylosyltransferase & Cell Wall Modification & 3 & 3.41 \\
\hline 1619659_s_at & CF405842 & TC6839I & AlllA8 & Pectate lyase & Cell Wall Modification & 14 & 3.37 \\
\hline
\end{tabular}


Table I: Transcripts (TFR pool) related cell wall metabolism categorized by the first hit in the MIPS2 catalog (Continued)

\begin{tabular}{|c|c|c|c|c|c|c|c|}
\hline 1617920_at & CF609275 & TC52380 & Q6QLN2 & Endo-I,4- $\beta$-glucanase & Cell Wall Modification & 2 & 3.31 \\
\hline 1608896_at & BQ796455 & TC59657 & Q5BM98 & $\begin{array}{l}\text { Secondary cell wall- } \\
\text { related } \\
\text { glycosyltransferase }\end{array}$ & Cell Wall Modification & 4 & 3.24 \\
\hline 1618849_at & BQ79920I & TC63732 & Q9SUP5 & Polygalacturonase & Cell Wall Modification & 21 & 3.16 \\
\hline 1610996_at & BQ794786 & TC6394I & Q43III & Pectinesterase 3 & Cell Wall Modification & 14 & 3.15 \\
\hline 1615125_at & CF372050 & TC67073 & Q5BM97 & $\begin{array}{l}\text { Secondary cell wall- } \\
\text { related } \\
\text { glycosyltransferase family } \\
\text { I } 4\end{array}$ & Cell Wall Modification & 2 & 3.09 \\
\hline 1608945_at & BQ793580 & TC54729 & P35694 & $\begin{array}{l}\text { Xyloglucan } \\
\text { endotransglycosylase }\end{array}$ & Cell Wall Modification & 15 & 3.09 \\
\hline 1607567_at & BQ795II6 & TC543I4 & Q564G6 & $\begin{array}{l}\text { Galactomannan } \\
\text { galactosyltransferase }\end{array}$ & Cell Wall Modification & 11 & 3.06 \\
\hline 1619068_at & CF2I5954 & TC60314 & Q94BII & $\begin{array}{l}\text { Xyloglucan } \\
\text { endotransglycosylase }\end{array}$ & Cell Wall Modification & 3 & 2.78 \\
\hline 1612425_at & CF37I700 & TC56348 & Q6EP64 & $\begin{array}{l}\text { Hydroxyproline-rich } \\
\text { glycoprotein }\end{array}$ & Cell Wall Modification & 11 & 2.77 \\
\hline 1616826_at & CB976610 & TC54888 & Q599J2 & $\beta-1,2$ Xylosyltransferase & Cell Wall Modification & 11 & 2.76 \\
\hline 1609138_at & CF5I9079 & TC66620 & Q1686I & $\begin{array}{l}\text { Super cysteine rich } \\
\text { protein }\end{array}$ & Cell Wall Modification & 11 & 2.74 \\
\hline 1617487_at & CD720403 & TC54500 & Q9SFF6 & Pectinacetylesterase & Cell Wall Modification & 2 & 2.69 \\
\hline 1617687_at & CB981I23 & TC57577 & Q494P2 & $\begin{array}{l}\text { Xyloglucan } \\
\text { endotransglycosylase } 2\end{array}$ & Cell Wall Modification & 21 & 2.67 \\
\hline 1606832_at & CF2I4798 & TC5I86I & Q7Y223 & $\begin{array}{l}(1-4)-\beta \text {-mannan } \\
\text { endohydrolase }\end{array}$ & Cell Wall Modification & 2 & 2.58 \\
\hline 16177/2_at & CF607664 & TC67I50 & Q39789 & $\begin{array}{l}\text { Proline-rich cell wall } \\
\text { protein }\end{array}$ & Cell Wall Modification & 2 & 2.52 \\
\hline 1617919_at & CF605842 & TC55276 & Q9SHZ2 & $\beta$-I,3-glucanase & Cell Wall Modification & 18 & 2.4 \\
\hline 1617015_at & CF209172 & TC54616 & Q7XRM8 & Pectate lyase & Cell Wall Modification & 2 & 2.34 \\
\hline 1618863_at & CF208339 & TC52953 & Q93Y12 & $\alpha$-glucosidase & Cell Wall Modification & 3 & 2.28 \\
\hline 1617939_s_at & CB910883 & TC52435 & Q41695 & Pectinacetylesterase & Cell Wall Modification & I & 2.28 \\
\hline 1616734_at & CF405846 & TC52II5 & Q6ZIF8 & $\begin{array}{l}\text { Pectin- } \\
\text { glucuronyltransferase }\end{array}$ & Cell Wall Modification & 3 & 2.28 \\
\hline 1607945_at & AF243475 & - & Q9M505 & Pectate lyase & Cell Wall Modification & 2 & 2.27 \\
\hline 161255I_at & CF605967 & TC63126 & Q9M3C5 & $\begin{array}{l}\beta-\mathrm{N}- \\
\text { acetylhexosaminidase }\end{array}$ & Cell Wall Modification & 21 & 2.26 \\
\hline 1622843_s_at & $C F 212102$ & TC65557 & Q9LVC0 & Arabinogalactan protein & Cell Wall Modification & 4 & 2.25 \\
\hline 1611230_at & AFI59I24 & - & Q9XGT3 & $\beta$-galactosidase & Cell Wall Modification & 2 & 2.25 \\
\hline 1619468_at & AY043232 & TC38735 & Q94B।6 & $\begin{array}{l}\text { Pectin methylesterase } \\
\text { PMEI }\end{array}$ & Cell Wall Modification & 12 & 2.24 \\
\hline 1610118_at & CB974025 & TC60557 & 023562 & Glucanase & Cell Wall Modification & 18 & 2.21 \\
\hline 1614868_at & CB920940 & TC64720 & Q9M0S4 & Arabinogalactan protein & Cell Wall Modification & 5 & 2.17 \\
\hline 1607528_at & AY043236 & TC6I627 & Q94B। 2 & Cellulase CELI & Cell Wall Modification & 21 & 2.11 \\
\hline 1614814_s_at & CB345895 & TC5738I & 024136 & $\mathrm{CPI} 2$ precursor & Cell Wall Modification & 13 & 2.07 \\
\hline
\end{tabular}



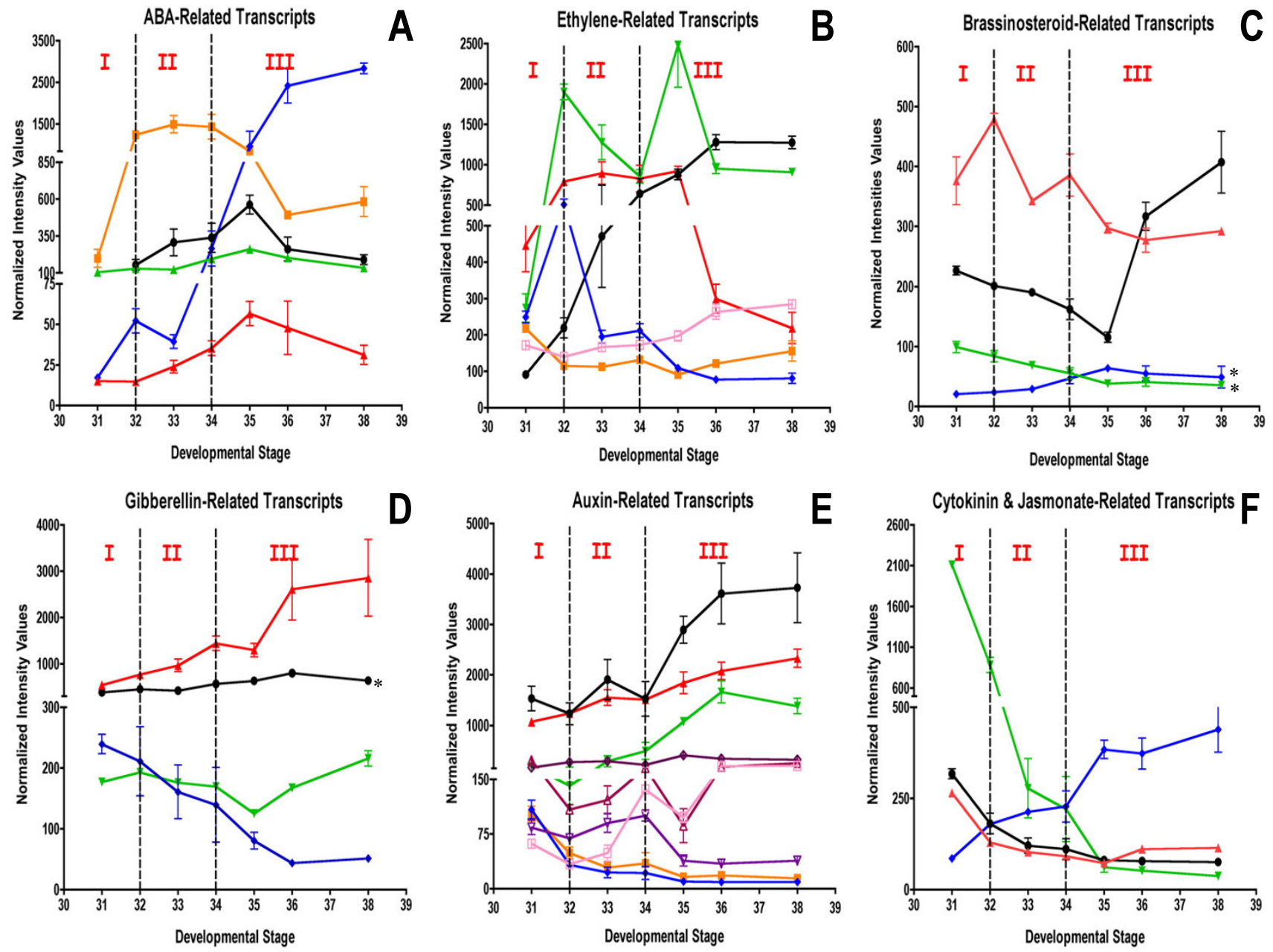

Figure 5

Expression of phytohormone transcripts. A) Black solid round (I608022_at, TC57089)-NCED isoform I, red solid triangle (I607029_at, TC5554I)-NCED isoform 4, green solid triangle (I6I4892_at, TC54474)-ABII protein phosphatase type 2C, blue solid diamond (1619802_at, TC67323)-RD22, orange solid square (I62 I 346_at, TC65 I I4)-ABI3 transcription factor. B) Black solid round (16170I2_at, TC68057)-ethylene responsive factor I, red solid triangle (I619585_at, TC62897)-ethylene induced transcription factor, green solid triangle (1621552_at, TC66829)-ethylene co-activator, blue solid diamond (I6I5952_s_at, TC56709)-aminocyclopropane carboxylic acid synthase, orange solid square (I622402_at, TC62349)-ERS I ethylene receptor, lavender open square (16185I8_at, TC55908)-EIN4/ETR5 ethylene receptor. *: transcript that does not pass the two-fold ratio. C) Black solid round (16I7572_at, TC66046)-BRHI brassinosteroid-responsive protein, red solid triangle (I6I25I6_at, TC5650 I)-BRII brassinosteroid-responsive protein, green solid triangle (1619068_at, TC603 I4)-brassinosteroid-responsive protein, blue solid diamond (I608945_at, TC54729)-BRUI brassinosteroid-responsive protein. *: transcript that does not pass the two-fold ratio. Black solid round (161818I_at, TC67464)-GIDLI receptor, red solid triangle

(162007I_at, TC56624)-GIDL2 Receptor, green solid triangle (I606777_s_at, TC56894)-GA I a gibberellin oxidase, blue solid diamond (1610610_at, TC66284)-gibberellic acid $\beta$ hydroxylase. *: transcript that does not pass the two-fold ratio. E) Black solid round (1614660_at, TC53887)-auxin responsive protein (Aux22), red solid triangle (I6I38I3_a_at, TC6554I)-auxin responsive factor 2, green solid triangle (I60959I_at, TC63193)-small auxin up RNA protein, blue solid diamond (I606566_at, TC62299)-SAUR protein, orange solid square (1616225_at, TC52772)-auxin responsive factor I8, lavender open square (1619610_at, TC56575)-IAA-amino acid hydrolase, brown open triangle (161 |479_at, CD799903)-auxin transporter, pink open triangle (1617|79_at, CF4 14958)-auxin efflux carrier, purple open diamond (1610034_at, TC59892)-auxin binding protein. F) Black solid round (160760I_at, TC6 I395)-I2-oxophytodienoate reductase, red solid triangle (I6I4324_at, CF2 I3899)-constitutive pathogen response 5 (CPR5), green solid triangle ( 620306 at, TC697I2)-cytokinin oxidase, blue solid diamond (1612955_at, TC52530)-Type-A response regulator. 
Table 2: Transcripts (TFR pool) related to phytohormone biosynthesis and response categorized by the first hit in the MIPS2 catalog

\begin{tabular}{|c|c|c|c|c|c|c|c|}
\hline Probeset ID & $\begin{array}{l}\text { GenBank } \\
\text { Annotation }\end{array}$ & VvGI5 & UniProt ID & Gene Name Description & Function & Profile & Fold Change \\
\hline 1608022_at & BQ798I05 & TC57089 & Q5SGDI & 9-cis-epoxycarotenoid dioxygenase I & ABA biosynthesis & 15 & 6.46 \\
\hline 1607029_at & CD7I6868 & TC5554I & Q8LPI4 & 9-cis-epoxycarotenoid dioxygenase 4 & ABA biosynthesis & 15 & 3.85 \\
\hline |61754I_s_at & CB342503 & TC54423 & O49814 & $\beta$-carotene hydroxylase 2 & ABA biosynthesis & 3 & 3.25 \\
\hline 161817|_s_at & BQ792407 & TC55939 & Q5SGC9 & Zeaxanthin epoxidase & ABA metabolism & 3 & 2.36 \\
\hline 1614788_at & BQ792954 & TC54II2 & Q3ZNL4 & Dehydrin la & ABA response & 11 & 26.63 \\
\hline 1609063_at & BQ799245 & TC6334I & Q4VT47 & RD-22 (ABA regulated) & $\mathrm{ABA}$ response & 3 & 11.28 \\
\hline 1621346_at & CB978597 & TC65II4 & O48620 & $A B I 3$ (ABA regulated) & $\mathrm{ABA}$ response & 14 & 7.57 \\
\hline 1614892_at & CF5II 230 & TC54474 & O82468 & Phosphatase $2 C$ (ABA regulated) & $\mathrm{ABA}$ response & 11 & 5.87 \\
\hline 1615970_at & CF405892 & TC65344 & Q7XAV5 & $\begin{array}{l}\text { Dehydration responsive element } \\
\text { binding protein }\end{array}$ & ABA response & 15 & 4.53 \\
\hline 1616735_at & CF604749 & TC51916 & 082176 & $\begin{array}{l}\text { Phosphate-induced protein (ABA } \\
\text { regulated) }\end{array}$ & $\mathrm{ABA}$ response & 16 & 3.44 \\
\hline 1607955_at & CB978I89 & TC63879 & Q9ZST5 & PII protein (ABA regulated) & $\mathrm{ABA}$ response & 12 & 3.07 \\
\hline 1621396_at & CF5I47I5 & TC51970 & Q94IB2 & Phi-2 (ABA regulated) & $\mathrm{ABA}$ response & 16 & 2.9 \\
\hline 1617417_s_at & CD798528 & TC61938 & Q9M3V0 & Phosphatase $2 \mathrm{C}$ (ABA regulated) & $\mathrm{ABA}$ response & 5 & 2.77 \\
\hline 161779|_s_at & CB0049I0 & TC70554 & Q45W74 & $\begin{array}{l}\text { Dehydration-induced protein (ABA } \\
\text { regulated) }\end{array}$ & ABA response & 4 & 2.55 \\
\hline 1609665_a_at & CB0055I5 & TC58443 & Q9M9W9 & Phosphatase-2C (ABA regulated) & $\mathrm{ABA}$ response & 20 & 2.25 \\
\hline 1609419_at & CB982969 & CB982969 & Q9S7V4 & Abscisic acid-induced protein & $\mathrm{ABA}$ response & 15 & 2.23 \\
\hline 1610937_at & BQ79288I & TC65459 & Q67WL5 & Abscisic acid-induced protein & $\mathrm{ABA}$ response & 18 & 2.2 \\
\hline 1611714_at & BQ794807 & TC53528 & Q06009 & Phosphatase $2 \mathrm{~A}$ (ABA regulated) & $\mathrm{ABA}$ response & 3 & 2.12 \\
\hline 1616882_at & CD799018 & TC53254 & Q7Y0S8 & Phi-I (ABA regulated) & $\mathrm{ABA}$ response & 3 & 2.1 \\
\hline 162104I_at & BQ794656 & TC56829 & Q9FIE3 & $\begin{array}{l}\text { Vernalization-insensitive protein } 3 \\
\text { (ABA regulated) }\end{array}$ & $\mathrm{ABA}$ response & 21 & 2.08 \\
\hline 161926I_s_at & CB982969 & TC68788 & Q5XWPI & Abscisic acid-induced protein & ABA response & 21 & 2.05 \\
\hline 1619272_at & CF373376 & TC51939 & Q94AL8 & $\begin{array}{l}\text { Cold acclimation protein (ABA } \\
\text { regulated) }\end{array}$ & $\mathrm{ABA}$ response & 3 & 2.02 \\
\hline 1619610_at & CB008850 & TC56575 & Q84XG9 & IAA-amino acid hydrolase & Auxin metabolism & 11 & 5.86 \\
\hline 1615645_at & CB969433 & TC62316 & Q8LCl6 & IAA-amino acid hydrolase & Auxin metabolism & 3 & 3.19 \\
\hline 1606566_at & CF2II64I & TC62299 & Q68IQI & Auxin-induced protein & Auxin response & 2 & 12.05 \\
\hline 160959|_at & CD79927I & TC63193 & O23089 & Auxin-regulated protein & Auxin response & 11 & 11.74 \\
\hline |6|485|_s_at & CB973279 & TC62879 & QIRYI7 & Auxin responsive Factor & Auxin response & 3 & II \\
\hline 1620662_at & CB981820 & TC59676 & Q6QUQ3 & Auxin and ethylene responsive $\mathrm{GH} 3$ & Auxin response & 11 & 10.17 \\
\hline 1614098_at & CF6084I7 & TC57853 & Q9FEL8 & AUXI like protein (influx carrier) & Auxin response & 2 & 8.8 \\
\hline 1606509_at & CB97I327 & TC5252I & Q7XEJ9 & Auxin induced protein & Auxin response & 3 & 8.29 \\
\hline 1616225_at & CB972698 & TC52772 & Q9C5W9 & Auxin response factor 2 & Auxin response & 1 & 7.3 \\
\hline 1612060_at & CB346335 & TC53973 & Q76DTI & AUXI like protein (influx carrier) & Auxin response & 7 & 6.96 \\
\hline 1616104_at & CB004955 & TC55019 & 065695 & Auxin-regulated protein & Auxin response & 15 & 6.11 \\
\hline 161200I_s_at & CF604676 & TC69850 & Q9XEY0 & Nt-gh3 (auxin and ethylene) & Auxin response & 2 & 4.69 \\
\hline 1617163_at & BQ800616 & TC5682I & Q9SHL8 & Auxin efflux carrier & Auxin response & 3 & 4.23 \\
\hline 1617513_at & CF20355I & TC52262 & Q8LAL2 & Auxin-responsive protein IAA26 & Auxin response & 2 & 3.94 \\
\hline 1613054_at & BQ794856 & TC53877 & O65695 & Auxin regulated factor & Auxin response & 3 & 3.79 \\
\hline 1621946_at & CB9754I5 & TC70724 & Q8H0E0 & PINI like auxin transport & Auxin response & 7 & 3.7 \\
\hline 1616015_at & CF607669 & TC67186 & Q2LAJ4 & Auxin response factor & Auxin response & 7 & 3.69 \\
\hline 161149|_at & CB90090I & TC5790I & Q769j4 & AtPIN3 (auxin efflux carrier) & Auxin response & 20 & 3.5 \\
\hline 1612180_at & CF608682 & TC66988 & Q6L8T9 & Auxin responsive factor 5 & Auxin response & 7 & 3.49 \\
\hline 1614660_at & CF207466 & TC53887 & PI3088 & Auxin-induced protein AUX22 & Auxin response & 11 & 3.04 \\
\hline 1617179_at & CF4I4958 & - & Q6YZX7 & Auxin efflux carrier & Auxin response & 21 & 2.91 \\
\hline 1615728_at & AY082522 & TC6098I & Q84V38 & CsIAA3 (Auxin regulated) & Auxin response & 7 & 2.66 \\
\hline 1610034_at & CB97302 & TC59892 & Q49RB8 & Auxin receptor & Auxin response & 10 & 2.45 \\
\hline 1617097_at & BQ797969 & TC67796 & Q8LER0 & Auxin efflux carrier & Auxin response & 3 & 2.41 \\
\hline 1613857_at & CD7I505I & TC55162 & $\mathrm{O} 24408$ & Auxin responsive factor & Auxin response & 2 & 2.32 \\
\hline 1607503_s_at & CF5I5267 & TC53837 & Q52QX4 & Auxin-repressed protein & Auxin response & 12 & 2.3 \\
\hline 1619658_at & CF37I85I & TC66647 & Q6QUQ3 & Auxin and ethylene responsive $\mathrm{GH} 3$ & Auxin response & 15 & 2.25 \\
\hline 1610591_at & CB923320 & TC57860 & Q3LFT5 & Auxin regulated protein & Auxin response & 10 & 2.21 \\
\hline 1620726_at & CB339504 & TC56077 & Q6YZJ0 & Auxin-regulated protein & Auxin response & 3 & 2.19 \\
\hline 1617694_at & CB972462 & TC62076 & Q93XP5 & Auxin responsive factor & Auxin response & 2 & 2.18 \\
\hline 1618394_at & CF37I644 & CF37I644 & Q94918 & Auxin growth promoter protein & Auxin response & 2 & 2.1 \\
\hline 1617572_at & CB918599 & TC66046 & Q9XF92 & $\begin{array}{l}\text { BRHI RING finger protein } \\
\text { (Brassinosteroid regulated) }\end{array}$ & $\begin{array}{l}\text { Brassinosteroid } \\
\text { response }\end{array}$ & 21 & 3.53 \\
\hline
\end{tabular}


Table 2: Transcripts (TFR pool) related to phytohormone biosynthesis and response categorized by the first hit in the MIPS2 catalog

\begin{tabular}{|c|c|c|c|c|c|c|c|}
\hline 1620306_at & CF404552 & TC697I2 & Q5ZAY9 & Cytokinin oxidase & Cytokinin response & 3 & 57.36 \\
\hline 161007I_at & BQ797708 & TC58750 & Q39802 & Cytokinin induced message & Cytokinin response & II & 13.16 \\
\hline 1619945_at & CB345883 & TC61250 & Q84N27 & Cytokinin repressed protein & Cytokinin response & 19 & 3.05 \\
\hline 1622308_at & CF210289 & TC63310 & Q8S933 & $\begin{array}{l}\text { I-aminocyclopropane-I-carboxylate } \\
\text { synthase }\end{array}$ & $\begin{array}{l}\text { Ethylene } \\
\text { biosynthesis }\end{array}$ & 2 & 11.84 \\
\hline 1615952_s_at & CF2I564I & TC56709 & Q84X67 & $\begin{array}{l}\text { I-aminocyclopropane-I-carboxylic } \\
\text { acid oxidase }\end{array}$ & $\begin{array}{l}\text { Ethylene } \\
\text { biosynthesis }\end{array}$ & 3 & 6.56 \\
\hline 1609683_at & CF604955 & TC65735 & Q5U8L6 & Ethylene responsive factor 2 & Ethylene response & 12 & 22.64 \\
\hline 1617012_at & CD802399 & TC68057 & $\mathrm{PI} 6146$ & Ethylene-responsive element & Ethylene response & 11 & 14.05 \\
\hline 1621552_at & BM4375I0 & TC66829 & Q9LV58 & $\begin{array}{l}\text { Ethylene-responsive transcriptional } \\
\text { co activator }\end{array}$ & Ethylene response & 21 & 9.08 \\
\hline 1619585_at & CD800299 & TC62897 & Q75UJ4 & Ethylene responsive factor & Ethylene response & 13 & 4.21 \\
\hline 1611910_s_at & AY395745 & TC63214 & Q6TKQ3 & Ethylene responsive factor 4 & Ethylene response & 3 & 4.13 \\
\hline 1609990_at & CB009298 & TC63214 & Q6TKQ3 & Ethylene responsive factor 2 & Ethylene response & 3 & 4.12 \\
\hline |6085II_at & CB342877 & TC62587 & Q6RZW7 & Ethylene responsive factor 5 & Ethylene response & 15 & 3.72 \\
\hline 1609780_at & CA810742 & TC55438 & Q94E74 & Ethylene responsive factor 6 & Ethylene response & 3 & 3.22 \\
\hline 1612699_at & BQ7986I4 & BQ7986I4 & Q9XIA5 & $\begin{array}{l}\text { Ethylene-forming-enzyme-like } \\
\text { dioxygenase }\end{array}$ & Ethylene response & 12 & 2.75 \\
\hline 1619178_at & CB349106 & TC54200 & Q94E74 & Ethylene responsive 6 & Ethylene response & 19 & 2.62 \\
\hline 1613799_at & CF5I72II & TC55673 & Q6RZW8 & Ethylene responsive factor 4 & Ethylene response & 21 & 2.5 \\
\hline 1622402_at & CD799344 & TC62349 & Q84PH6 & Ethylene receptor (EIN4) & Ethylene response & 20 & 2.4 \\
\hline 1609559_at & CF2I5263 & TC58568 & Q94AW5 & Ethylene-responsive element & Ethylene response & 21 & 2.34 \\
\hline 1606623_at & BQ797592 & TC70037 & Q9LVS8 & EREBP-4 & Ethylene response & 3 & 2.28 \\
\hline 161292I_at & CF5I4773 & TC57403 & Q9LVS8 & EREBP-4 & Ethylene response & 3 & 2.26 \\
\hline 1618213_at & CF203873 & CF203873 & Q9SWV2 & ER6 (Ethylene regulated) & Ethylene response & 3 & 2.16 \\
\hline 1611657_at & CF20886I & TC67832 & O64588 & $\begin{array}{l}\text { GH3 Root formation (gibberellin } \\
\text { regulated) }\end{array}$ & GA response & 3 & 8.07 \\
\hline 1610607_at & CF37I650 & TC66III & Q49RB3 & GASA & GA response & 3 & 6.98 \\
\hline 1621228_at & BQ798029 & TC52322 & Q6S5L6 & GAI protein (Gibberellin regulated) & GA response & 7 & 2.52 \\
\hline 1610610_at & CA810332 & TC66284 & Q9ZQA5 & Putative gibberellin $\beta$-hydroxylase & $\begin{array}{l}\text { Gibberellin } \\
\text { metabolism }\end{array}$ & 10 & 3.52 \\
\hline I62007I_at & BQ8002/4 & TC56624 & Q9LYCI & Gibberellin receptor & $\begin{array}{l}\text { Gibberellin } \\
\text { response }\end{array}$ & 11 & 5.3 \\
\hline 161818I_at & CF5I 2673 & TC67464 & Q9MAA7 & Gibberellin receptor 2 & $\begin{array}{l}\text { Gibberellin } \\
\text { response }\end{array}$ & 16 & 2.1 \\
\hline 1622456_at & CF609276 & TC66424 & Q7PCB5 & Phytosulfokine & Growth factor & 3 & 9.3 \\
\hline 1616312_at & CD720049 & TC61028 & Q7PCA0 & Phytosulfokine peptide precursor & Growth factor & 12 & 3.69 \\
\hline 1607170_s_at & CB917184 & TC667I7 & Q7PCAI & Phytosulfokine & Growth factor & 21 & 2.07 \\
\hline 161202I_at & CF213898 & CF213898 & Q7EYF8 & Phytosulfokine receptor & $\begin{array}{l}\text { Growth factor } \\
\text { response }\end{array}$ & 3 & 4.06 \\
\hline I60760I_at & CF209956 & TC61395 & Q76DL0 & 12-oxophytodienoate reductase & JA metabolism & 2 & 4.2 \\
\hline 1619407_s_at & CA809049 & TC67I04 & Q76DL0 & 12-oxophytodienoate reductase & JA metabolism & 21 & 2.92 \\
\hline 1620308_at & CF208037 & TC57918 & Q38944 & Steroid 5-alpha-reductase & $\begin{array}{l}\text { Lipid, fatty acid and } \\
\text { isoprenoid } \\
\text { metabolism }\end{array}$ & 3 & 3.5 \\
\hline I6I394I_at & CA8I853I & TC6I6II & Q7X9G5 & Lipoxygenase & $\begin{array}{l}\text { Lipid, fatty acid and } \\
\text { isoprenoid } \\
\text { metabolism }\end{array}$ & 1 & 2.99 \\
\hline 1618940_at & CF2I 2858 & TC64939 & Q8W250 & $\begin{array}{l}\text { I-deoxy-D-xylulose } 5 \text {-phosphate } \\
\text { reductoisomerase }\end{array}$ & $\begin{array}{l}\text { Lipid, fatty acid and } \\
\text { isoprenoid } \\
\text { metabolism }\end{array}$ & 3 & 2.03 \\
\hline 1613678_at & CB97I023 & TC54495 & Q9M2G7 & Phosphatase & $\begin{array}{l}\text { Phosphate } \\
\text { metabolism }\end{array}$ & 3 & 2.07 \\
\hline 1612552_at & CA818350 & CA8I8350 & Q9C9W8 & $\begin{array}{l}\text { S-adenosyl-L-methionine:salicylic acid } \\
\text { carboxyl methyltransferase }\end{array}$ & SA response & 11 & 6.99 \\
\hline 1618457_at & CF205I 25 & CF205I 25 & Q9M6E7 & $\begin{array}{l}\text { UDP-glucose:salicylic acid } \\
\text { glucosyltransferase }\end{array}$ & SA response & 12 & 2.19 \\
\hline 1619377_at & CF372632 & TC68498 & Q5Z825 & avrRpt2-induced AIG2 protein & SA response & 12 & 2.06 \\
\hline
\end{tabular}

stage 35 around the start of véraison (Figure 5A). Both NCED1 (1608022_at, TC57089) and NCED4 (1607029_at, TC55541) had similar expression patterns, but differed significantly in their relative trancript abundance. A transcript (1614892_at, TC54474) encoding ABI1 (protein phosphatase 2C) showed an expression 
pattern like that of the NCED genes, but was more highly correlated with NCED4 than NCED1. The RD22 gene (1619802_at, TC67323), a dehydration-responsive protein, displayed a very large increase in abundance at véraison that continued to increase during berry maturation, whereas another transcript (1621346_at, TC65114) encoding an ABI3/VP1 (ABscisic acid Insensitive 3/ViviParous 1) transcription factor showed highest transcript abundance during the lag phase.

\section{Ethylene}

Traditionally, wine grape has been considered a non-climacteric fruit, however, there are studies that indicate that ethylene plays an important role in berry development and ripening [13] and is required for increased berry diameter and ripening processes, such as anthocyanin biosynthetic gene expression and accumulation [46,47]. In addition, ethylene appears to be involved in controlling the expression of an alcohol dehydrogenase gene from grape [48]. Furthermore, some inhibitors of ethylene biosynthesis can delay berry ripening [49]. Ethylenerelated transcripts displayed some very unique and intriguing patterns of expression (Figure 5B) indicating that this signaling pathway is differentially expressed along berry development. One transcript (1617012_at, TC68057) encoding a putative Ethylene Response Factor 1 (ERF1), a putative ethylene output gene, displayed a steady increase in abundance with maximal expression at ripening (Figure 5B) indicating a potential post-véraison role for this signaling pathway. An ethylene-induced transcription factor (1619585_at, TC62897) exhibited transcript accumulation during the lag (E-L stages 32 to 34 ) and early véraison (E-L stage 35) stages of development. A putative ethylene co-activator (1621552_at, TC66829) protein displayed biphasic peak transcript abundance at E-L stages 32 and 35 . The transcript abundance of ACC oxidase (1615952_s_at, TC56709), the enzyme responsible for the last step in ethylene biosynthesis, was highest at E-L stage 32, the start of the lag phase, and then declined throughout the remainder of berry development. Interestingly, the transcript abundance of an ethylene receptor ERS1 (1622402_at, TC62349) and EIN4/ETR5 (1618518_at, TC55908) were at their lowest during E-L stages 32 to 33 until véraison, but then increased at a later stage (E-L stage 38 ). Ethylene pathway activation in grape berry appears to occur within a three week period of berry development (weeks 6 to 8 after anthesis; E-L stages 30 to 33 ) when the highest ethylene (ACC) content and transcript abundance of ACC oxidase were detected in Cabernet Sauvignon [46]. This hypothesis is supported by the observation that application of exogenous ethylene 8 weeks after anthesis hastened the ripening of the grape berries and resulted in a decrease in average cell size. In contrast, if the same ethylene treatment was applied dur- ing earlier stages of berry development (at 4, 5, 6 or 7 weeks), maturation was delayed [47].

According to the Arabidopsis model of ethylene signaling, reduced expression of transcripts and activity of receptors increases the sensitivity to ethylene, whereas increased receptor expression and activity decreases sensitivity [50]. In tomato, the expression of most genes encoding ethylene receptors increases during fruit development. In parallel, high levels of ethylene are expressed to counterbalance the negative effect of increased receptor expression on the ethylene signaling pathway [51]. In grape berry, the slight decreases observed in ethylene receptor transcript expression occurring between E-L stages 31 and 32 and the peak of ethylene accumulation during this same period, indicate a higher sensitivity to ethylene during the early stages of berry development. This would be expected to lead to a greater activation of the ethylene signaling pathway prior to véraison.

As in grape berry, strawberry is able to produce significant levels of ethylene during fruit development, but not to the same extent as climacteric fruits. Recently, three ethylene receptors have been identified in strawberry [52]. Two of them (FaEtr1 and FaErs1) display the same pattern of expression during fruit development as those observed for ERS1 ethylene receptor during grape berry development. In addition, the highest rates of ethylene production in strawberry were detected in very young green fruits. Following this, the hormone decreases continuously until the White stage of fruits. Following this stage, ethylene showed a slight but steady increase for the remainder of development. When considered together, the similarities of expression of ethylene receptors during fruit development for both grapes and strawberries coupled with the concomitant ethylene production during the early steps of fruit development indicate a conserved mechanism for ethylene perception between these fruits prior to ripening.

\section{Brassinosteroids}

Brassinosteroids (BR) have recently been implicated in playing an important role in berry development [53]. Castasterone concentrations are low during the early stages of berry development and then increase at véraison [53]. Brassinosteroids have been shown to increase cell size [54] indicating that berry enlargement may be affected by castasterone levels. BRH1 RING finger protein (1617572_at, TC66046) transcript abundance, which is known to be down-regulated by exogenous application of $\mathrm{BR}$, decreased during E-L stages 31 to 35, but increased in fully mature berries (Figure $5 \mathrm{C}$ ). The transcript abundance of the Brassinosteroid Receptor 1 gene (BRI1, 1612516_at, TC56501) peaks at the start of the lag phase (E-L stage 32) and then declines thereafter. The transcript abundance of BRU1 (1608945_at, TC54729), which is a 
BR-responsive transcript encoding a xyloglucan endotransglycosylase (XET), showed a transient increase in abundance at véraison. In the same family, transcripts for another BR-responsive protein (1619068_at, TC60314) declined with berry development. Clearly, there are many significant changes in transcript abundance that are associated with brassinosteroid responses during berry development.

\section{Gibberellins}

Very little is known about the role of gibberellin (GA) in grape berry development except a possible role in cell enlargement. Biologically active concentrations of GA are high in flowers and in fruits just after anthesis, but then drop to lower levels over the course of berry development $[53,55]$. There is a second peak of active GA at the start of the lag phase and it is 77 times higher in the seed compared to the berry mesocarp [56]. The transcript abundance of two putative GA receptors, GIDL1 and GIDL2 (1618181_at, TC67464; 1620071_at, TC56624, respectively), increased during berry development (Figure 5D). Interestingly, the transcript abundance of the GA signaling pathway repressor, GAI1 (1606777_s_at, TC56894), declines transiently at véraison. The transcript abundance of a putative GA $\beta$-hydroxylase (1610610_at, TC66284) declines over the course of berry development (Figure 5D) more or less coincident with the known accumulation pattern of $\mathrm{GA}_{1}$ in developing berries.

\section{Auxins}

The mechanisms by which the phytohormone indole-3acetic acid (IAA) regulates berry development are complex and not fully understood. Increased auxin production produced through the action of an ovule-specific auxinsynthesizing transgene enhanced fecundity in grapes [57]. Earlier reports indicated that auxin concentrations were high during early Phase I and declined following véraison [55] consistent with the role of this phytohormone in promoting cell division and expansion during Phase I. Treatment of grape berries with synthetic auxin-like compound, benzothiazole-2-oxyacetic acid (BTOA) delayed ripening [45]. A more recent study showed that auxin concentrations remain relatively constant over the course of berry development [53].

Our data indicate that there are numerous transcript responses to auxin (Figure 5E). The transcript abundance of Aux22 (1614660_at, TC53887), which forms heterodimers with auxin response factors (ARF) in order to repress auxin responses, increased after véraison (Figure 5E). Transcripts for both Auxin Response Factor 2 (ARF2, 1613813_a_at, TC65541) and a Small Auxin Up RNA protein (SAUR) (1609591_at, TC63193) increased after véraison, whereas transcripts for a different SAUR transcript (1606566_at, TC62299) and an Auxin-induced
Response Factor, ARF18 (1616225_at, TC52772) both declined in a very similar pattern during berry development. A transcript (1619610_at, TC56575) encoding IAAamino acid hydrolase, which is involved in IAA homeostasis, was highly expressed during the later stages of berry development (Figure 5E). The synthesis and hydrolysis of IAA conjugates, which function in both permanent inactivation and temporary storage of auxin [58], may play an important role in the control of IAA concentrations as berry development progresses. IAA-amino acid hydrolase may provide for local concentrations of auxins within the berries to promote mesocarp cell enlargement. Several transcripts (1611479_at, CD799903; 1617179_at, CF414958; 1610034_at, TC59892) related to auxin transport and perception also displayed increased abundance at the onset of verraison. Given the importance of auxinmediated processes in developing berries, more research needs to be conducted to elucidate the mode of action of auxin signaling and response pathways.

\section{Methyl jasmonate and cytokinins}

Methyl jasmonate (MeJA) is known to promote the synthesis and accumulation of terpenes and resveratrol in berry cell cultures $[59,60]$, however, its effects in vivo are not well understood. The transcript abundance of 12-oxophytodienoate reductase (12-OPR) (1607601_at, TC61395), which is involved in jasmonate biosynthesis [61], and a constitutive pathogen-response 5 protein (1614324_at, CF213899), both decreased with berry development (Figure 5F). Less is known about the role of cytokinins in berry development. The transcript abundance of cytokinin oxidase (1620306_at, TC69712), which degrades cytokinin [62], decreased over berry development, whereas a known cytokinin-response regulator, a Type-A response regulator (1612955_at, TC52530), showed a steady increase in transcript abundance over berry development (Figure 5F).

\section{New candidates genes associated with calcium signaling, flavonoid transport and flavor}

Calcium has many essential roles in plant growth and development [63], however, the role of calcium signaling in grape berry development is largely unexplored. Recently, an ABA-responsive calcium-dependent protein kinase (CDPK) was described that was specifically expressed in the seed and flesh of berries with increased transcript abundance over berry development and ripening [64]. In the current study, a large number of genes with functions related to calcium sequestration, transport and signaling were found to display developmentally regulated expression patterns (Figure 6A; Table 3).

Calcium homeostasis within the cytosol is tightly controlled by membrane spanning $\mathrm{Ca}^{2+}$-ATPases and $\mathrm{H}^{+} / \mathrm{Ca}^{2+}$ exchangers, which typically maintain low concentrations 


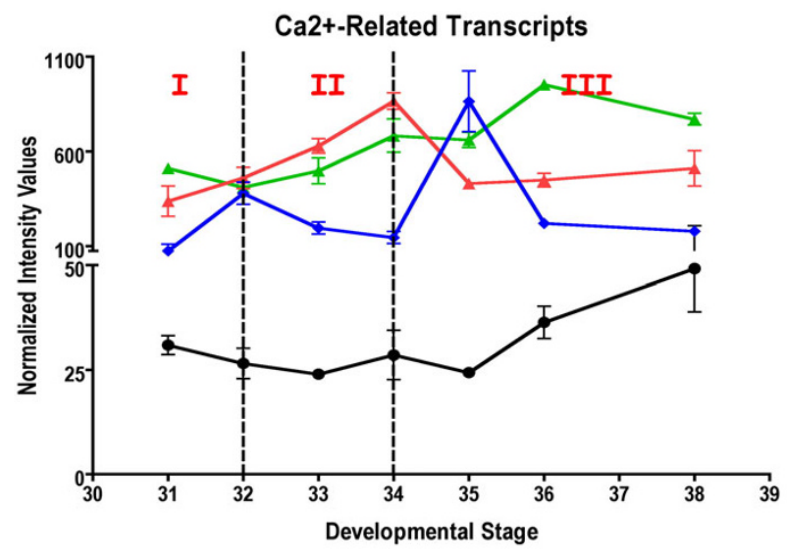

A

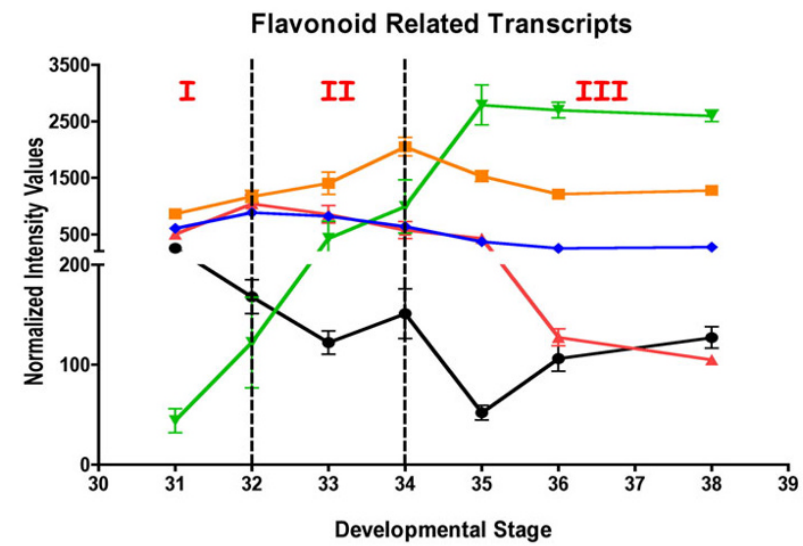

B

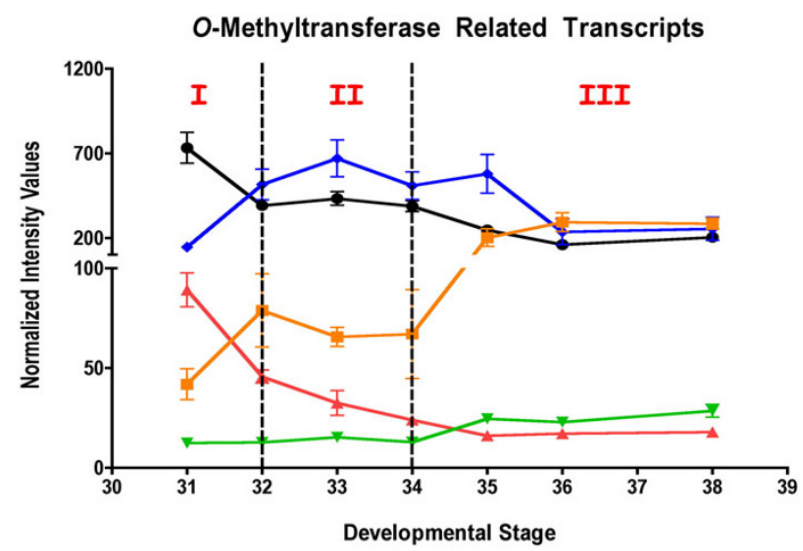

\section{Figure 6}

Expression of potential candidates Unigenes. A) Black solid round ( $16 \mid 4028$ at, TC67285)-cation-transporting ATPase, red solid triangle (1622073_at, CF4042 I4)-calcium-transporting ATPase, green solid triangle (1617237_s_at, TC66680)-Ca ${ }^{2+}$ I $\mathrm{H}^{+}$exchanger, blue solid diamond (1618587_at, TC64370)-calmodulin-repressor of gene silencing. B) Black solid round (1619917_s_at, TC69505)-glutathione-S-transferase, red solid triangle (I609870_at, TC58286)-glutathione-S-transferase conjugating ATPase, green solid triangle (I607560_at, TC62 I62)-multi-drug secondary transporter like protein (MATE), blue solid diamond (16II09I_s_at, TC54724)-VvMYBPAI, orange solid square (16I8504_at, TC6I7I3)-MYC transcription factor. C) Black solid round (I608603_at, TC56956)-phloroglucinol O-methyltransferase, red solid triangle (I6I3542_at, TC62584) Omethyltransferase, green solid triangle (1620469_at, CF209780)-O-methyltransferase, blue solid diamond (I6I6348_at, TC52353)-S-adenosyl-L-methionine:benzoic acid/salicylic acid carboxyl methyltransferase orange solid square (I6I2552_at, TC57I70)-S-adenosyl-L-methionine:salicylic acid carboxyl methyltransferase. 
Table 3: Transcripts (TFR pool) related to calcium categorized by the first hit in the MIPS2 catalog

\begin{tabular}{|c|c|c|c|c|c|c|c|}
\hline Probeset ID & $\begin{array}{l}\text { GenBank } \\
\text { Annotation }\end{array}$ & VvGI5 & $\begin{array}{l}\text { UniProt } \\
\text { ID }\end{array}$ & Gene Name Description & Function & Profile & Fold Change \\
\hline 1616662_at & CF404703 & TC59643 & Q9LIK7 & $\mathrm{Ca} 2+/ \mathrm{ATPase}$ & Ca transport & 3 & 27.96 \\
\hline 1617237_s_at & CF207946 & TC66680 & O64455 & $\mathrm{Ca} 2+/ \mathrm{H}+$ exchanger (VCAXI) & Ca transport & 14 & 2.56 \\
\hline 1614028_at & CB976052 & TC62785 & Q7X8B5 & Ca2+-transporting ATPase 8 & Ca transport & 16 & 2.32 \\
\hline 1619731_at & CB972437 & CB972437 & Q93YX7 & Type IIB calcium ATPase & Ca transport & 21 & 2.2 \\
\hline 1622073_at & CF4042I4 & CF4042I 4 & Q9LIK7 & Calcium-transporting ATPase 13 & Ca transport & 5 & 2.05 \\
\hline 1615486_at & CF4I5476 & TC6935I & Q5D6H2 & Cyclic Nucleotide-Gate Channel 2 & Ion channel & 3 & 8.21 \\
\hline |62159|_at & CB981532 & TC66482 & Q94AS9 & Cyclic nucleotide-gated ion channel 4 & lon channel & 10 & 3.01 \\
\hline 1609527_at & CD802146 & TC64II7 & Q6ZHE3 & Cyclic nucleotide-binding transporter I & Ion channel & 8 & 2.06 \\
\hline 1613268_at & CB342482 & TC532I3 & 065717 & Cyclic nucleotide-gated ion channel I & lon channel & 12 & 2.05 \\
\hline 1614456_at & BQ797488 & BQ797488 & Q8L706 & $\mathrm{Ca} 2+-d e p e n d e n t$ lipid-binding protein & Lipid binding & 11 & 3.83 \\
\hline 1614582_at & BQ799084 & BQ799084 & Q8LJ85 & Calreticulin & $\begin{array}{l}\text { Protein folding and } \\
\text { stabilization }\end{array}$ & 12 & 2.37 \\
\hline 1611917_at & CB972I64 & TC58290 & Q39817 & Calnexin & $\begin{array}{l}\text { Protein folding and } \\
\text { stabilization }\end{array}$ & I & 2.31 \\
\hline |6|229|_at & CB347450 & TC67746 & P93508 & Calcium-binding protein & $\begin{array}{l}\text { Protein folding and } \\
\text { stabilization }\end{array}$ & 3 & 2.2 \\
\hline 1622324_at & CF568845 & TC63952 & Q39817 & Calnexin & $\begin{array}{l}\text { Protein folding and } \\
\text { stabilization }\end{array}$ & 1 & 2.04 \\
\hline 1612443_at & CF2III5I & TC68392 & Q7X996 & CBL-interacting protein kinase 20 & Signal transduction & 11 & 12.97 \\
\hline 1610295_at & BQ797947 & TC57947 & Q8WID5 & CBL-interacting protein kinase 5 & Signal transduction & 4 & 12.35 \\
\hline 1618587_at & CF5I8I3I & TC64370 & Q9AXG2 & Calmodulin & Signal transduction & 21 & 11.43 \\
\hline 1618447_at & CA8I5I4I & TC53225 & Q6ETM9 & CBL-interacting protein kinase $2 \mathrm{I}$ & Signal transduction & 3 & 7.75 \\
\hline 1611127 at & CF510878 & TC64442 & Q8L3R2 & Calmodulin & Signal transduction & 12 & 5.33 \\
\hline 1610922_at & CF4043I5 & TC68II6 & QISFZ7 & CBL-interacting protein kinase $2 \mathrm{I}$ & Signal transduction & 3 & 3.43 \\
\hline 1606980_at & CF2II606 & TC6950I & Q008R9 & Calcium sensor homolog & Signal transduction & 2 & 3.23 \\
\hline 1618045_at & CF216119 & TC53057 & Q676UI & CBL-interacting protein kinase 20 & Signal transduction & 21 & 2.9 \\
\hline 1611172 at & CB003645 & TC52484 & Q8LK24 & SOS2-like protein kinase & Signal transduction & 16 & 2.81 \\
\hline 1612269_at & CB345885 & TC53895 & Q3HRN8 & Calcineurin B & Signal transduction & 13 & 2.74 \\
\hline 1606859_at & CF5I888I & CF5I888I & Q3HRN8 & Calcineurin B & Signal transduction & 13 & 2.74 \\
\hline 1613576_s_at & CF201676 & TC60874 & P62200 & Calmodulin $1 / 1 \mathrm{I} / 16$ & Signal transduction & 11 & 2.7 \\
\hline 1622351_at & CA810859 & TC60874 & P62200 & Calmodulin $1 / 1 \mathrm{l} / 16$ & Signal transduction & 11 & 2.26 \\
\hline 1611555_at & CB971903 & TC54I54 & Q9SS31 & Calmodulin-related protein 2 & Signal transduction & 13 & 2.22 \\
\hline 1608587_at & CD799705 & TC62I5I & Q5D875 & $\begin{array}{l}\text { Calcium-dependent protein kinase } \\
\text { CDPK I } 444\end{array}$ & Signal transduction & 10 & 2 \\
\hline 1614600_s_at & CF2I3754 & TC52I50 & Q9ZT86 & Calcium-binding protein & Unclassified protein & 7 & 2.89 \\
\hline 1616580_at & CF206767 & TC5559I & Q84Y 18 & CAX-interacting protein 4 & Unclassified protein & 11 & 2.63 \\
\hline
\end{tabular}

of $\mathrm{Ca}^{2+}$ in the cytosol and restore this concentration following signaling-related transient changes in calcium levels. Transcripts encoding plasma membrane $\mathrm{Ca}^{2+}$-ATPase genes (1614028_at, TC62785; 1622073_at, CF404214), which are closely related to ACA8 and ACA13, respectively, in Arabidopsis thaliana, showed increased transcript abundance during E-L stages 33 and 34 and in later developmental stages. Interestingly, ABA markedly and rapidly stimulates the expression of the ACA8 gene in cell cultures of Arabidopsis thaliana [65]. A tonoplast $\mathrm{Ca}^{2}+/ \mathrm{H}^{+}$exchanger (1617237_s_at, TC66680), which is a close homolog of CAX3 from A. thaliana and plays a key role in cytosolic $\mathrm{Ca}^{2+}$ homeostasis [66], showed a transient increase in transcript abundance at E-L stages 34, indicating a possible role for calcium signaling around véraison.

ABA accumulates until two weeks after the beginning of véraison before decreasing later in berry development
[67]. Thus, it is likely that ABA is directly or indirectly involved in the control of $\mathrm{Ca}^{2+}$ signaling and homeostasis events, particularly around véraison.

The increased expression of several Unigenes encoding calmodulin or calcium interacting protein kinases (see Table 3) supports this hypothesis [68]. One Unigene encoding a calmodulin-related suppressor of gene silencing (1618587_at, TC64370) displayed a pronounced pattern with two peaks of expression at E-L stage 32 and at EL stage 35 corresponding to two transitions of berry development (Phases I to II and Phases II to III). This Unigene displayed a 10 -fold change in its transcript abundance across berry development and may be involved in the suppression of posttranscriptional gene silencing (PTGS) by interacting with a proteinase known to suppress PTGS in plants [69]. This correlation indicates a possible role for calcium in regulating the activity of the PTGS mecha- 
nisms. To date, only one paper reported the possibility of the involvement of PTGS in the regulation of gene expression during plant development [70]. Further investigations are necessary to evaluate the real impact of this Unigene in the triggering of veraison.

Phenolic compounds, derived from flavonoids (anthocyanins, tannins and flavonols), are the major wine constituents responsible for organoleptic properties such as color and astringency. Twenty-one Unigenes encoding biosynthetic enzymes of the general phenylpropanoid and flavonoid pathways were found to exhibit differential mRNA expression patterns across berry development (Table 4). The vast majority of these genes are expressed predominantly in the skin [71].

The mechanisms by which anthocyanins accumulate in the vacuole of grape berry skin cells during Phase III are not fully understood. These compounds must be transported from the site of synthesis in the cytosol to their final destination, the vacuole. Several models have been proposed for sequestering anthocyanins in the vacuole in Arabidopsis thaliana. One model [72] indicates the action of a glutathione-S-transferase (GST) in facilitating the transfer of anthocyanins into the vacuole. Another model indicates that a transporter of the multidrug-resistance- associated protein family could facilitate the transport of an anthocyanin-GST complex into the vacuole [73]. Here, the Unigene transcript encoding a GST (1619917_s_at, TC69505; Figure 6B) displayed a 63-fold increase in abundance during the stages in berry development in which flavonoids accumulate (Figure 6B). This Unigene is closely related to a GST homolog known to be involved in anthocyanin sequestration [74]. This Unigene also displays a skin-specific expression pattern [71], which is consistent with the tissue localization of anthocyanins. A Unigene homologous to a glutathione-S-conjugate transporting ATPase (1609870_at, TC58286) showed a peak of expression at véraison (E-L stage 34 ). While not yet characterized in detail, this Unigene belongs to the ABC transporter sub-family, members of which are known to transport anthocyanins [74]. The putative multi-drug transporter (1607560_at, TC62162), which is known to be involved in the sequestration of tannins into vacuoles [75], exhibited peak transcript abundance at E-L stage 32 followed by a decline, and is consistent with the pattern of maximal tannin accumulation that occurs a few weeks before véraison.

Specific members of the MYB transcription factor family play critical roles in the regulation of flavonoid metabolism during grape berry development [76]. We detected

Table 4: Transcripts (TFR pool) related to flavonoid metabolism categorized by the first hit in the MIPS2 catalog within specific subsections of the flavonoid pathway

\begin{tabular}{|c|c|c|c|c|c|c|c|}
\hline Probeset ID & $\begin{array}{l}\text { GenBank } \\
\text { Annotation }\end{array}$ & VvGI5 & UniProt ID & Gene Name Description & Function & Profile & Fold Change \\
\hline 161717|_s_at & AF00037I & TC51696 & O22303 & $\begin{array}{l}\text { UDP glucose:flavonoid 3-o- } \\
\text { glucosyltransferase (UFGT) }\end{array}$ & Anthocyanin Pathway & 11 & 46.79 \\
\hline |6|444|_at & BQ79824I & TC57653 & Q9SWY6 & Anthocyanidin synthase (ANS) & Anthocyanin Pathway & 11 & 12 \\
\hline 1618112 at & CB97I 725 & TC70789 & Q9LTA3 & $\begin{array}{l}\text { Anthocyanidin-3-glucoside } \\
\text { rhamnosyltransferase }\end{array}$ & Anthocyanin Pathway & 3 & 9.39 \\
\hline 1611309_at & CF210457 & TC58629 & Q8HIRI & Dihydroflavonol 4-reductase (DFR) & Common Pathway & 19 & 7.36 \\
\hline 1611739_at & CF403783 & TC64266 & $\mathrm{Q} 8 \mathrm{H} 224$ & Flavonoid 3'-hydroxylase $\left(\mathrm{F}^{\prime}{ }^{\prime} \mathrm{H}\right)$ & Common Pathway & 2 & 5.68 \\
\hline 1620675_at & CB969894 & TC51699 & P93799 & Dihydroflavonol 4-reductase (DFR) & Common Pathway & 3 & 5.21 \\
\hline 1617019_at & BQ800456 & TC67I73 & O80407 & Chalcone synthase (CS) & Common Pathway & 3 & 5.17 \\
\hline 1607739_at & CF4I5693 & TC70298 & P41090 & Flavanone 3-hydroxylase $(\mathrm{F} 3 \mathrm{H})$ & Common Pathway & 3 & 2.93 \\
\hline 1608379_at & CF202029 & TC40489 & Q8H8H7 & Flavanone 3-hydroxylase $(\mathrm{F} 3 \mathrm{H})$ & Common Pathway & 21 & 2.55 \\
\hline 1607732_at & AF020709 & TC63806 & 022519 & Chalcone synthase (CS) & Common Pathway & 3 & 2.48 \\
\hline 160876I_at & CB982029 & TC5333I & Q9FLV0 & Flavanone 3-hydroxylase $(\mathrm{F} 3 \mathrm{H})$ & Common Pathway & 18 & 2.02 \\
\hline 1611542_at & CB971080 & TC5I69I & P433II & Polyphenol oxidase (PPO) & Flavonoid Catabolism & 3 & 28.9 \\
\hline |62265|_at & CF2I5945 & TC58764 & P93622 & Polyphenol oxidase (PPO) & Flavonoid Catabolism & 5 & 3.79 \\
\hline |60879|_at & CB978059 & TC66577 & Q84TMI & Flavonol synthase (FLS5) & Flavonol Pathway & 3 & 5.12 \\
\hline |62105I_at & CN006197 & - & Q40285 & Flavonol 3-O-glucosyltransferase & Flavonol Pathway & 13 & 3.94 \\
\hline 161540I_at & CB342555 & TC5533I & Q40285 & Flavonol 3-O-glucosyltransferase & Flavonol Pathway & 15 & 2.43 \\
\hline 1618155_at & CD004374 & TC54048 & Q40288 & Flavonol 3-O-glucosyltransferase 6 & Flavonol Pathway & 10 & 2.27 \\
\hline 1612134_at & CF204393 & TC53206 & Q5FB34 & Anthocyanin reductase (ANR) & $\begin{array}{l}\text { Proanthocyanidin } \\
\text { Pathway }\end{array}$ & 3 & 34.12 \\
\hline 1615174_s_at & CD0III073 & TC6874I & Q4W2K6 & $\begin{array}{l}\text { Leucoanthocyanidin reductase } 2 \\
\text { (LAR2) }\end{array}$ & $\begin{array}{l}\text { Proanthocyanidin } \\
\text { Pathway }\end{array}$ & 13 & 4.08 \\
\hline 16082/2_at & CKI38I22 & TC54322 & Q84V83 & $\begin{array}{l}\text { Leucoanthocyanidin reductase } 2 \\
\text { (LAR2) }\end{array}$ & $\begin{array}{l}\text { Proanthocyanidin } \\
\text { Pathway }\end{array}$ & 13 & 3.52 \\
\hline
\end{tabular}


four transcripts encoding MYB transcription factors that have been previously characterized in grape berry (see Table 4) [77-80]. VvMYBPA1 (1611091_s_at, TC54724) regulates the proanthocyanidin (condensed tannins) pathway in the grape berry [77]. In the Shiraz cultivar, VvMYBPA1 peak expression appears to occur during E-L stages 34 and 35 in the skin and seeds, whereas, in Cabernet Sauvignon this gene is expressed at an earlier developmental stage (E-L stages 32) (Figure 6B). Such differences are likely to be cultivar-dependent. In the same way, the MYC family of transcription factors also plays a key role in regulation of the anthocyanin pathway. One MYC transcription factor transcript (1618504_at, TC61713), which shares strong amino acid sequence identity with MYC genes known to be involved in the regulation of anthocyanin production [81], displayed a pattern of transcript accumulation that decreased from the beginning of berry development until E-L stage 35 and then increased for the remainder of fruit development (Figure 6B). Furthermore, this Unigene is preferentially expressed in the skin [71]. These expression patterns correlate well with the accumulation of anthocyanins and proanthocyanins.

In grape berries, volatile aroma compounds, such as terpenes, benzenoids, and phenylpropanoids, accumulate in exocarp and mesocarp tissues following the initiation of berry ripening $[38,82]$. Three transcripts (Figure 6C) encoding O-methyltransferases, which may participate in the biosynthesis of volatile compounds, were also detected [83]. The first Unigene (1608603_at, TC56956), which encodes a putative phloroglucinol O-methyltransferase, is involved in the biosynthesis of volatile 1,3,5-trimethoxybenzene, a compound not previously described in grape [83], displayed a very high transcript abundance at the beginning of berry development (E-L stage 31 ) before decreasing after véraison until E-L stage 36 and then increasing again in mature berries (Figure 6C). The second Unigene (1613542_at, TC62584) was expressed at E-L stage 31, but then declined. The third Unigene (1620469_at, CF209780) displayed very low transcript abundance with a slight increase following véraison (Figure 6C). Finally, two S-adenosyl-L-methionine (SAM):salicylic acid carboxyl methyltransferases were identified with developmentally-induced expression patterns. The first Unigene (1616348_at, TC52353) showed a broad peak of expression between E-L stages 32 to 35, whereas the second Unigene (1612552_at, TC57170) showed increased transcript abundance after véraison (E-L stage 34) (Figure 6C). Such genes are thought to play important roles in scent production or plant defense [84]. Little correlation between the level of sequence similarity and the structural similarity of their substrates has been observed for most of these protein families, so that gene functions have to be assigned following detailed biochemical testing [85].

\section{Organic acid and proline metabolism}

The acid:sugar balance at harvest is an important factor of wine quality as it affects important sensory attributes [15]. Two major organic acids that contribute to titratable acidity, tartrate and malate, are the most abundant organic acids in grapes and reach maximal concentrations around the end of Phase I (E-L stage 32; see Table 5). Tartrate concentrations were found to peak at E-L stage 32 and then declined steadily until harvest, E-L stage 38 (Figure 7A). Tartrate concentrations decreased in parallel with three different transcripts encoding L-idonate dehydrogenase (1622252_at, TC52651; 1613165_s_at, TC52651; 1612918_at, TC52651), a key enzyme in tartrate biosynthesis [86]. The innermost region of the berry pulp surrounding the seed has been shown to contain the highest tartrate concentrations [87]. Consistent with this observation, tartrate synthase transcripts have been shown to be more abundant in seeds than in outer mesocarp and skin tissues [71].

Like tartrate, malate concentrations peaked around E-L stage 32 , but then declined more rapidly than tartrate during berry ripening (Figure 7B). In contrast to the good correlation between tartrate and L-idonate dehydrogenase transcript abundance, there is a less obvious correlation between malate concentrations and the transcript abundance of Unigenes encoding malate dehydrogenases (Figure 7B). Transcript abundance for two isogenes encoding cytosolic NAD-dependent malate dehydrogenases (1612546_at, TC68207; 1609147_at, TC55437), which catalyze the interconversion of malate to oxaloacetate, increased during ripening. Transcripts for mitochondrial isoforms of the enzyme (1622059_at, TC60439; 1617448_at, TC54982) also increased over this same time period. In contrast, the transcript abundance of a NADPdependent malic enzyme (1609345_s_at, TC57092), which catalyzes the oxidative decarboxylation of malate to pyruvate, declined slightly from E-L stages 34 to 36, but then increased by stage 38 (Figure 7B). The slight increase in the expression of all of these enzymes together may contribute to the declining concentrations of malate during ripening. Very little is known about the mechanisms of malate transport processes in the phloem/xylem and within developing grape berries. The regulation of malate concentrations in berries appears to be quite complex. More research is needed to elucidate this well known developmental process.

Mature berries contain unusually high concentrations of free proline; proline being the most abundant amino acid in Cabernet Sauvignon [88,89]. Proline concentrations increased significantly at véraison and remained high until berries were fully ripe (Figure 7C). Transcripts encoding pyrroline-5-carboxylate synthetase (1619565_at, TC52705), the key regulatory enzyme in 

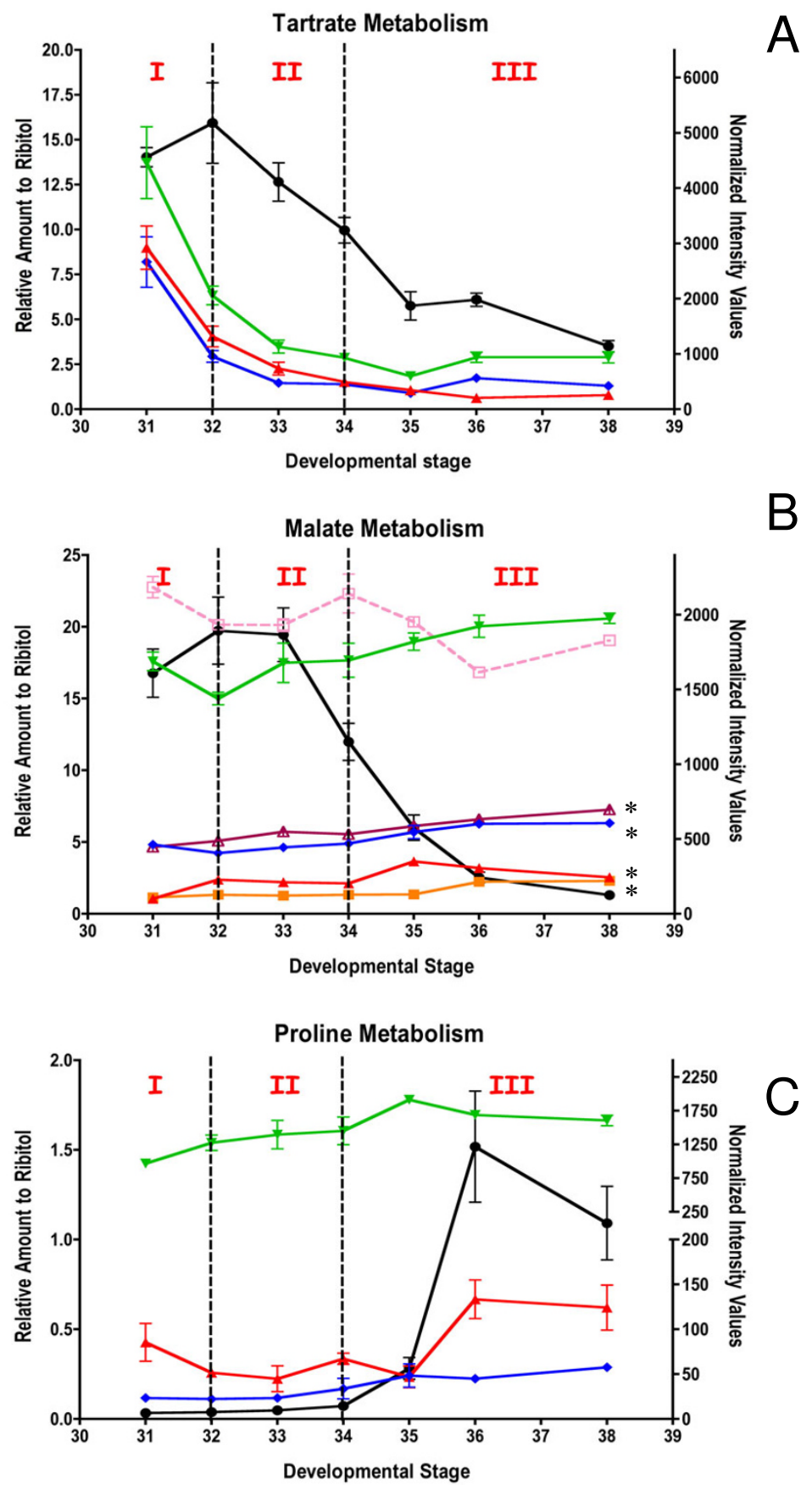

Figure 7

Organic acids and amino acids: metabolites and transcripts. A) Black solid round-tartrate, red solid triangle (1622252_at, TC5265I)-L-idonate dehydrogenase, green solid triangle (16I3 I65_s_at, TC5265I)-L-idonate dehydrogenase, blue solid diamond (1612918 at, TC5265I)-L-idonate dehydrogenase. B) Black solid round-malate, red solid triangle (16I2546_at, TC68207)-cytosolic MDH, green solid triangle (1609|47_at, TC55437)-cytosolic MDH, blue solid diamond (I622059_at, TC69439)-mitochondrial malate dehydrogenase (MDH), orange solid square (I6I7448_at, TC54982)-mitochondrial MDH, lavender open square (1609345_s_at, TC57092)-malic enzyme. C) Black solid round-proline, red solid triangle (1619565_at, TC52705)-pyrroline-5-carboxylate synthetase, green solid triangle (16I7293_s_at, BQ792635)-proline dehydrogenase, blue solid diamond (1610800_at, CK906448)-proline transporter. *: Transcripts that do not pass the two-fold ratio. All compounds amounts were normalized by a ribitol standard $(25 \mathrm{mg} / \mathrm{L})$. 
Table 5: Transcripts (TFR pool) related to organic and phenolic acid metabolism

\begin{tabular}{|c|c|c|c|c|c|c|c|}
\hline Probeset ID & $\begin{array}{l}\text { GenBank } \\
\text { Annotation }\end{array}$ & VvGI5 & UniProt ID & Gene Name Description & Function & Profile & Fold Change \\
\hline 1608526_at & CB974I98 & TC663I4 & Q5NBP4 & AOBP-like protein & Organic Acid & 11 & 6.48 \\
\hline 1618209_at & CF37302I & TC56I27 & P8228I & Ascorbate peroxidase & Organic acid & 3 & 3.59 \\
\hline 1617448_at & BQ795936 & TC54982 & Q9M6B3 & Malate dehydrogenase & Organic Acid & 10 & 3.48 \\
\hline 1606935_at & CB96953I & TC66898 & Q9SAK4 & Succinic semialdehyde dehydrogenase I & Organic acid & 3 & 3.05 \\
\hline 162064I_at & CF5II42I & TC52472 & Q39540 & AOBP-like protein & Organic Acid & 9 & 2.11 \\
\hline 1611871_at & CF4I5063 & TC54I32 & Q84UH4 & Dehydroascorbate reductase & Organic Acid & 19 & 2.1 \\
\hline |609|47_at & CB979I50 & TC55437 & Q645N0 & Malate dehydrogenase (cytosolic) & Organic Acid & 11 & 2.01 \\
\hline 1607417_at & CF5I 2464 & TC53733 & Q8L7U8 & Cinnamyl-alcohol dehydrogenase CADI & Phenolic Acid & 2 & 80.03 \\
\hline 1614643_at & CF2I4966 & TC5I729 & Q43237 & Caffeoyl-CoA O-Methyltransferase & Phenolic Acid & 2 & 34.34 \\
\hline 1611265_at & CF513719 & TC51900 & Q49LX7 & 4-coumarate:CoA ligase & Phenolic Acid & 11 & 25.11 \\
\hline 1620342_at & CF207053 & TC64352 & Q00763 & Caffeic acid 3-O-methyltransferase I & Phenolic Acid & 11 & 18.69 \\
\hline 1610935_at & CF404728 & TC6448I & Q75W19 & Ferulate-5-hydroxylase (FAHI) & Phenolic Acid & 2 & 13.64 \\
\hline 1619682_x_at & CF205002 & TC62835 & Q9M560 & Caffeic acid O-Methyltransferase & Phenolic Acid & 2 & 13.58 \\
\hline 1616434_s_at & AF239740 & TC62835 & Q9M560 & O-methyltransferase & Phenolic Acid & 2 & 11.53 \\
\hline 1609307_at & CD7I58I8 & TC66040 & $\mathrm{O} 24145$ & 4-coumarate--CoA ligase (At4CLI) & Phenolic Acid & 2 & 10.6 \\
\hline 1619450_s_at & CF215109 & TC52364 & Q00763 & O-methyltransferase & Phenolic Acid & 2 & 10.28 \\
\hline 1607475_s_at & CD0I2393 & TC64352 & Q3SCM5 & Caffeic acid O-methyltransferase & Phenolic Acid & 11 & 9.32 \\
\hline 1614423_at & CF5I7687 & TC668I5 & Q6DMZ8 & Cinnamoyl CoA Reductase & Phenolic Acid & 2 & 8.61 \\
\hline 1620650_s_at & CF207485 & TC69704 & Q9ATWI & Cinnamyl-alcohol dehydrogenase & Phenolic Acid & 1 & 5.95 \\
\hline 1616191_s_at & CB97I06I & TC707I5 & Q3HM04 & Cinnamate-4-Hydroxylase & Phenolic Acid & 3 & 5.78 \\
\hline 1613542_at & CF209028 & TC62584 & Q7X9J0 & O-methyltransferase & Phenolic Acid & 2 & 5.54 \\
\hline 1622267_at & CF516149 & TC64537 & 065152 & Cinnamyl-alcohol dehydrogenase & Phenolic Acid & 3 & 4.48 \\
\hline 1619320_at & CB974305 & TC66743 & P31687 & 4-coumarate:CoA ligase 3 (4CL3) & Phenolic Acid & 3 & 4.21 \\
\hline 1619808_at & СВ972340 & TC54722 & 065152 & Cinnamyl-alcohol dehydrogenase & Phenolic Acid & 3 & 3.96 \\
\hline 1611249_s_at & CF5I7I55 & TC5I769 & 065152 & Cinnamyl-alcohol dehydrogenase & Phenolic Acid & 3 & 3.93 \\
\hline |61383|_at & CD801016 & TC58955 & Q516D6 & Sinapyl alcohol dehydrogenase & Phenolic Acid & 3 & 3.36 \\
\hline 1613548_at & CB009193 & TC68990 & Q8H8C9 & 4-coumarate:CoA ligase & Phenolic Acid & 11 & 3.19 \\
\hline 1615439_at & CF2I 3244 & TC63II2 & P30359 & Cinnamyl alcohol dehydrogenase 2 & Phenolic Acid & 2 & 2.38 \\
\hline 1609327_at & CF208599 & TC68572 & AIYIQ2 & Cinnamyl-alcohol dehydrogenase I & Phenolic Acid & 2 & 2.33 \\
\hline 1607|63_at & CF4I5I7I & - & Q8LSQ3 & 4-coumarate:CoA ligase & Phenolic Acid & 3 & 2.22 \\
\hline |6135|I_at & BQ796246 & TC59682 & Q65CJ7 & Hydroxyphenylpyruvate reductase & Phenolic Acid & 11 & 2.22 \\
\hline 1616445_at & CD7I60I4 & TC57545 & Q9LYJ0 & Cinnamoyl CoA Reductase & Phenolic Acid & 16 & 2.11 \\
\hline
\end{tabular}

proline biosynthesis, remained relatively constant with a small peak of expression occurring at E-L stage 35 (Figure 7C). Proline dehydrogenase transcripts (1617293_s_at, BQ792635), which encode the first enzymatic step in proline catabolism, increased only during the latter stages of berry development. These mRNA expression patterns are consistent with earlier reports and with protein expression patterns of these enzymes [88]. Proline accumulation correlated poorly with steady-state transcript and protein abundance changes for these two enzymes indicating that proline production is regulated by posttranslational mechanisms [88]. Steady-state transcripts encoding a proline transport protein (1610800_at, CK906448) also increased in conjunction with proline abundance.

\section{Sugar metabolism}

Sugar accumulation in grape berries has been well studied because sugar content is a key factor in producing wine. In contrast to organic acids, hexose sugars (i.e., Glc and Fru) begin to accumulate substantially in the lag phase (Phase II) and continue thereafter. In grapevines, carbohydrates produced during photosynthesis are exported from the leaf as sucrose and transported in the phloem to the berry cluster $[90,91]$. Prior to véraison, most sugars imported into the berries are metabolized with little if any storage of these compounds. Following véraison, however, sugars accumulate in the vacuole to high levels in the form of glucose and fructose following the enzymatic cleavage of sucrose (mainly in the apoplast, but also in the cytoplasm and vacuole). Monosaccharide transporters direct the transport of these sugars through different organelles [92].

In the berries in this study, fructose was more abundant than glucose; in contrast sucrose concentrations remained relatively low and constant throughout berry development (Figure 8A). Transcript abundance for the Unigene encoding sucrose synthase (1609402_at, TC62599), increased gradually over berry development consistent with increased hexose accumulation in the berry. This Unigene has high homology with the sucrose synthase (CiTSUSA) in Citrus unshiu [93]. CiTSUSA also increases with fruit development and catalyzes the reaction in the 

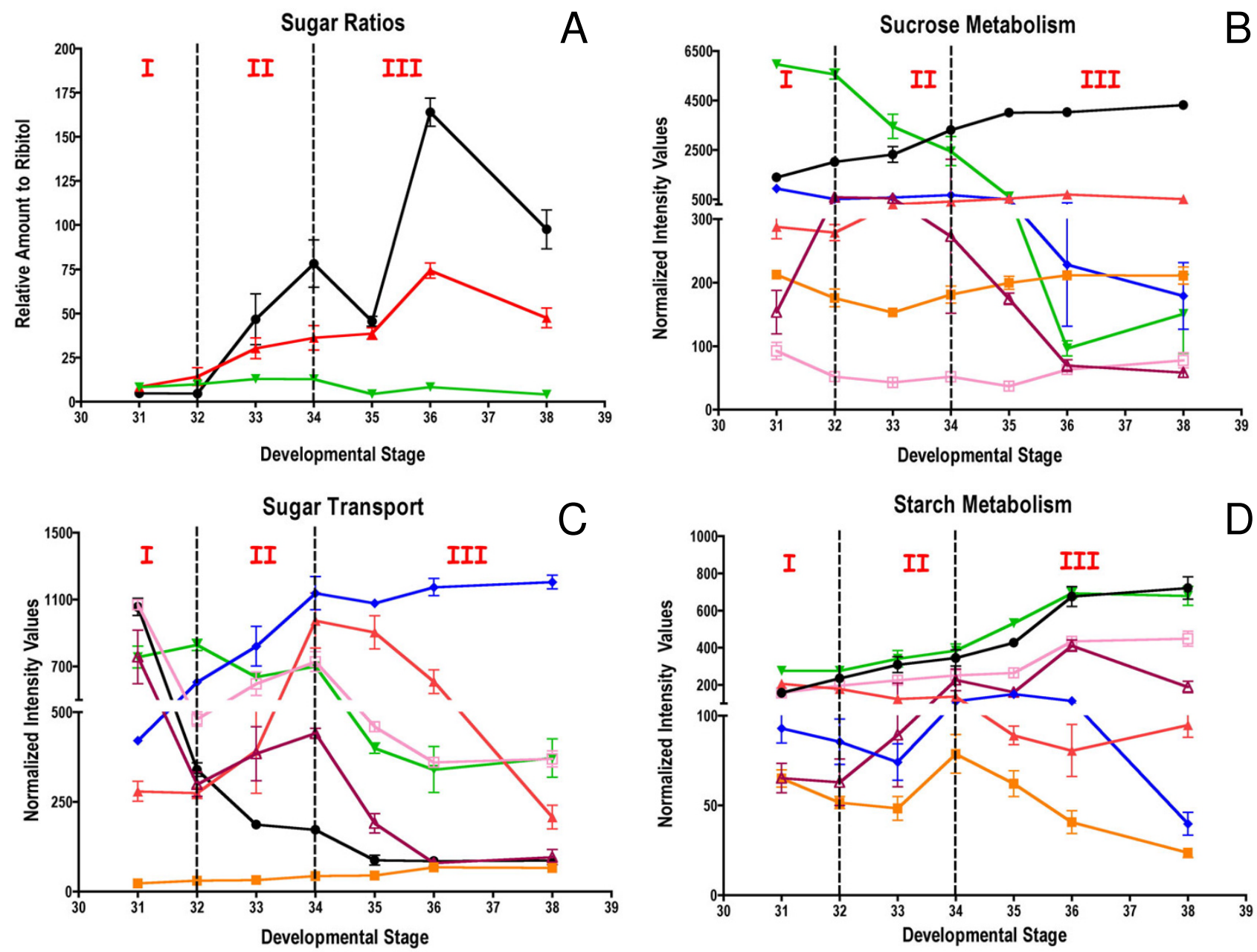

Figure 8

Hexose sugars, transporters, and starch: metabolites and transcripts. A) Black solid round-fructose, red solid triangle-glucose, green solid triangle-sucrose. B) Black solid round (1609402_at, TC62599)-sucrose synthase, red solid triangle (I608257_at, TC68 I35)-sucrose-6-phosphate phosphatase, green solid triangle (I6I I6I3_at, TC60693)-invertase (GINI), blue solid diamond (1612836_at, TC57719)-invertase (GIN2), orange solid square (1620628_at, TC67908)-neutral invertase, lavender open square (161 1027 at, TC56057)-acidic invertase, brown open triangle (1616255_at, TC57339)-fructokinase. C) Black solid round (1616083_at, TC5 I694)-VvHTI (hexose transporter I), red solid triangle (I6I 5257_at, TC65400)-VvHT6 (hexose transporter 6), green solid triangle (1615697_at, TC5 I724)-VvSUC27 (sucrose transporter), blue solid diamond (I60899I_at, TC60060)-plastidial glucose transporter, orange solid square (1613408_at, TC66667)-polyol transporter, lavender open square (1619379_at, TC5880 I)-plastidial triose phosphate transporter, brown open triangle (1622I57_at, TC6I733)-plastidial triose phosphate transporter. D) Black solid round (I6I557I_at, TC5355I)-starch synthase, red solid triangle (I6I360I_at, TC67353)-starch synthase, green solid triangle (I617068_at, TC5462I)-plastidial alpha-glucan, water dikinase, blue solid diamond (16I794I_at, TC62494)-plastidial alpha-glucan, water dikinase, orange solid square (I622I20_at, TC54533)-starch phosphorylase, lavender open square (1613 I88_at, TC70258)- $\alpha$-amylase, brown open triangle (I617124_at, TC67979)- $\beta$-amylase. All compounds amounts were normalized by a ribitol standard ( $25 \mathrm{mg} / \mathrm{L})$.

cleavage direction (sucrose to UDP-glucose and fructose). Komatsu et al. [93] suggest that the action of this gene may be important for sink strength.

Sucrose-6-phosphate phosphohydrolase (1608257_at, TC68135), which catalyzes the last step in sucrose synthesis, showed a slight increase in transcript abundance after E-L stage 32 and then remained relatively constant throughout the remainder of berry development (Figure 8B). In contrast, the transcript abundance of two vacuolar invertases, GIN1 and GIN2 (1611613_at, TC60693; 1612836_at, TC57719), which catalyze the 
catabolism of sucrose to fructose and glucose, declined over the course of berry development (Figure 8B), consistent with an earlier report [94]. The mRNA expression of these two invertases is consistent with the early increases in sugar accumulation during Phase II (E-L stages 32 to $34)$. On the other hand, transcript abundance for a neutral invertase (1620628_at, TC67908) and a cell wall acid invertase (1611027_at, TC56057) remained relatively constant during the course of berry development consistent with earlier reports on the amount and activity of these enzymes in developing berries [95]. In grape berries, sucrose cleavage is largely catalyzed by cell wall bound invertases [95]. Sucrose cleavage is usually associated with cell wall invertase activity at the onset of ripening, together with a shift towards apoplastic phloem unloading of sugars in berries during this same period of time [95]. Finally, transcripts encoding fructokinase (1616255_at, TC57339), which catalyzes the formation of fructose-6-phosphate and may regulate starch formation, declined in abundance in a similar manner as GIN1 and GIN2 following a peak of expression at E-L stage 32 .

In most sink cells, sucrose is either cleaved by invertase into glucose and fructose or degraded by sucrose synthase into uridine-5'-diphosphate (UDP) glucose and fructose for subsequent metabolism and biosynthesis $[96,97]$. Cell wall invertases appear to play the main role in the cleavage of sucrose during Phase III of berry development [95]. However, the increase in sucrose synthase during Phase III of berry development indicates that this isogene may participate in the catabolism of sucrose to fructose and glucose. Alternatively, this sucrose synthase isogene may play a critical role in cellulose synthesis associated with Phase III cell expansion similar to its role in cotton fiber elongation [98]. Two cellulose synthase isogenes (1607069_at, TC53461; 1611149_at, TC56091) displayed increased transcript abundance during Phase II and III, consistent with this hypothesis (see Table 1). Additional developmentally regulated transcripts related to carbohydrate metabolism and transport are summarized in Table 6.

\section{Hexose and triose phosphate transport}

The transcript abundances of numerous hexose and triosephosphate transporters varied considerably over the course of berry development (Figure 8C) indicating that each may fulfill different transport roles. The transcript abundance for a VvHT1 (1616083_at, TC51694), a previously described hexose transporter (VvHT1) located at the sieve cell-companion cell interface in the phloem and thought to play a major role in providing energy (mainly from glucose) for cell division and cell growth during the early stages of berry development [99], was high during Phase I, but then declined rapidly during ripening; this is largely consistent with an earlier report [18]. A second hexose transporter, VvHT6 (1615257_at; TC65400) exhibited a peak in transcript abundance near the start of véraison (E-L stage 34), which correlated well with hexose accumulation in the berries (Figure $8 \mathrm{~A}$ ), indicating that this transporter may play a significant role in hexose accumulation during berry ripening. Another previously described sucrose transporter (VvSUC27; 1615697_at, TC51724) [100], exhibited decreased transcript abundance throughout berry development consistent with earlier observations.

A putative plastidic glucose transporter (1608991_at, TC60060) showed increased transcript abundance up to E-L stage 34 and then remained constant throughout berry ripening (Figure $8 \mathrm{C}$ ). The transcript abundance of a putative plasma membrane sugar/polyol transporter (1613408_at, TC66667), which resembles the AtPLT5 gene from A. thaliana [101] and is also capable of hexose transport, increased gradually over the course of berry development. In addition, two transcripts encoding a plastidial phosphate translocator-like (PTL) protein (1619379_at, TC58801) and a plastidial triosephosphate/ phosphate translocator, TPT (1622157_at, TC61733) [102] displayed similar expression patterns that peaked at E-L stage 34 and then declined with berry ripening. The observed patterns of expression of the plastidial glucose and triosephosphate transporters indicate that both glucose and triosephosphates may be mobilized as export products as a result of active starch metabolism in plastids of developing and ripening berries.

Finally, a sorbitol transporter (Figure 9) that has high homology with a cherry sorbitol transporter (PcSOT2) [103], has high transcript abundance early in fruit development as it does in cherry fruit. This transporter has high specificity for sorbitol as compared to its isomer, mannitol [103]. We were able to detect a sugar alcohol in our polar extracts using GC-MS, but were unable to distinguish whether it was sorbitol or mannitol. Further work will be done to distinguish sorbitol from mannitol. Note, however, that sorbitol has been detected in the sap of grapevines [104].

\section{Starch metabolism}

Starch metabolism in developing and ripening grape berries is poorly understood. Starch synthase I catalyzes the elongation of glucans by the addition of glucose residues from ADP-glucose through the formation of $\alpha-1,4$ linkages and is a major determinant for the synthesis of transient starch reserves in plants [105]. Our data indicate that starch metabolism is significant in berries. Starch concentrations declined significantly during Phase III of berry development; E-L stage 35, 36 and 38 were equal to $774 \pm$ $57,715 \pm 54$ and $554 \pm 28 \mu \mathrm{g}$ of glucose per $\mathrm{g}$ fresh weight of berry, respectively (mean $\pm \mathrm{SE}$ ). 
Table 6: Transcripts (TFR pool) related to carbohydrate metabolism and transport categorized by the first hit in the MIPS2 catalog

\begin{tabular}{|c|c|c|c|c|c|c|c|}
\hline Probeset ID & $\begin{array}{l}\text { GenBank } \\
\text { Annotation }\end{array}$ & VvGI5 & UniProt ID & Gene Name Description & Function & Profile & Fold Change \\
\hline 161806I_a_at & CF5I4699 & TC52548 & O78327 & Transketolase & Amino Acids Metabolism & 20 & 3.44 \\
\hline 1614105_at & CB968800 & TC70460 & Q4JIY3 & Pyruvate dehydrogenase & Amino Acids Metabolism & 11 & 2.2 \\
\hline 1616700_at & CB910092 & TC53526 & Q9SLY2 & Sucrose synthase & Carbohydrate metabolism & 3 & 227.67 \\
\hline 1611613_at & BQ79677I & TC60693 & Q9S944 & Invertase & Carbohydrate metabolism & 3 & 61.86 \\
\hline 1622656_at & CF2I 5745 & TC61716 & Q5NA70 & Glucan endo-I,3-b-glucosidase & Carbohydrate metabolism & 2 & 53.85 \\
\hline 1614716_at & CB978853 & TC58640 & Q6Z8F4 & Phosphoribulokinase & Carbohydrate metabolism & 12 & 51.76 \\
\hline 1617719_at & CB975632 & TC553I4 & Q6IV07 & $\begin{array}{l}\text { UDP-glucose:protein } \\
\text { transglucosylase }\end{array}$ & Carbohydrate metabolism & 2 & 40.93 \\
\hline 1607442_at & CF4037I7 & - & Q50HW0 & Glucuronosyltransferase & Carbohydrate metabolism & 2 & 38.54 \\
\hline 1622115_at & CD004218 & TC60627 & Q9SRX8 & b-glucosidase & Carbohydrate metabolism & 10 & 26.34 \\
\hline 1611970_at & CF207II95 & TC62847 & Q9LKY6 & Glucose acyltransferase & Carbohydrate metabolism & 3 & 25.74 \\
\hline 1620679_at & CB972076 & TC5335I & Q9LV33 & b-glucosidase & Carbohydrate metabolism & 14 & 16.26 \\
\hline 1621352_at & BQ794457 & TC59789 & Q8GT4I & Invertase inhibitor & Carbohydrate metabolism & 11 & 16.06 \\
\hline 1608932_at & CB982469 & TC6320I & Q59J80 & Glucosyltransferase & Carbohydrate metabolism & 11 & 15.38 \\
\hline 1620347_at & CA8I4065 & TC66065 & Q5QPZ6 & Glycosyltransferase & Carbohydrate metabolism & 10 & 15.36 \\
\hline 1622282_at & $\mathrm{CD} 7 \mid 2313$ & TC54393 & Q7XAE2 & Fructokinase & Carbohydrate metabolism & 2 & 13.54 \\
\hline 1616642_at & BQ80022I & TC64250 & Q9FEP9 & Glycerol-3-phosphate acyltransferase & Carbohydrate metabolism & 16 & 11.07 \\
\hline 1616255_at & CF5I6475 & TC57339 & 082616 & Fructokinase & Carbohydrate metabolism & 12 & 10.13 \\
\hline 1612918_at & СB972844 & TC5265I & Q9MBD7 & $\begin{array}{l}\text { NAD-dependent sorbitol } \\
\text { dehydrogenase }\end{array}$ & Carbohydrate metabolism & 2 & 9.14 \\
\hline 1608393_at & CF403620 & TC64860 & O22658 & ADP-glucose pyrophosphorylase & Carbohydrate metabolism & 7 & 8.79 \\
\hline 1621067_at & CF5II425 & TC51908 & Q8W3C8 & Glucose acyltransferase & Carbohydrate metabolism & 3 & 8.2 \\
\hline 1612883_at & CB911656 & TC60606 & O22060 & Sucrose-phosphate synthase I & Carbohydrate metabolism & 16 & 7.92 \\
\hline 1617035_s_at & CF205538 & TC64995 & Q9XGN4 & Galactinol synthase & Carbohydrate metabolism & 11 & 7.73 \\
\hline 1609652_s_at & CF2I5703 & TC59328 & Q9FNI7 & Glucosyltransferase & Carbohydrate metabolism & 3 & 7.5 \\
\hline 1617309_at & CB922444 & TC59505 & Q8LFT7 & Aldehyde dehydrogenase & Carbohydrate metabolism & 10 & 7.26 \\
\hline 1619190_at & CD720196 & TC54797 & Q6H5W0 & Alcohol dehydrogenase & Carbohydrate metabolism & 3 & 6.66 \\
\hline 1616107_s_at & CD7I5446 & TC67979 & Q94EU9 & b-amylase & Carbohydrate metabolism & 9 & 6.34 \\
\hline 1618409_at & CF5I4784 & TC52918 & Q94G86 & Glucan endo-I,3-b-glucosidase & Carbohydrate metabolism & 3 & 6.26 \\
\hline 1620624_at & CB969436 & TC52478 & Q94IP3 & UDP-Glucose Transferase & Carbohydrate metabolism & 2 & 6.21 \\
\hline 1611680_at & CF4I549I & TC58448 & Q50HU7 & Glycosyltransferase & Carbohydrate metabolism & 2 & 5.8 \\
\hline 1612465_at & CF568806 & TC53602 & 065736 & b-galactosidase & Carbohydrate metabolism & 4 & 5.76 \\
\hline 161807I_at & CF518536 & TC5438I & Q9M8Y0 & O-linked GlcNAc transferase & Carbohydrate metabolism & 16 & 5.7 \\
\hline 1611804_at & CF513259 & TC62252 & Q9ZVX4 & Glucose acyltransferase & Carbohydrate metabolism & 12 & 5.52 \\
\hline 1617454_at & BQ798893 & - & Q8VYG2 & Galactokinase & Carbohydrate metabolism & 2 & 5.43 \\
\hline 1612836_at & CF403299 & TC57719 & Q9S943 & Invertase & Carbohydrate metabolism & 3 & 5.28 \\
\hline 1618517_at & CB97I 627 & TC53602 & Q93X58 & b-galactosidase & Carbohydrate metabolism & 4 & 5.01 \\
\hline 1622074_at & BQ794083 & - & Q84JP7 & $\begin{array}{l}\text { Phosphoenolpyruvate carboxylase } \\
\text { kinase }\end{array}$ & Carbohydrate metabolism & 12 & 4.66 \\
\hline 161557|_at & CB983I56 & TC5355I & Q9FNF2 & Starch synthase & Carbohydrate metabolism & 9 & 4.58 \\
\hline 1622543_at & CB977855 & TC61696 & Q84V96 & Aldehyde dehydrogenase & Carbohydrate metabolism & I & 4.57 \\
\hline 1610724_at & CB916342 & TC6365I & Q652SI & $\begin{array}{l}\text { Fructose/tagatose bisphosphate } \\
\text { aldolase }\end{array}$ & Carbohydrate metabolism & 11 & 4.57 \\
\hline 1620997_at & CD799067 & TC63159 & Q84LII & Galactose dehydrogenase & Carbohydrate metabolism & 2 & 4.55 \\
\hline 1619223_s_at & CB005867 & TC52910 & Q9SLS2 & Sucrose synthase & Carbohydrate metabolism & 2 & 4.46 \\
\hline 1615614_at & CF405918 & TC54I97 & Q9M310 & Glucosyltransferase & Carbohydrate metabolism & 2 & 4.28 \\
\hline 1622065_at & CD8017I4 & - & Q94FA7 & Fructose-bisphosphatase & Carbohydrate metabolism & 3 & 4.17 \\
\hline 1622503_at & CF203022 & TC69704 & Q9ATWI & Mannitol dehydrogenase & Carbohydrate metabolism & 1 & 4.16 \\
\hline 1615634_at & CB970085 & TC69016 & Q8L9U9 & Glucose acyltransferase & Carbohydrate metabolism & 12 & 3.86 \\
\hline 1607324_at & CD7I9348 & TC54773 & P94078 & a-mannosidase & Carbohydrate metabolism & 2 & 3.85 \\
\hline $1611112 \_$at & CB97। 308 & TC5I885 & Q7XPW5 & Phosphomannomutase & Carbohydrate metabolism & 3 & 3.85 \\
\hline 1616325_at & CF2II8I5 & TC53040 & Q6Q2Z9 & Phosphoenolpyruvate carboxylase & Carbohydrate metabolism & 3 & 3.84 \\
\hline 1617068_at & CF519166 & TC5462I & Q9SGX4 & Water dikinase & Carbohydrate metabolism & 18 & 3.8 \\
\hline 1611604_at & CB916873 & TC5485I & Q8LPJ3 & a-mannosidase & Carbohydrate metabolism & 3 & 3.78 \\
\hline 1620724_at & CB915307 & TC66445 & 048628 & Phosphofructo-I-kinase & Carbohydrate metabolism & 11 & 3.73 \\
\hline 1616500_at & AFI94I75 & TC52882 & Q9FZ00 & Alcohol dehydrogenase & Carbohydrate metabolism & 10 & 3.69 \\
\hline 1612870_s_at & CF20I540 & TC66152 & QODAH4 & $\begin{array}{l}\text { GDP-4-keto-6-deoxy-D-mannose- } \\
\text { 3,5-epimerase-4-reductase }\end{array}$ & Carbohydrate metabolism & 3 & 3.66 \\
\hline 1608527_at & CF5I5950 & TC58983 & Q9FJ95 & Sorbitol dehydrogenase & Carbohydrate metabolism & 10 & 3.6 \\
\hline
\end{tabular}


Table 6: Transcripts (TFR pool) related to carbohydrate metabolism and transport categorized by the first hit in the MIPS2 catalog

\begin{tabular}{|c|c|c|c|c|c|c|c|}
\hline 1619457_at & CB96973I & TC63406 & P93653 & Trehalose-6-phosphate synthase & Carbohydrate metabolism & 12 & 3.58 \\
\hline 1611154_at & CF204490 & - & Q42954 & Pyruvate kinase & Carbohydrate metabolism & 3 & 3.54 \\
\hline 1614552_at & CB978862 & TC54I60 & Q5SMZI & Aldose I-epimerase & Carbohydrate metabolism & 12 & 3.52 \\
\hline 1608263_a_at & BQ794795 & TC5I76I & Q9M6B4 & Alcohol dehydrogenase & Carbohydrate metabolism & II & 3.5 \\
\hline 1617186_at & CF4I5580 & TC70II9 & O65856 & Glucose-6-phosphate dehydrogenase & Carbohydrate metabolism & I & 3.49 \\
\hline 1608907_s_at & CA809004 & TC517I3 & Q9XGN4 & Galactinol synthase & Carbohydrate metabolism & 11 & 3.4 \\
\hline 1613182_at & CB982869 & - & Q6PP98 & Pyruvate dehydrogenase kinase & Carbohydrate metabolism & 11 & 3.37 \\
\hline 1612414_at & CD7I5284 & TC5860I & Q42910 & Pyruvate phosphate dikinase & Carbohydrate metabolism & 10 & 3.34 \\
\hline 1622120_at & CF519014 & TC54533 & P27598 & Starch phosphorylase & Carbohydrate metabolism & 21 & 3.33 \\
\hline 1606536_at & CB97I452 & - & Q8S9A7 & Glucosyltransferase & Carbohydrate metabolism & 3 & 3.29 \\
\hline 1622606_at & CB910226 & TC52786 & Q6DW08 & GDP-mannose pyrophosphorylase & Carbohydrate metabolism & 3 & 3.28 \\
\hline 1610766_at & CF2I 2685 & TC5329I & Q7YI52 & Galactokinase & Carbohydrate metabolism & 11 & 3.24 \\
\hline 1615270_at & CF208284 & TC70917 & Q6K963 & Callose synthase & Carbohydrate metabolism & 21 & 3.23 \\
\hline 1615167_at & CF519116 & TC65652 & Q9LFQ0 & Glycosylation enzyme & Carbohydrate metabolism & 12 & 3.2 \\
\hline 1606774_at & CF4I5I65 & TC7026I & Q8L7J4 & Pyruvate kinase & Carbohydrate metabolism & II & 3.14 \\
\hline 1609402_at & BQ794844 & TC62599 & Q9SLY2 & Sucrose synthase & Carbohydrate metabolism & 11 & 3.09 \\
\hline 1608100_at & CF4040I3 & TC51810 & Q8S569 & Phosphoenolpyruvate carboxylase & Carbohydrate metabolism & 2 & 3.07 \\
\hline 1609470_at & CF203556 & - & Q8LFZ9 & Sucrase & Carbohydrate metabolism & 5 & 3.04 \\
\hline 1614023_at & CF4I4667 & - & P46275 & Fructose-I,6-bisphosphatase & Carbohydrate metabolism & 2 & 3.01 \\
\hline 1618726_at & CF21II03 & TC60540 & Q5JNJI & $\begin{array}{l}\text { Trehalose-6-phosphate synthase/ } \\
\text { phosphatase }\end{array}$ & Carbohydrate metabolism & 4 & 3 \\
\hline 1614982_at & CF2II066 & TC61602 & Q9C9P3 & GDP-mannose pyrophosphorylase & Carbohydrate metabolism & 3 & 2.99 \\
\hline 1616783_at & CF405837 & TC58450 & P93344 & Aldehyde dehydrogenase & Carbohydrate metabolism & II & 2.95 \\
\hline 1616630_at & CF603093 & TC56347 & Q94LX9 & Phosphoenolpyruvate carboxylase & Carbohydrate metabolism & 16 & 2.95 \\
\hline 1620905_at & CF215819 & TC68052 & Q6RK07 & UDP-glucose dehydrogenase & Carbohydrate metabolism & 21 & 2.89 \\
\hline |62186|_at & CF209183 & TC65564 & Q94AS2 & b-amylase & Carbohydrate metabolism & 11 & 2.87 \\
\hline 1613188_at & CA817889 & TC70258 & Q5BLYI & a-amylase & Carbohydrate metabolism & II & 2.85 \\
\hline 1608207_at & CB343787 & TC63660 & Q84V96 & Aldehyde dehydrogenase & Carbohydrate metabolism & 3 & 2.7 \\
\hline 1611808_at & CF205006 & TC67979 & Q94EU9 & b-amylase & Carbohydrate metabolism & 9 & 2.69 \\
\hline 1610410_at & CB342966 & TC61245 & O64733 & Glucosyltransferase & Carbohydrate metabolism & 9 & 2.67 \\
\hline 161185I_at & BQ799617 & TC52022 & Q9FIK0 & Phosphofructo-I-kinase & Carbohydrate metabolism & 10 & 2.67 \\
\hline 1609545_at & CF5I4819 & TC52560 & Q4R0T9 & ADP-sugar diphosphatase & Carbohydrate metabolism & II & 2.64 \\
\hline 1617368_at & CF5I 2540 & - & $\mathrm{E}|3| 3$ & Glucan endo-I,3-b-glucosidase & Carbohydrate metabolism & 3 & 2.63 \\
\hline 1615623_at & CF5II8I3 & TC55899 & 064733 & Glucose acyltransferase & Carbohydrate metabolism & 2 & 2.63 \\
\hline 1620375_at & CA8I4054 & TC62I55 & Q8LK43 & $\begin{array}{l}\text { Glycogene synthase kinase-like } \\
\text { kinase }\end{array}$ & Carbohydrate metabolism & 7 & 2.58 \\
\hline 161360I_at & CB978458 & TC67353 & O64927 & Starch synthase & Carbohydrate metabolism & 3 & 2.56 \\
\hline 1618125_at & BQ798742 & - & Q94KE3 & Pyruvate kinase & Carbohydrate metabolism & 16 & 2.52 \\
\hline 161794I_at & CB9|4224 & TC62494 & 081505 & Water dikinase & Carbohydrate metabolism & II & 2.52 \\
\hline 1621073_at & CB914439 & TC55380 & Q7XEL0 & GDP-mannose-3",5"-epimerase & Carbohydrate metabolism & 3 & $2.5 \mathrm{I}$ \\
\hline 1620165_at & CA817563 & TC560I4 & Q84YG5 & Isoamylase & Carbohydrate metabolism & II & 2.51 \\
\hline 1613060_at & CF2I4238 & TC53819 & Q9M3B6 & Pyruvate kinase & Carbohydrate metabolism & 18 & 2.51 \\
\hline 1620904_at & CF609568 & TC58209 & Q9SAD5 & $\begin{array}{l}\text { b-I,4-N- } \\
\text { acetylglucosaminyltransferase }\end{array}$ & Carbohydrate metabolism & 16 & 2.49 \\
\hline 1611027_at & CB978747 & TC56057 & Q3L7K5 & Invertase & Carbohydrate metabolism & 20 & 2.49 \\
\hline 1608995_at & BQ796616 & TC5494I & Q84NI6 & a-galactosidase & Carbohydrate metabolism & II & 2.48 \\
\hline 1622806_at & CВ009073 & TC63769 & Q6VWJ5 & Fructokinase & Carbohydrate metabolism & I & 2.48 \\
\hline 1609510_at & CF5I3342 & TC69905 & Q0WV85 & O-linked GlcNAc transferase & Carbohydrate metabolism & 16 & 2.47 \\
\hline 1609232_at & CA811215 & TC56883 & Q9ZVJ5 & Phosphoglucomutase & Carbohydrate metabolism & 15 & 2.45 \\
\hline 16135|4_s_at & CF202452 & TC5494I & Q9M442 & a-galactosidase II & Carbohydrate metabolism & 11 & 2.44 \\
\hline 1613025_at & CF403382 & TC69507 & Q9SNY3 & GDP-mannose 4,6 dehydratase I & Carbohydrate metabolism & 21 & 2.43 \\
\hline |6|45|4_at & CF40536I & TC66847 & Q84V39 & Glucan endo-I,3-b-glucosidase & Carbohydrate metabolism & 2 & 2.42 \\
\hline 1608156_at & CF207998 & TC58210 & Q9XEY7 & Trehalase & Carbohydrate metabolism & 11 & 2.4 \\
\hline 1612056_at & BQ795970 & - & Q5BMC5 & Phosphomannose isomerase & Carbohydrate metabolism & 19 & 2.39 \\
\hline 1612295_at & CF5I24I7 & TC67968 & Q5VMJ5 & $\begin{array}{l}\text { Pyrophosphate-dependent } \\
\text { phosphofructo-I-kinase }\end{array}$ & Carbohydrate metabolism & 15 & 2.38 \\
\hline 1609079_at & BQ796278 & TC60979 & Q94KE3 & Pyruvate kinase & Carbohydrate metabolism & 13 & 2.36 \\
\hline 1615874_at & CF403960 & TC54126 & Q93XR7 & $\begin{array}{l}\text { Fructose-6-phosphate,2-kinaseV } \\
\text { fructose-2,6-bisphosphatase }\end{array}$ & Carbohydrate metabolism & 2 & 2.35 \\
\hline 1608883_at & CA818676 & TC605I5 & Q94AA4 & $\begin{array}{l}\text { Pyrophosphate-dependent } \\
\text { phosphofructo-I-kinase }\end{array}$ & Carbohydrate metabolism & 15 & 2.34 \\
\hline 1616002_s_at & CB345569 & TC5226I & Q8LL68 & Aldolase & Carbohydrate metabolism & 3 & 2.29 \\
\hline 1620865_at & CB917214 & TC66899 & Q7XBE4 & Enolase & Carbohydrate metabolism & II & 2.29 \\
\hline
\end{tabular}


Table 6: Transcripts (TFR pool) related to carbohydrate metabolism and transport categorized by the first hit in the MIPS2 catalog

\begin{tabular}{|c|c|c|c|c|c|c|c|}
\hline 1607/47_at & CF404016 & - & Q5BLY0 & a-amylase & Carbohydrate metabolism & 10 & 2.28 \\
\hline 1607727_at & CB97632I & TC57680 & Q5IHI4 & Sucrose-6-phosphate phosphatase & Carbohydrate metabolism & 11 & 2.26 \\
\hline 1614707_at & BQ7993।3 & TC53692 & P328II & a-glucan phosphorylase & Carbohydrate metabolism & 21 & 2.25 \\
\hline 1610277_at & CF2080I6 & TC705I4 & Q50HW6 & b-I,3-glucuronosyltransferase & Carbohydrate metabolism & 2 & 2.25 \\
\hline 1621432 s_at & CD005042 & TC52007 & Q8VXZ7 & a-galactosidase & Carbohydrate metabolism & 10 & 2.18 \\
\hline 1621053_at & CF4|4284 & TC63955 & Q6VWJ5 & Fructokinase & Carbohydrate metabolism & 3 & 2.16 \\
\hline 1621719_at & CF404994 & TC65554 & Q8LGH6 & Dihydrolipoamide S-acetyltransferase & Carbohydrate metabolism & 3 & 2.14 \\
\hline 1619373_at & CB920390 & TC69024 & P80572 & Alcohol dehydrogenase & Carbohydrate metabolism & 3 & 2.13 \\
\hline 16146/2_at & CF5I3589 & TC63370 & Q9LSG3 & Glucose acyltransferase & Carbohydrate metabolism & 2 & 2.13 \\
\hline 1615252_at & BQ792622 & TC60550 & Q5N8HI & Hydrolase-like protein & Carbohydrate metabolism & 3 & 2.08 \\
\hline 1612568_at & CF405938 & TC67425 & Q9LIB2 & Glycogen phosphorylase B & Carbohydrate metabolism & 13 & 2.07 \\
\hline 1614153_at & CF207979 & TC5449I & Q7EYK9 & $\begin{array}{l}\text { Glucose-6-phosphate I- } \\
\text { dehydrogenase }\end{array}$ & Carbohydrate metabolism & 4 & 2.03 \\
\hline 1621378_at & BQ794342 & TC61809 & Q4258I & $\begin{array}{l}\text { Ribose-phosphate } \\
\text { pyrophosphokinase I }\end{array}$ & Nucleotide metabolism & II & 4.5 \\
\hline 1607578_at & CF4I55I9 & TC56533 & $\mathrm{O} 22141$ & Nucleotide sugar epimerase & Nucleotide metabolism & 2 & 4.09 \\
\hline 1608708_at & CF2II873 & TC53982 & Q9SU83 & Nucleotide pyrophosphatase & Nucleotide metabolism & 18 & 3.73 \\
\hline 1616669_at & CF209I74 & TC54382 & Q3EAE2 & dTDP-4-dehydrorhamnose reductase & Nucleotide metabolism & 3 & 3.45 \\
\hline 1609246_s_at & CF206363 & TC54199 & Q655Y8 & UDP-glucose 4-epimerase & Nucleotide metabolism & 4 & 3.14 \\
\hline 1607889_a_at & CB976234 & TC58106 & Q6IVK4 & UDP-glucuronate decarboxylase 2 & Nucleotide metabolism & 4 & 2.55 \\
\hline 1622819_at & BQ798887 & TC59368 & O2214I & Nucleotide sugar epimerase & Nucleotide metabolism & 2 & 2.52 \\
\hline 1616344_at & CF209I36 & TC68545 & Q6XP48 & UDP-glucose 4-epimerase & Nucleotide metabolism & 21 & 2.52 \\
\hline 1614498_at & CF213286 & TC57825 & O6578I & UDP-galactose 4-epimerase & Nucleotide metabolism & 21 & 2.32 \\
\hline 1620930_s_at & CF2I 2327 & TC5I843 & Q6IVK4 & UDP-glucuronate decarboxylase 2 & Nucleotide metabolism & 4 & 2.21 \\
\hline 1614184_at & CF604220 & TC66293 & Q9SA77 & UDP-galactose 4-epimerase & Nucleotide metabolism & 21 & 2.13 \\
\hline 1618478_at & CF5I5277 & - & O64749 & UDP-galactose-4-epimerase & Nucleotide metabolism & 20 & 2.11 \\
\hline 1616383_at & CF609704 & TC59968 & Q8L9F5 & dTDP-glucose 4-6-dehydratase & Nucleotide metabolism & 21 & 2.06 \\
\hline |6158|4_at & CB920915 & TC56030 & Q7FAH2 & $\begin{array}{l}\text { Glyceraldehyde-3-phosphate } \\
\text { dehydrogenase }\end{array}$ & Phosphate Metabolism & 10 & 2.87 \\
\hline 16227I5_s_at & CA80928I & TC5 I78I & PI2858 & $\begin{array}{l}\text { Glyceraldehyde-3-phosphate } \\
\text { dehydrogenase }\end{array}$ & Phosphate Metabolism & 3 & 2.45 \\
\hline 1618277_at & CF568829 & TC56963 & Q8VWN9 & $\begin{array}{l}\text { Glyceraldehyde-3-phosphate } \\
\text { dehydrogenase }\end{array}$ & Phosphate Metabolism & 21 & 2.22 \\
\hline 1616083_at & CB009608 & TC51694 & Q9ZR63 & Hexose transporter $(\mathrm{VvHTI})$ & Transport & 2 & 12.37 \\
\hline 1610527_at & CA815926 & TC52979 & Q84QH3 & Sorbitol transporter & Transport & 2 & 5.49 \\
\hline 1615257_at & CB9727I3 & TC65400 & Q4U339 & Hexose transporter $\left(\mathrm{VvHT}_{\mathrm{v}}\right)$ & Transport & 15 & 4.7 \\
\hline 1619691_at & CF2II807 & TC62520 & Q4U339 & Hexose transporter ( $\mathrm{VvHT6})$ & Transport & 14 & 3.69 \\
\hline 1613408_at & CB347I78 & TC66667 & P93075 & Sucrose transporter (BvSTI) & Transport & II & 2.92 \\
\hline 160899I_at & CA816013 & TC60060 & Q8GTR0 & Sugar transporter & Transport & 10 & 2.86 \\
\hline 1610298_at & CB972367 & TC53493 & Q8LES0 & $\begin{array}{l}\text { Golgi nucleotide sugar transporter } \\
\text { (GONST) } 4\end{array}$ & Transport & 2 & 2.71 \\
\hline 1615697_at & AF021810 & TC51724 & Q4JLWI & Sucrose transporter (VvSuc27) & Transport & 3 & 2.44 \\
\hline 1611331_at & CF20I54I & TC69532 & Q69M22 & $\begin{array}{l}\text { Golgi nucleotide sugar transporter } \\
\text { (GONST) } 4\end{array}$ & Transport & 7 & 2.2 \\
\hline 161248|_at & CF2I 3270 & - & Q6ID34 & Glycerol 3-phosphate transporter & Transport & 4 & 2.03 \\
\hline
\end{tabular}


Furthermore, the transcript abundance of numerous transcripts involved in starch metabolism changed during berry development. One plastidial soluble starch synthase Unigene (1615571_at, TC53551) displayed increasing transcript abundance, while a second Unigene (1613601_at, TC67353) displayed decreasing transcript abundance during berry development (Figure 8D). A transcript for the plastidial $\alpha$-glucan, water dikinase ( $\mathrm{Gwd}$ ) gene (1617941_at, TC62494), which encodes an enzyme that is a regulator of starch mobilization and is essential for starch degradation [106], showed increased accumulation during berry development much like starch synthase I (1615571_at, TC53551). A second Gwd isogene (1617068_at, TC54621), showed peak transcript expression at E-L stage 35, but declined in fully ripe berries. Expression of plastidial $\alpha-1,4$ glucan phosphorylase (Starch phosphorylase L isozyme, 1622120_at, TC54533), a starch mobilization enzyme that phosphorylates amylopectin to catalyze the release of glucose-1phosphate, was nearly coordinate with the expression of this latter Gwd isogene. Finally, transcripts encoding the starch degrading enzymes, $\alpha$-amylase (1613188_at, TC70258) and $\beta$-amylase (1617124_at, TC67979), both showed increased abundance during berry development (Figure 8D). Grape berries are likely to contain intact and functional plastids at véraison and at later stages of ripeness as shown by in situ fixation of exocarp and mesocarp cells [107].

Figure 9 summarizes the major pathways of hexose sugars and polysaccharide flux and putative transport processes in the developing berry as defined by the combined transcriptomic and metabolite analyses performed in this study. Abridged gene expression patterns for key regulatory genes involved in both sucrose and starch metabolism are shown. One can easily visualize the coordinate transcript expression patterns for the entire pathway along berry development. It is not apparent from this analysis why fructose concentrations would be higher than glucose in berries. This indicates that the regulation of these hexoses by hexokinase genes, whose transcripts did not significantly change (data not shown), is more complex than what can be discerned from a simple examination of transcript profiles.

\section{Photosynthesis and carbon assimilation}

During berry development transcripts encoding proteins associated with photosynthesis-related functions are strongly expressed during the flowering stage and the socalled "herbaceous phase" or Phase I of berry development with expression declining during the later stages of berry maturation $[17,18]$. In our data, around 100 Unigenes with photosynthesis-related functions were identified with most displaying a steady or transient decline in the transcript abundance across berry development (Addi- tional file 5, Table 7). Similarly, transcripts encoding enzymes with roles in carbon assimilation also exhibited a declining pattern of expression. For instance, Unigenes encoding Calvin cycle enzymes such as glyceraldehyde-3phosphate dehydrogenase (1615814_at, TC56030; 1622715_s-at, TC51781), phosphoribulokinase (1614716_at, TC58640), transketolase (1618061_a_at, TC52548) as well as ribulose biphosphate carboxylase/ oxygenase small subunit (1612848_x_at, TC64044) were highly expressed and then declined during Phase III of berry development consistent with previous reports [18].

\section{Circadian cycles}

Circadian clocks are signaling networks that enhance an organism's growth, survival, and competitive advantage in rhythmic day/night environments [108]. The plant circadian clock modulates a wide range of physiological and biochemical events, such as stomatal and organ movements, photosynthesis and induction of flowering. A model of circadian rhythm based upon activities of several enzymes has been created involving transcription factors such as CIRCADIAN CLOCK-ASSOCIATED 1 (CCA1) or pseudo-response regulators such as PRR7 [108]. Transcripts for Unigene (1616834_at, TC54726) encoding CCA1 were repressed during the early stages of berry development, but increased in abundance at E-L stage 36 . In contrast, one Unigene (1608006_at, TC51808) related to the two-component response regulator APRR7 had a transient peak of expression in the early stages of berry development. This result is consistent with the position and function of these proteins in the circadian clock. Indeed, APRR7 represses CCA1 activity in Arabidopsis thaliana. In grape, these correlations in the transcript abundance indicate the operation of the circadian clock machinery throughout berry development. In addition, those genes are thought to enhance starch mobilization, consistent with previous observations made during Phase III of berry development [109].

\section{Pathogen and disease resistance related proteins}

Pathogen-related (PR) proteins are the most abundant class of proteins present in wine and can negatively affect the clarity and stability of wine [110]. During berry development, PR genes are expressed highly throughout various stages of berry growth. Around 30 Unigenes encoding different classes of PR genes were identified with a twofold ratio or greater expression change (Additional file 5, Table 8). Interestingly, four Unigenes encoding PR1 protein were highly expressed during early berry development, but then declined for the remainder of berry development. PR1 protein is regarded as one of the main down-stream responses of the salicylic acid signaling that plays an important role in Systemic Acquired Resistance. Salycylic acid is thought to accumulate just before véraison, which correlates well with the PR1 mRNA and protein 


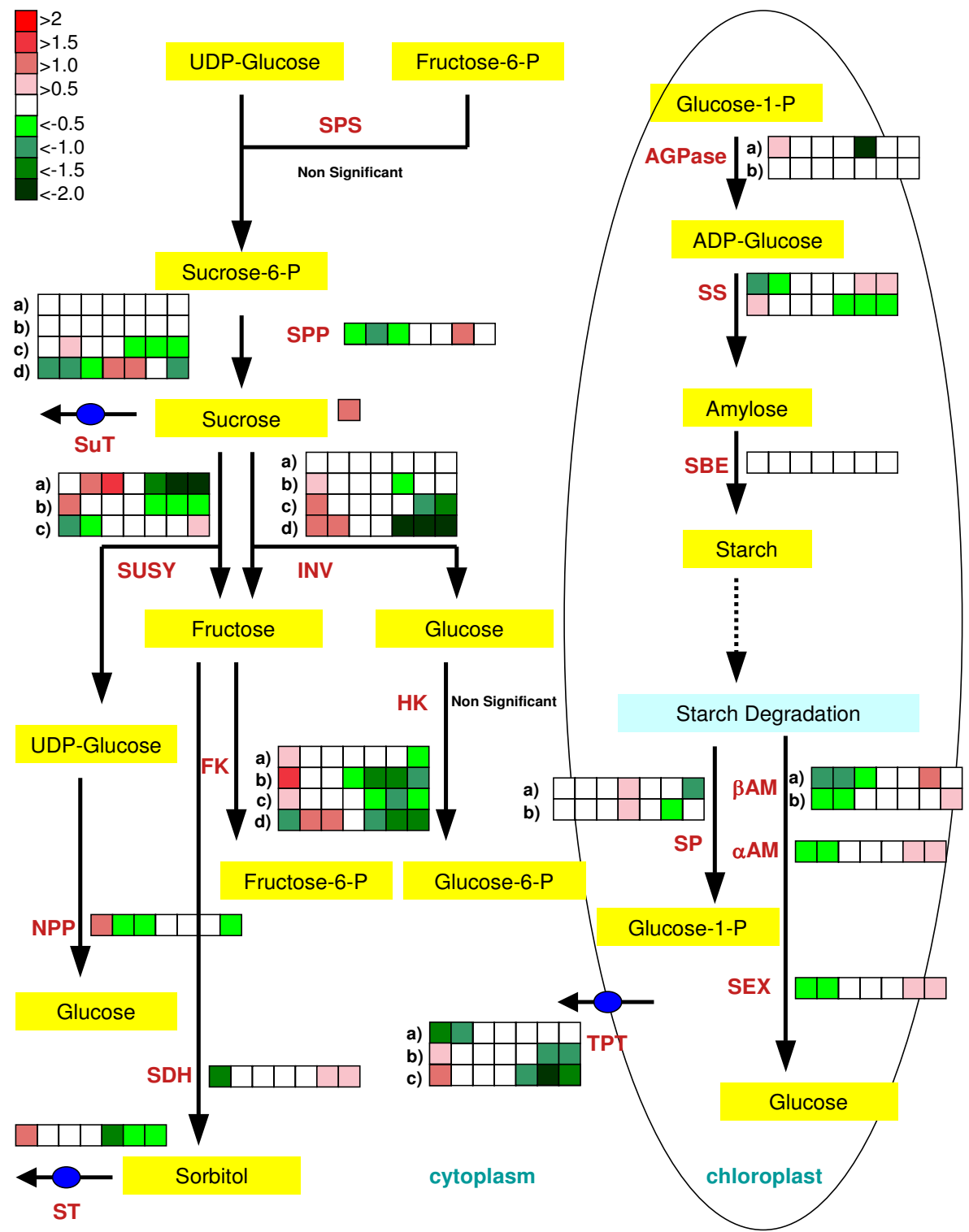

Figure 9

Transcriptomic mapping of transcripts related sucrose and starch metabolism along berry development. SPS: sucrose phosphate synthase-(I6I4674_at, TC60623), SPP: sucrose phosphate phosphorylase - (I608257_at, TC68I35) SUSY: sucrose synthase - a) (I616700_at, TC53526) b) (I619223_s_at, TC52910) c) (I609402_at, TC62599) INV: invertase - a) (I620628_at, TC67908) b) (I6II027_at, TC56057) c) (I6I2836_at, TC577I9) d) (I6II6I3_at, TC60693) HK: hexokinase-(16II4I9_at - TC533 I8) FK: fructokinase - a) (I628006_at, TC63769), b) (I622282_at, TC54393), c) (I62I053_at, TC63955), d) (I6I6255_at, TC57339) SuT: sucrose transporter - a) (I620256_at, AF02I808) b) (I62222I_at, AF02I809) c) (I615697_at, TC5I724) d) (16I5257_at, TC65400) NPP: nucleotide pyrophosphatase - (I620770_at, TC53085) SDH: sorbitol dehydrogenase - (1608527_at, TC58983) ST: sorbitol transporter - (1610527_at, TC52979) AGPase: ADP-glucose phosphatase - a) (1608393 at, TC64860) b) (1610928 at, TC64860) SBE: starch branching enzyme - (I62I790 at, TC6567I) SS: starch synthase - a) (I61557I_at, TC5355I) b) (I6I360I_at, TC67353) SP: starch phosphorylase - a) (1622120_at, TC54533) b) (1614707_at, TC53692) $\alpha$ AM: $\alpha$-amylase - (1613188_at, TC70258) $\beta$ AM: $\beta$-amylase - a) (1617I24_at, TC67979) b) (16I1808_at, CF205006) SEX: water dikinase - (161794I_at, TC62494) TPT: triose phosphate transporter - a) (160899I_at, TC60060) b) (1619379_at, TC5880I) c) (1622157_at, TC6I733). Each square from left to right corresponds to the expression of the probe sets from stage 31 through stage 38. Nonsignificant: Does not pass the ANOVA filter. 
Table 7: Transcripts (TFR pool) related to Energy metabolism within specific sub-sections

\begin{tabular}{|c|c|c|c|c|c|c|c|}
\hline Probeset ID & $\begin{array}{l}\text { GenBank } \\
\text { Annotation }\end{array}$ & VvGI5 & Uniprot ID & Gene Name Description & Function & Profile & Fold Change \\
\hline 1612882_at & CD720949 & TC6462I & A5BS4I & $\begin{array}{l}\text { ATP-dependent transmembrane } \\
\text { transporter }\end{array}$ & ATP binding & 2 & 5.55 \\
\hline 1612645_at & CB344I70 & TC55708 & P32980 & ATP synthase & ATP binding & 3 & 3.59 \\
\hline 1616533_at & CB339497 & TC62259 & P3 I853 & ATP synthase $B^{\prime}$ chain & ATP binding & 12 & 2.88 \\
\hline 1618182_at & CF604629 & TC63054 & PI9023 & ATP synthase beta chain & ATP binding & 21 & 2.23 \\
\hline 1607759_at & CD798264 & TC67523 & Q43433 & $\begin{array}{l}\text { Vacuolar ATP synthase subunit B } \\
\text { isoform } 2\end{array}$ & ATP binding & 11 & 2.16 \\
\hline 1620500_at & CB349662 & CB349662 & Q67IU5 & $\begin{array}{l}\text { Ribulose I,5-bisphosphate carboxylase } \\
\text { small subunit }\end{array}$ & Carbon dioxide fixation & 3 & 19.73 \\
\hline 1613936_x_at & CF568996 & CF568996 & O22077 & $\begin{array}{l}\text { Ribulose bisphosphate carboxylase } \\
\text { small chain }\end{array}$ & Carbon dioxide fixation & 3 & 2.36 \\
\hline 1616918_s_at & CB34554I & TC5639I & Q4028I & Rubisco activase & Carbon dioxide fixation & 3 & 2.22 \\
\hline 161968I_at & CD799678 & TC70996 & Q9C5C7 & Rubisco expression protein & Carbon dioxide fixation & 11 & 2.18 \\
\hline 1612848_x_at & CF202280 & CF202280 & PI0795 & Ribulose bisphosphate carboxylase & Carbon dioxide fixation & 3 & 2.13 \\
\hline |62055I_s_at & CB339855 & TC56836 & O98997 & Rubisco activase & Carbon dioxide fixation & 3 & 2.13 \\
\hline 161049|_at & CD010750 & TC57419 & Q8LFI7 & $\begin{array}{l}\text { Ribulose-I,5-bisphosphate } \\
\text { carboxylase/oxygenase }\end{array}$ & Carbon dioxide fixation & 12 & 2.13 \\
\hline 1616847_s_at & CA8I675I & TC68454 & O22077 & $\begin{array}{l}\text { Ribulose bisphosphate carboxylase } \\
\text { small chain }\end{array}$ & Carbon dioxide fixation & 3 & 2.12 \\
\hline 1616435_at & СВ974220 & TC68219 & P08927 & $\begin{array}{l}\text { RuBisCO subunit binding-protein beta } \\
\text { subunit }\end{array}$ & Carbon dioxide fixation & 3 & 2.1 \\
\hline 1622299_s_at & CKI36935 & CKI36935 & Q9LKH8 & $\begin{array}{l}\text { NADPH-protochlorophyllide } \\
\text { oxidoreductase }\end{array}$ & Chlorophyll biosynthesis & 3 & 5.3 \\
\hline 1619717_at & CF210684 & TC59048 & Q9SDTI & $\begin{array}{l}\text { NADPH:protochlorophyllide } \\
\text { oxidoreductase }\end{array}$ & Chlorophyll biosynthesis & 3 & 4.79 \\
\hline 1606624_at & CF606923 & TC64589 & Q43082 & Porphobilinogen deaminase & $\begin{array}{l}\text { Chlorophyll biosynthetic } \\
\text { process }\end{array}$ & 1 & 2.5 \\
\hline 1617935_at & CB974545 & TC68056 & Q7YJS8 & $\mathrm{NADH}$ dehydrogenase $49 \mathrm{kDa}$ subunit & Complex I & 16 & 5.55 \\
\hline 1611418 at & CB342953 & TC5616I & Q7YJ08 & $\mathrm{NAD}(\mathrm{P}) \mathrm{H}$-quinone oxidoreductase & Complex I & 14 & 5.09 \\
\hline 1609373_at & CD800734 & TC60468 & Q6KGYI & NADH dehydrogenase & Complex I & 14 & 3.05 \\
\hline 1614095_at & CF606244 & TC62268 & P0626I & $\mathrm{NAD}(\mathrm{P}) \mathrm{H}$-quinone oxidoreductase & Complex I & 21 & 2.83 \\
\hline |60942I_at & BQ795266 & TC628II & $0654 \mid 4$ & NADH dehydrogenase & Complex I & 11 & 2.75 \\
\hline 1617757_at & CA8I8465 & TC58524 & Q6YSN0 & NADH dehydrogenase & Complex I & 21 & 2.42 \\
\hline 1612005_s_at & СВ004075 & TC6467I & Q68S0I & NADH dehydrogenase & Complex I & 3 & 2.27 \\
\hline 1610869_at & CF5I5388 & TC56269 & Q8H2T7 & NADH dehydrogenase subunit & Complex I & 16 & 2.23 \\
\hline 1610347_s_at & CF202826 & CF202826 & Q0ZIW2 & $\mathrm{NAD}(\mathrm{P}) \mathrm{H}$-quinone oxidoreductase & Complex I & 10 & 2.1 \\
\hline 1609391_s_at & CF404650 & TC53103 & Q4I00I & Copper Binding Protein & Copper ion binding & 2 & 26.11 \\
\hline 1620588_at & CD80III57 & CD80III57 & Q8LED5 & Mavicyanin & Copper ion binding & 6 & 25.27 \\
\hline 1621220_at & CB919187 & TC59624 & Q9M510 & Dicyanin & Copper ion binding & 11 & 17.36 \\
\hline 1610220_at & CB97362I & TC68272 & 081500 & Copper Binding Protein & Copper ion binding & 3 & 14.36 \\
\hline 1617350_at & CB975555 & TC58747 & Q39131 & Copper Binding Protein & Copper ion binding & 3 & 10.21 \\
\hline 1611332_at & CF37I813 & TC59560 & Q653S5 & Blue copper binding protein (bcb) & Copper ion binding & I & 7.64 \\
\hline 1607270_at & CB923224 & TC60083 & Q9ZRV5 & Copper Binding Protein & Copper ion binding & 2 & 5.87 \\
\hline 1620744_at & CF403966 & TC65998 & PI7340 & Plastocyanin, chloroplast precursor & Copper ion binding & 3 & 4.41 \\
\hline 1617046_at & CF5I 2505 & TC54856 & O23230 & Trichohyalin & Copper ion binding & 3 & 2.23 \\
\hline 1609233_at & CF5I24I0 & TC54I70 & Q84RMI & Copper Binding Protein & Copper ion binding & 2 & 2.21 \\
\hline 1618207_at & CB347324 & TC52865 & Q9C540 & Cytochrome 56I & Electron carrier activity & 3 & 7.45 \\
\hline 1612624_at & CB974055 & TC58854 & P06449 & Apocytochrome f & Electron carrier activity & 21 & 5.32 \\
\hline 1611598 at & CB970208 & TC60594 & Q9ZSR3 & Cytochrome b-56I & Electron carrier activity & 2 & 4.54 \\
\hline 1606617_at & CF6080I0 & TC65350 & O23344 & Electron transport & electron carrier activity & 3 & 2.49 \\
\hline 1606704_s_at & CF200937 & CF200937 & P59702 & Cytochrome b559 alpha subunit & Electron carrier activity & 21 & 2.33 \\
\hline 1615927_s_at & СВ972I55 & TC55109 & Q6Q8B8 & Chloroplast ferredoxin I & Electron carrier activity & 3 & 2.23 \\
\hline 1607800_at & CB97252I & TC52149 & Q84WN3 & Cytochrome c oxidoreductase & Electron transport & 2 & 17.79 \\
\hline 1620504_at & CB342755 & TC52829 & Q84WN3 & cytochrome c oxidoreductase & Electron transport & 7 & 14.78 \\
\hline 1618535_at & CA818656 & CA8I8656 & Q6V5GI & $\mathrm{Cu} 2+$ plastocyanin & Electron transport & 13 & 6.25 \\
\hline 1615046_at & CF210436 & TC59116 & P4I346 & Ferredoxin--NADP reductase & Electron transport & 3 & 5.61 \\
\hline 1614266_at & BQ792322 & TC57I84 & Q49KU9 & $\begin{array}{l}\text { Cytochrome c heme attachment } \\
\text { protein }\end{array}$ & Electron transport & 16 & 4.17 \\
\hline 1613158_at & CB349843 & CB349843 & O47437 & Cytochrome c oxidase & Electron transport & 16 & 3.56 \\
\hline 1612766_s_at & CF569219 & CF569219 & Q5PY86 & NADH-cytochrome b5 reductase & Electron Transport & 3 & 3.32 \\
\hline
\end{tabular}


Table 7: Transcripts (TFR pool) related to Energy metabolism within specific sub-sections (Continued)

\begin{tabular}{|c|c|c|c|c|c|c|c|}
\hline 1619756_at & CB003378 & TC59085 & Q9LYC6 & Glutaredoxin & Electron transport & 14 & 2.88 \\
\hline |62099|_at & CB344999 & TC58191 & O24068 & Cytochrome oxidase subunit 3 & Electron transport & 16 & 2.26 \\
\hline 1606445_a_at & CF5I 2668 & TC62694 & P2629I & $\begin{array}{l}\text { Cytochrome B6-F complex iron-sulfur } \\
\text { subuni }\end{array}$ & Electron transport & 3 & 2.16 \\
\hline 1608372_at & CF20849I & TC51964 & Q6K7S7 & Cytochrome $\mathrm{c}$ biogenesis & Electron transport & 9 & 2.1 \\
\hline 1621402_a_at & CF213496 & TC5316I & P0005I & Cytochrome c & Electron transport & 11 & 2.01 \\
\hline 1607356_at & CB91 1288 & TC67262 & Q8LCF6 & Hypothetical Protein & ENERGY & 11 & 12.28 \\
\hline 1611972_s_at & CF519112 & TC53292 & $\mathrm{A} 4 \times 6 \mathrm{H} 5$ & Cytochrome b & ENERGY & 3 & 11.63 \\
\hline 1615762_at & CD798079 & TC66865 & 080763 & Hypothetical Protein & ENERGY & 10 & 4.82 \\
\hline 161624|_at & CD797326 & CD797326 & Q8VYC5 & Hypothetical Protein & ENERGY & 16 & 4.08 \\
\hline 1609285_at & CF4I4528 & TC57440 & Q9FFT2 & Hypothetical Protein & ENERGY & 2 & 3.44 \\
\hline 1611820_at & CB914713 & TC69253 & 080763 & Hypothetical Protein & ENERGY & 3 & 3.09 \\
\hline 1606562_at & CF404246 & TC594I5 & A3J369 & Nitrilase I & ENERGY & 11 & 3.04 \\
\hline 1621817_at & CB978007 & TC64650 & 080763 & Hypothetical Protein & ENERGY & 3 & 3 \\
\hline 1614875_at & CF518552 & TC69033 & A5AU55 & Hypothetical Protein & ENERGY & 11 & 2.73 \\
\hline 1622517_at & CB970523 & TC54809 & Q8W4Z5 & Hypothetical Protein & ENERGY & 3 & 2.39 \\
\hline 1621903_at & CF404558 & TC62294 & Q9FE29 & Hypothetical Protein & ENERGY & 13 & 2.23 \\
\hline 1612648_at & CD798203 & CD798203 & 080763 & Hypothetical Protein & ENERGY & 21 & 2.15 \\
\hline 1622345_at & CB970837 & TC55633 & Q7XTZ0 & Mandelonitrile lyase & Flavoprotein & 2 & 4.87 \\
\hline 1606948_at & CF404230 & CF404230 & Q0IJW7 & Mandelonitrile lyase & Flavoprotein & 2 & 3.68 \\
\hline 1622745_at & BQ796736 & TC58626 & Q8L5Q7 & Quinone oxidoreductase & FMN binding & 15 & 19.63 \\
\hline |6|548|_at & CB973026 & TC62178 & Q9ZSP7 & Cytochrome b5 DIF-F & Iron ion binding & 3 & 2.57 \\
\hline 1606727_at & BQ799998 & TC62672 & Q58IV4 & Phytochrome C & Light Signaling & 10 & 2.38 \\
\hline 1617604_at & CF609932 & TC59809 & Q94BM7 & $\begin{array}{l}\text { Phytochrome A supressor spal } \\
\text { protein }\end{array}$ & Light Signaling & 11 & 2.25 \\
\hline 1611135_at & CB983077 & TC5I9II & Q9SG92 & Alpha-hydroxynitrile lyase & Lyase activity & 11 & 2.92 \\
\hline 1622108_at & CF405863 & TC56579 & Q9SU40 & Monocopper oxidase & $\begin{array}{l}\text { Multicopper oxidase } \\
\text { family }\end{array}$ & 3 & 23.07 \\
\hline 1621115_at & CF609165 & TC64I36 & Q9SU40 & Monocopper oxidase & $\begin{array}{l}\text { Multicopper oxidase } \\
\text { family }\end{array}$ & 1 & 20.1 \\
\hline 1617992_a_at & CF2I367I & TC60094 & P5II32 & $\begin{array}{l}\text { Ubiquinol--cytochrome-c reductase- } \\
\text { like protein }\end{array}$ & $\begin{array}{l}\text { Oxidative } \\
\text { Phosphorespiration }\end{array}$ & 11 & 2.18 \\
\hline 1611597_at & CB918250 & CB918250 & Q8LDU4 & Red chlorophyll catabolite reductase & Oxidoreductase activity & 12 & 2.03 \\
\hline 1613786_at & CD7I4955 & TC57282 & Q6QY10 & P700 chlorophyll a apoprotein AI & Photosystem I & 21 & 8.65 \\
\hline 1611364_at & CF2III293 & TC52528 & Q9XQB4 & Reaction center subunit III & Photosystem I & 3 & 7.67 \\
\hline 1611464_at & CF215949 & TC59235 & Q9XF85 & Lhca5 protein & Photosystem I & 3 & 7.21 \\
\hline 1621532_at & CB97372I & TC64270 & Q84QE6 & Reaction center subunit $X$ psaK & Photosystem I & 3 & 7.15 \\
\hline 1619903_at & CD720479 & TC65556 & Q405I2 & $\begin{array}{l}\text { Light-harvesting chlorophyll a/b-binding } \\
\text { protein }\end{array}$ & Photosystem I & 3 & 6.96 \\
\hline 1619629_at & CB340944 & TC66352 & Q5DNZ6 & Chlorophyll a-b binding protein & Photosystem I & 3 & 6.59 \\
\hline 1622534_at & BQ799942 & TC53444 & Q84U30 & Photosystem I-N subunit & Photosystem I & 3 & 6.45 \\
\hline 1611733_s_at & BQ797982 & TC52546 & Q70PN9 & $\begin{array}{l}\text { Reaction centre PSI-D subunit } \\
\text { precursor }\end{array}$ & Photosystem I & 3 & 6.44 \\
\hline 1616560_at & CA8I7733 & TC62550 & Q84WTI & $\begin{array}{l}\text { Light-harvesting chlorophyll a/b binding } \\
\text { protein }\end{array}$ & Photosystem I & 3 & 5.9 \\
\hline 1611515_s_at & CB343423 & TC57304 & O65I0I & Reaction center subunit $\mathrm{VI}$ & Photosystem I & 3 & 5.33 \\
\hline 1618370_at & CF510718 & TC57721 & Q9SU14 & Reaction center subunit XI & Photosystem I & 3 & 5.31 \\
\hline 161777I_at & CF4I4I58 & TC58342 & Q8RVJ8 & Reaction centre subunit IV & Photosystem I & 3 & 4.87 \\
\hline 1618127_at & CB968637 & TC62932 & Q9SY97 & Chlorophyll a/b-binding protein & Photosystem I & 3 & 4.74 \\
\hline 1614409_at & CA8I7387 & TC55189 & PI3869 & Chlorophyll a-b binding protein & Photosystem I & 3 & 4.65 \\
\hline 1614593_at & CF5III805 & TC52379 & Q00321 & CP29 polypeptide & Photosystem I & 3 & 4.43 \\
\hline 1611924_at & CA8I7406 & TC63702 & $\mathrm{Q} 646 \mathrm{H} 3$ & Reaction center $\mathrm{V}$ & Photosystem I & 3 & 4.06 \\
\hline 1611161 at & CF210442 & TC54044 & Q9ZU86 & Expressed protein & Photosystem I & 3 & 3.09 \\
\hline 1622302_s_at & CF207602 & TC54765 & Q40459 & Oxygen-evolving enhancer protein I & Photosystem I & 3 & 2.8 \\
\hline 1610245_at & CF209798 & TC53968 & Q41424 & Chlorophyll a/b binding protein & Photosystem II & 3 & 15.82 \\
\hline 1615822_at & CF20832I & TC52049 & Q9XQBI & $\begin{array}{l}\text { LHCII type III chlorophyll a/b binding } \\
\text { protein }\end{array}$ & Photosystem II & 3 & 10.43 \\
\hline |6083|I_at & CF2025I9 & CF2025I9 & Q7MIK9 & Chlorophyll a/b-binding protein & Photosystem II & 3 & 10.24 \\
\hline 1616940_s_at & CB348709 & TC52113 & Q7MIK9 & Chlorophyll a/b-binding protein & Photosystem II & 3 & 9.95 \\
\hline 1618116_s_at & BQ798823 & TC55659 & Q3229I & $\begin{array}{l}\text { Chlorophyll A/B binding protein } \\
\text { precursor }\end{array}$ & Photosystem II & 3 & 7.28 \\
\hline 1611860_at & CF209952 & TC5752I & Q9XQB6 & Chlorophyll a/b-binding protein CP24 & Photosystem II & 3 & 6.6 \\
\hline 1612085_at & CF4I3799 & TC54542 & Q41387 & Reaction center $\mathrm{W}$ protein & Photosystem II & 3 & 5.79 \\
\hline
\end{tabular}


Table 7: Transcripts (TFR pool) related to Energy metabolism within specific sub-sections (Continued)

\begin{tabular}{|c|c|c|c|c|c|c|c|}
\hline 1621038_at & CF372077 & TC57214 & O64448 & $\begin{array}{l}\text { Light harvesting chlorophyll a/b-binding } \\
\text { protein precursor }\end{array}$ & Photosystem II & 3 & 5.77 \\
\hline 1618679_s_at & CB343106 & TC52042 & Q9BBTI & $44 \mathrm{kDa}$ reaction center protein & Photosystem II & 16 & 5.41 \\
\hline 1610203_at & CD009386 & TC56267 & Q7YJY8 & Photosystem Q(B) protein & Photosystem II & 21 & 5.11 \\
\hline 161399|_at & CF510955 & TC53743 & P80470 & Core complex proteins psbY & Photosystem II & 3 & 4.77 \\
\hline 1607516_at & CB972913 & TC53930 & Q9LRC4 & $\begin{array}{l}\text { Oxygen evolving enhancer protein I } \\
\text { precursor }\end{array}$ & Photosystem II & 3 & 4.66 \\
\hline 1613428_at & CF207I58 & TC52084 & Q5PYQ5 & $\begin{array}{l}\text { Chloroplast oxygen-evolving enhancer } \\
\text { protein }\end{array}$ & Photosystem II & 3 & 4.47 \\
\hline 160796I_at & CF4I57I6 & TC57429 & P31336 & $5 \mathrm{kDa}$ protein & Photosystem II & 3 & 4.16 \\
\hline 1614598_at & CF373065 & TC61762 & Q9XQB2 & Chlorophyll a/b binding protein CP29 & Photosystem II & 3 & 4.06 \\
\hline 161369|_s_at & CF5II746 & TC54828 & P275। 8 & Chlorophyll a-b binding protein I5I & Photosystem II & 3 & 3.84 \\
\hline 1613773_s_at & BQ799|45 & TC63656 & Q4I387 & Reaction center $W$ protein & Photosystem II & 3 & 3.34 \\
\hline 1618031_s_at & CF40445I & TC53833 & Q8GV53 & $10 \mathrm{kDa}$ protein & Photosystem II & 3 & 3.28 \\
\hline |62135|_s_at & CB340283 & TC53732 & Q4096I & $\begin{array}{l}\text { Light-harvesting chlorophyll a/b-binding } \\
\text { protein precursor }\end{array}$ & Photosystem II & 3 & 3.2 \\
\hline 1613494_s_at & CA8I3944 & TC55522 & Q9SLQ8 & Oxygen-evolving enhancer protein 2 & Photosystem II & 3 & 3.05 \\
\hline 1617605_at & CF5I3977 & TC55526 & Q8HS34 & CP47 protein & Photosystem II & 18 & 2.99 \\
\hline 1618274_at & CB97247I & TC55538 & Q4FFQ9 & Phosphoprotein & Photosystem II & 21 & 2.92 \\
\hline 1610144_at & CB342508 & TC5359I & Q9MTN0 & $\begin{array}{l}\text { Uncharacterized } 6.9 \mathrm{kDa} \text { protein in } \\
\text { psbD-trnT intergenic region }\end{array}$ & Photosystem II & 15 & 2.88 \\
\hline 161|582_s_at & CB970190 & TC70959 & Q02060 & $22 \mathrm{kDa}$ protein & Photosystem II & 3 & 2.69 \\
\hline 1607926_at & CF202256 & CF202256 & Q9AR57 & Putative membrane protein & Photosystem II & 2 & 2.56 \\
\hline 1621978_at & CB837910 & TC56626 & Q9M3M7 & Uncharacterized protein & Photosystem II & 16 & 2.31 \\
\hline 1607803_at & CB975690 & TC52112 & Q06364 & $\begin{array}{l}26 \mathrm{~S} \text { proteasome non-ATPase } \\
\text { regulatory subunit } 3\end{array}$ & Photosystem II & 21 & 2.31 \\
\hline 1619523_at & CB969438 & TC67627 & Q952RI & Succinate dehydrogenase & $\begin{array}{l}\text { Succinate } \\
\text { dehydrogenase activity }\end{array}$ & 15 & 2.24 \\
\hline
\end{tabular}


Table 8: Transcripts (TFR pool) related to Pathogenesis-Related proteins within specific sub-sections

\begin{tabular}{|c|c|c|c|c|c|c|c|}
\hline Probeset ID & $\begin{array}{l}\text { GenBank } \\
\text { Annotation }\end{array}$ & VvGI5 & Uniprot ID & Gene Name Description & Category & Profile & Fold Change \\
\hline 161347|_at & CF2I5857 & TC59306 & Q9SW05 & Pathogenesis-related protein & PRI & 3 & 7.83 \\
\hline 1611058_at & CA8I4I53 & TC67060 & Q7XAJ6 & Pathogenesis related protein I & PRI & 3 & 5.76 \\
\hline 1613816_x_at & CF074673 & TC56938 & Q7XAJ6 & Pathogenesis related protein I & PRI & 21 & 4.74 \\
\hline 1618533_at & CB970020 & TC55782 & Q40374 & Pathogenesis related protein I & PRI & 2 & 2.31 \\
\hline 1615595_at & AF239617 & AF239617 & Q9M563 & $\beta$-I,3-glucanase & PR2 & 11 & 10.99 \\
\hline 1620496_at & CF214365 & TC66187 & Q8VYI2 & $\beta$-I,3-glucanase & PR2 & 15 & 2.3 \\
\hline 1608203_at & CF5III34 & TC64974 & Q94EN5 & $\beta$-I,3-glucanase & PR2 & 2 & 2.17 \\
\hline 1616183_at & CF405742 & TC62849 & Q94G86 & $\beta$-I,3-glucanase & PR2 & 14 & 2.17 \\
\hline 1610324_a_at & CB34604I & TC6705I & Q8L868 & $\beta$-I,3-glucanase & PR2 & 12 & 2.05 \\
\hline 1621319_s_at & CB98II 22 & TC70080 & Q7XAU6 & Chitinase IV & PR3 & 10 & 299.34 \\
\hline 161346I_s_at & AF532966 & AF532966 & Q7XAU6 & Chitinase IV & PR3 & 10 & 162.58 \\
\hline 1607557_at & CF202548 & CF202548 & Q7XAU6 & Chitinase IV & PR3 & 10 & 149.38 \\
\hline |61455|_at & CB3437I5 & TC5I734 & Q6JX04 & Chitinase & PR3 & 2 & 49.79 \\
\hline 1616064_at & CF205270 & CF205270 & O2453I & Chitinase IV & PR3 & II & 20.19 \\
\hline 1621583_at & CF404733 & TC62834 & Q6JX04 & Chitinase & PR3 & 1 & 4.93 \\
\hline 1620111_at & CF568854 & CF568854 & Q6JX04 & Chitinase & PR3 & 2 & 3.36 \\
\hline 1606625_at & CF603972 & TC64563 & Q7XB39 & Chitinase IV & PR3 & 19 & 3.21 \\
\hline 16205|8_at & CF20I34I & CF20I34I & 081228 & Pathogenesis related protein 4 & PR4 & 15 & 43.11 \\
\hline 1618835_s_at & BQ797I63 & TC58333 & 081228 & Pathogenesis related protein 5 & PR4 & 15 & 25.9 \\
\hline 1612160_at & CF4I5249 & TC646II & P50699 & Thaumatin & PR5 & 3 & 34.49 \\
\hline 161887|_at & CF5I055I & TC55284 & Q82L96 & Thaumatin & PR5 & 3 & 15.65 \\
\hline 1616617_at & AFI 95654 & AFI95654 & Q9SNY0 & Thaumatin & PR5 & 11 & 11.86 \\
\hline 1607225_at & CB914105 & TC65548 & O65638 & Thaumatin & PR5 & 11 & 8.51 \\
\hline 1614746_at & CF214284 & TC53053 & Q7XST4 & Thaumatin & PR5 & 2 & 5.27 \\
\hline 1607708_at & CF4I384I & TC63I77 & Q9LZL8 & Thaumatin & PR5 & 2 & 4.22 \\
\hline 1606517_at & CB347I9I & TC62530 & Q8LBL4 & Thaumatin-like protein & PR5 & 14 & 3.51 \\
\hline 1622374_at & CB920589 & TC56535 & Q41350 & Thaumatin & PR5 & 2 & 3.34 \\
\hline 1613999_x_at & CF202364 & CF202364 & Q84S31 & Chitinase III & PR8 & 2 & 4.57 \\
\hline
\end{tabular}

expression [111]. The two main PR proteins that have a significant role in the defense against invading fungal pathogens are $\beta$-1,3-glucanase (PR2) and chitinase (PR3) [112]. Five Unigenes encoding $\beta$-1,3-glucanase were transiently expressed at different periods of berry development. Unigenes encoding various chitinases were also identified that displayed similar mRNA expression patterns. Some chitinase genes exhibit strong homologies with a chitinase previously observed in grape berry [111]. Another PR protein, which may play a role in grape berry defense, is thaumatin protein (PR5) [113]. Eight Unigenes encoding PR5 proteins were identified and their respective expression patterns span all stages of berry development. Taken together, the expression patterns revealed that these defense-related gene products and enzymes are expressed across all stages of berry development. Such a Systemic Acquired Resistance strategy probably minimizes pathogen invasion as previously suggested [114].

\section{Quantitative real-time RT-PCR}

To validate expression profiles obtained using the Affymetrix GeneChip ${ }^{\circledast}$ Vitis genome array, quantitative real-time RT-PCR was performed on 11 genes using gene-specific primers [Additional file 5, Table 3]. Transcript abundance patterns were calculated along the entire course of berry development. Linear regression ([microarray value] = a[RT-PCR value]+b) analysis showed an overall correlation coefficient of 0.94 indicating a good correlation between transcript abundance assessed by real-time RTPCR and the expression profiles obtained with the GeneChip ${ }^{\circledast}$ genome arrays (Figure 10).

\section{Conclusion}

Our large-scale transcriptomic analysis demonstrated that nearly a third $(28 \%)$ of genes expressed in berries exhibited at least two-fold or greater change in steady-state transcript abundance over the course of seven stages of grape berry development. Approximately two-thirds (64\%) of these Unigenes could be assigned a functional annotation with the remaining one-third having obscure or unknown functions. Twenty distinct patterns of expression were resolved in order to illustrate the complex transcriptional regulatory hierarchies that exist to orchestrate the dynamic metabolic, transport, and control processes occurring in developing berries. We provided evidence that phytohormone biosynthesis and responses, particularly for ABA, ethylene, brassinosteroids, and auxins, as well as calcium homeostasis, transport, and signaling 


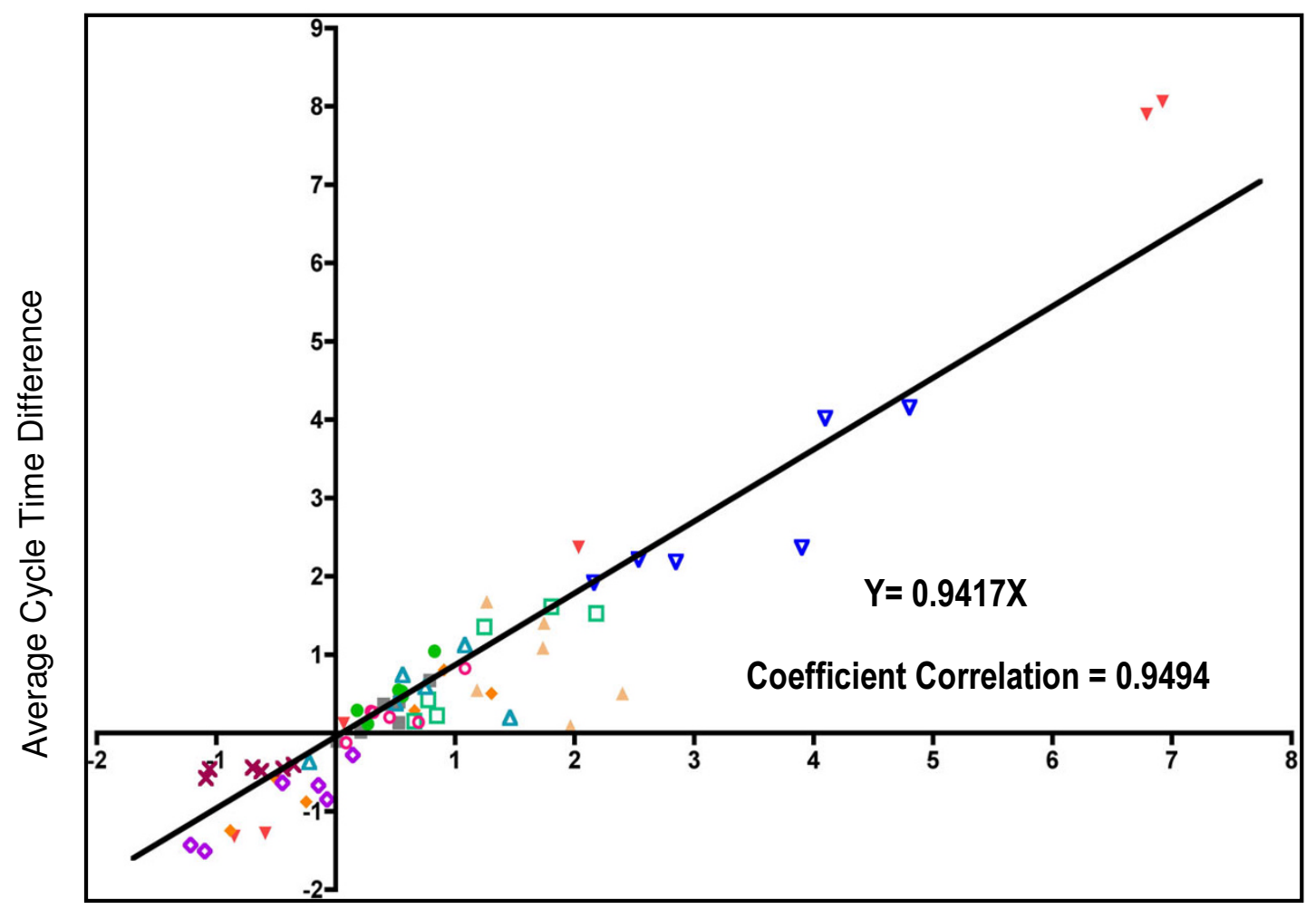

Average Signal $\log _{2}$ Ratio

Figure 10

Quantitative real-time RT-PCR of eleven transcripts. Comparison between the gene expression ratios reported by the Affymetrix GeneChip ${ }^{\circledR}$ genome array and by real-time RT-PCR. Data were from II probe sets across seven developmental stages. The difference in the number of PCR cycles required to produce the same amount of product is plotted against the log 2 expression ratio averaged over the first time point. The linear regression line was constrained to pass through the origin. Grey solid square (1615402_at, TC56083)-ferulate-5-hydroxylase, Apricot solid triangle (1606794_at, TC6389I)-osmotin precursor, red solid triangle (1616700_at, TC53526)-sucrose synthase, orange solid diamond (1607760_at, TC5 I695) flavonoid-3'5'hydroxylase, light green solid round (161 1650_at, TC57228)-WRKY7, dark green open square (I6I6880_at, TC54034)-cinnamoyl alcohol dehydrogenase, dark blue open triangle (1613896_at, TC62 182)-nitrate/chloride transporter), blue open triangle (I6I5722_s_at, TC5 I 776)-aquaporin PIPI.I, lavender open diamond ( 6 I I 342_at, TC55943)-serine/threonine kinase, pink open circle (16I2I32_s_at, TC683 I I)-protein phosphatase 2C, brown cross (I6I493I_at, TC6I058)-MYB transcription factor.

processes play critical roles in this developmental process. We also demonstrate that the expression and regulation of genes involved in cell wall biosynthesis and expansion, as well as genes involved in the biosynthesis, transport, and regulation of the phenylpropanoid and flavonoid pathways undergo dynamic changes throughout the course of berry development. Our analysis has revealed candidate genes that may participate in the production of different classes of aroma producing compounds. We have also demonstrated coordinate regulation of transcripts and the accumulation of key metabolites including tartrate, malate, and proline during berry development. A close examination of the behavior of gene expression patterns of genes involved in sugar and starch metabolism indicate that plastidial starch reserves are mobilized to fuel the production of hexose sugars during the ripening and maturation phase (Phase III) of berry development. Finally, our findings provide the first functional genomic information for hundreds of genes with obscure functions that can be exploited for hypothesis testing by traditional functional assays to improve our understanding of the complex developmental processes present in grape berries and to ultimately utilize this information to improve quality traits of wine grapes. 


\section{Methods \\ Plant Materials}

Six twenty-year-old Cabernet Sauvignon (Vitis vinifera L.) vines grown on St. George rootstock were used during 2004 for this study. The vines were located at the Shenandoah Vineyard in Plymouth, CA, on a hillside row located in the middle of the vineyard. All plants were equipped with a drip irrigation system and watered daily to keep their water status high. Mid-day stem water potentials were measured weekly with a pressure chamber on two mature leaves per plant for a total of 6 vines [115]. For each measurement, a single leaf per plant was tightly zipped in a plastic bag to eliminate transpiration and covered with aluminum foil to deflect light and heat. After two hours of equilibration time, the petiole was cleanly cut and carefully threaded through a rubber gasket in the lid of a pressure chamber (3005 Plant Water Status Console, Soilmoisture Equipment Corp., Santa Barbara, CA, USA). The foil was removed before sealing the bagged leaf in the chamber. The balancing pressure required to visibly push stem xylem sap to the cut surface was recorded.

Two grape clusters were sampled weekly from each plant ( $n=6)$, one from the south (sunny) and one from the north (shady) side of the plant. The clusters were pooled together for each plant in order to avoid light and temperature effects. Berry development was characterized by monitoring berry diameter, total soluble solids and titratable acidity. The berry diameter was measured with a micrometer for fifteen randomly selected berries per each of two clusters and an average berry size was computed per vine $(n=6)$. Total soluble solids ( ${ }^{\circ}$ Brix) were assayed (two technical replicates) with a refractometer (BRIX30, USA) from juice crushed from harvested berries from two clusters per vine $(n=6)$ to estimate total sugar content. Titratable acidity $(\mathrm{g} / \mathrm{L})$ of the grape juice was measured by titration to an endpoint of $\mathrm{pH} 8.4$ with a strong base. The same number of repetitions as in ${ }^{\circ}$ Brix measurements was used.

\section{RNA extraction and microarray hybridization}

Total RNA was extracted from berries finely ground in liquid nitrogen using Qiagen RNeasy Plant MidiKit columns (Qiagen Inc., CA) as previously described [116]. The total RNA was further purified using a Qiagen RNeasy Plant Mini Kit (Qiagen, Valencia, CA) according to the manufacturers' instructions. RNA integrity was confirmed by electrophoresis on $1.5 \%$ agarose gels containing formaldehyde and quality was confirmed by analysis on an Agilent 2100 Bioanalyzer using RNA LabChip ${ }^{\circledR}$ assays according to the manufacturer's instructions. mRNAs were converted to $\mathrm{cDNAs}$ using oligo dT primer containing a T7 RNA polymerase promoter sequence and reverse transcriptase. Biotinylated complementary RNAs (cRNAs) were synthesized in vitro using T7 RNA polymerase in the presence of biotin-labeled UTP/CTP, purified, fragmented and hybridized in the GeneChip ${ }^{\circledR}$ Vitis vinifera Genome Array cartridge (Affymetrix ${ }^{\circledast}$, Santa Clara, CA). The hybridized arrays were washed and stained with streptavidin phycoerythrin and biotinylated anti-streptavidin antibody using an Affymetrix Fluidics Station 400. Microarrays were scanned using a Hewlett-Packard GeneArray ${ }^{\circledR}$ Scanner and image data was collected and processed on a GeneChip ${ }^{\circledR}$ workstation using Affymetrix ${ }^{\circledR}$ GCOS software.

\section{Microarray data processing}

Three biological replicates per experiment were processed to evaluate intra-specific variability. Expression data were processed by RMA (Robust Multi-Array Average) [117] using the R package affy [118]. Specifically, the RMA model of probe-specific background correction was first applied to the PM (perfect match) probes. These corrected probe values were normalized via quantile normalization and a median polish method was applied to compute one expression measure from all probe values. Data quality was verified by digestion curves describing trends in RNA degradation between the 5 ' end and the 3 ' end in each probe set. Differentially expressed genes throughout berry development were determined by ANOVA on the RMA expression values [118]. A multiple testing correction [22] was applied to the $p$-values of the F-statistics to adjust the false discovery rate. Genes with adjusted $p$-values $<0.05$ were extracted for further analysis. Genes having a twofold ratio (TFR) or greater between at least two time points along berry development were selected for further analyses. The RMA expression data (experiment Vv5) have been deposited in PLEXdb [119].

\section{Microarray data analysis}

Clustering of co-regulated genes was performed using the MultiExperimentViewer software part of the TM4 software package (MEV3.1) developed by TIGR [120]. TFR Unigenes were clustered via the Pavlidis Template Matching (PTM) algorithm [24]. The twenty template profiles were selected (by us) as representatives of biological processes occurring during berry development (Additional File 4). The Pearson correlation coefficient between each Unigene and each template profile was used to determine cluster membership: correlation measures greater than 0.75 corresponded to a good match. If genes were well correlated with more than one template profile, the gene was assigned to the cluster with which it had greatest correlation. The $p$-values associated to the hypothesis test of each correlation coefficient (null hypothesis is that the correlation is zero) were calculated and a multiple testing correction (Benjamini and Hochberg) was applied. Only genes with adjusted $p$-values $\leq 0.05$ and correlations greater than 0.75 were placed into clusters 


\section{Unigene Annotation and Functional Analysis}

Unigene annotation was updated by nucleotide sequence query of the probe consensus sequence against the UniProt/TrEMBL, NCBI-nr and TAIR protein databases using BLASTX (e-value < 1e-05). Functional categories were assigned automatically by amino acid homology to Arabidopsis thaliana proteins categorized according to the Munich Information Center for Protein Sequences (MIPS) Funcat 2 classification scheme [25]. Bibliographic searches were performed to assign functions to Unigenes exhibiting no homology with Arabidopsis thaliana proteins. Some annotation presented here will be subject to error due to the relatively correlative nature of these associations. It is expected that the annotated data presented here will be used for future hypothesis-driven research that can establish stronger functional analyses and annotations.

Attribution of the 20 clusters to the key developmental phases (I, II or III) (See Figure 6) was decided according to two criteria. The first one was to fit these phases with the time points used in this study. Stage 31 (Modified E-L System) was the only one belonging to the herbaceous phase (Phase I). The lag phase (Phase II) corresponded to stages 32 to 34 . The maturation phase (Phase III) included stages 35 to 38 . The second criterion was based on the time point at which the maximum average gene expression value was observed across the genes within each cluster. For instance, cluster 1 was included in the Phase I group, because the maximum average expression level was observed at stage 31 . The same assignments were made in the other phases (II and III) (See Additional File 5: Table 1 ). To test for significant differences in the representation of Unigenes within each functional category per developmental phase (Phases I, II and III; see Figure 5), a Pearson's chi-squared test was used [121]. Three comparisons (Phase I against II; I against III and II against III) were performed and results are listed in Additional File 5: Table 2. Differences in frequency for each category between two stages were considered significant for a $p$-value $<0.05$.

\section{Real Time PCR experiments}

RNA was extracted and its integrity verified by standard procedures. cDNA was synthesized using an iScript cDNA Synthesis Kit (Bio-Rad Laboratories, Hercules, CA) according to the manufacturer's instructions with a uniform $1 \mu \mathrm{g}$ RNA per reaction volume reverse-transcribed. Primers for genes (Additional File 5: Table 3) assayed by real-time PCR were selected using Primer3 software [122]. Quantitative real-time PCR reactions were prepared using an iTaq SYBR Green Supermix with ROX (Bio-Rad) and performed using the ABI PRISM ${ }^{\circledast} 7000$ Sequence Detection System (Applied Biosystems, Foster City, CA). Expression was determined for triplicate biological replicates by use of serial dilution cDNA standard curves per gene. In order to assess the performance of the array in a biological context, we examined the transcript abundance of some candidate genes from Cabernet Sauvignon exhibiting changing expression patterns across the 7 time points of berry development. Real-time RT-PCR was performed with the ABI PRISM ${ }^{\circledR} 7000$ Sequence Detection System (Applied Biosystems, Forster City, CA) under annealing conditions of $50^{\circ} \mathrm{C}$ for 1 minute and analyzed with $\mathrm{ABI}$ PRISM $^{\circledR} 7000$ SDS software. Analysis of relative gene expression was performed using the $2^{-\Delta \Delta \mathrm{C}_{T}}$ method [123]. The data were analyzed using the equation $\Delta \Delta \mathrm{C}_{T}=$ $\left(\mathrm{C}_{\mathrm{T}, \text { Target }}-\mathrm{C}_{\mathrm{T}, \mathrm{HG}}\right)$ Time $X$ - $\left(\mathrm{C}_{\mathrm{T}, \text { Target }}-\mathrm{C}_{\mathrm{T}, \mathrm{HG}}\right)$ Time 0 where Time $X$ is the value at any time point and Time 0 represents the $1 \mathrm{X}$ expression of the target gene normalized to ankyrin. Data were calculated from the calibration curve and normalized using the expression curve of an ankyrin gene (1612584_s_at; TC53110), whose mRNA presented an extremely low coefficient of variation (0.056, M Value $=0.1297)$ through microarray analysis [124].

\section{Metabolite extraction and derivatization}

Polar metabolites were extracted and derivatized with a water/chloroform protocol according to previously established procedures [125]. Freeze-dried berry tissue (6 mg) was placed in a standard screw-cap-threaded, glass vial. The tube was then returned to the $-80^{\circ} \mathrm{C}$ freezer until use. Frozen tubes were wrapped in parafilm and freeze-dried overnight. All tissue samples were kept frozen throughout the lyophilization procedure. Upon lyophilization, tubes were capped and returned to the freezer until extraction. The vials were allowed to cool back to room temperature before being handled. The extraction vials were not washed with a methanol/hexane rinse, but all caps and septa were. The vial was incubated in HPLC grade chloroform for 1 hour at $50^{\circ} \mathrm{C}$ in an oven. A volume of Millipore water was added $(\mathrm{m} / \mathrm{V})$ containing $25 \mathrm{mg} / \mathrm{L}$ of ribitol as an internal standard and the sample was re-incubated for an additional hour at $50^{\circ} \mathrm{C}$. Finally, vials were allowed to cool to room temperature and then spun down at 2,900 $\times$ $\mathrm{g}$ for 30 minutes. One $\mathrm{mL}$ of the polar phase was dried down in a vacuum concentrator. Polar samples were derivatized by adding $120 \mu \mathrm{L}$ of $15 \mathrm{mg} \mathrm{mL}^{-1}$ of methoxyamine $\mathrm{HCl}$ in pyridine, incubated at $50^{\circ} \mathrm{C}$ for 30 minutes and sonicated until all crystals disappeared. After that, $120 \mu \mathrm{L}$ of MSTFA $+1 \%$ TMCS were added, incubated at $50^{\circ} \mathrm{C}$ for 30 minutes and immediately submitted for analysis with a Thermo Finnigan Polaris Q230 GC-MS (Thermo Electron Corporation, San Jose, CA, USA). The inlet and transfer lines were held at $240^{\circ} \mathrm{C}$ and $320^{\circ} \mathrm{C}$, respectively. Separation was achieved with a temperature program of $80^{\circ} \mathrm{C}$ for $3 \mathrm{~min}$, then ramped at $5^{\circ} \mathrm{C} \mathrm{min}^{-1}$ to $315^{\circ} \mathrm{C}$ and 
held for $17 \mathrm{~min}$, using a $60 \mathrm{~m}$ DB-5MS column (J\&W Scientific, $0.25 \mathrm{~mm}$ ID, $0.25 \mu \mathrm{m}$ film thickness) and a constant flow of $1.0 \mathrm{ml} \mathrm{min}{ }^{-1}$. Derivatized samples $(120 \mu \mathrm{L})$ were transferred to a $200 \mu \mathrm{L}$ silanized vial insert and run at an injection split of 200:1 to bring the large peaks to a concentration within the range of the detector. Identity of all organic acids, sugars and amino acids were verified by comparison with standards purchased from SigmaAldrich (St. Louis, MO, USA).

\section{Metabolite data processing}

Metabolites were identified from the chromatograms using two different software packages: AMDIS (2.64, United States Department of Defense, USA) and Xcalibur (1.3; Thermo Electron Corporation). The software matched the mass spectrum in each peak against three different metabolite libraries: NIST ver. 2.0 library [126], T_MSRI_ID library of the Golm Metabolome Database [127] and our own custom-created UNR library (V1) made from more than 50 standards bought from SigmaAldrich. Quantification of the area of the chromatogram peaks was determined using Xcalibur and normalized as a ratio of the area of the peak of the ribitol internal standard.

\section{Starch determination}

Starch assays were performed according to Dubois et al. [128]; $100 \mathrm{mg}$ of berry powder from E-L stages (35 to 38) were finely ground and incubated in $5 \mathrm{~mL}$ of methanol $(80 / 20 ; \mathrm{v} / \mathrm{v})$ at $80^{\circ} \mathrm{C}$ for $40 \mathrm{~min}$. This step eliminates soluble sugars. The methanol extract was removed and the pellet was washed twice with distilled water. The remaining pellet was incubated overnight in $1.2 \mathrm{~mL}$ of acetate buffer ( $40 \mathrm{mM}$ sodium acetate, $60 \mathrm{mM}$ acetic acid) and $0.2 \mathrm{~mL}$ of enzymes solution ( 3 units of amyloglucosidase and 0.25 units of $\alpha$-amylase); $0.5 \mathrm{~mL}$ of the supernatant was mixed with $0.5 \mathrm{~mL}$ of water and $1 \mathrm{~mL}$ of phenol (5/ $95 ; \mathrm{v} / \mathrm{v}$ ). Thereafter, $5 \mathrm{~mL}$ of concentrated sulfuric acid was added and the solution was left to cool for $15 \mathrm{~min}$. Glucose was measured by its absorbance at $483 \mathrm{~nm}$ and expressed in terms of $\mu \mathrm{g}$ of glucose per $\mathrm{g}$ fresh weight of berry sample. Calibration of the concentration of glucose was performed by determining the absorbance of several concentrations of glucose standards at $483 \mathrm{~nm}(0,20,40$, $\left.80,120,160,200 \mu \mathrm{g} \mathrm{ml}^{-1}\right)$.

\section{Authors' contributions}

LGD conceived the experimental design, set up mRNA extraction, performed microarray experiments, RT-PCR, GC-MS, and starch analyses, prepared figures and tables and wrote the initial manuscript draft. JG performed database analysis. MDW and GRC acquired physiological data. RLT performed the identification of housekeeping genes. DRQ supervised the GC-MS analysis. CO performed microarray analyses. DAS contributed to meta- bolic profiling studies and edited the manuscript. KAS performed all statistical data analysis and edited the manuscript. JCC and GRC contributed equally to the preparation and finalization of the manuscript and conceived the study. All authors have read and approved the final version of the manuscript.

\section{Additional material}

\section{Additional file 1}

Quality control of Vitis GeneChip ${ }^{\circledR}$ genome arrays. The data provided represent the quality controls and commercial specifications of the 21 arrays used in this study. Slide 1. A) Box plot of raw PM (perfect match) probe intensities before and after RMA normalization. Each color indicates a set of three biological replicates. B) RNA degradation plot for all 21 arrays. All lines have similar shapes and similar variation between highest and lowest points. C) Commercial specifications of the Affymetrix Vitis GeneChip version 1.0.

Click here for file

[http://www.biomedcentral.com/content/supplementary/14712164-8-429-S1.ppt]

\section{Additional file 2}

Extensive list of transcripts differentially expressed along berry development. The data provided represent the lists of transcripts that fulfilled the ANOVA filter. Table 1: List of probe sets that passed the ANOVA filter. Table 2: List of Unigenes that passed the ANOVA filter. Table 3: List of probe sets that passed the two-fold ratio (TFR) or greater filter for transcript abundance changes between two stages over berry development. Table 4: List of Unigenes according to Profile number that passed the twofold ratio (TFR) or greater filter for transcript abundance changes between two stages over berry development.

Click here for file

[http://www.biomedcentral.com/content/supplementary/14712164-8-429-S2.xls]

\section{Additional file 3}

Principal component analysis of transcriptomic behavior during grape berry development. Hybridization data from each biological replicate were projected as two graphs according to the A) first and second and B) second and third principal components arranged in descending order of variance. These first three principal components allowed clear distinction of the seven developmental stages with spots representing data from each biological replicate: E-L stage 31 (light green), 32 (dark green), 33 (brown), 34 (burgundy), 35 (yellow), 36 (light purple), and 38 (orange). Analysis was performed using GeneANOVA software [118]. Click here for file

[http://www.biomedcentral.com/content/supplementary/14712164-8-429-S3.ppt] 


\section{Additional file 4}

Template profiles used for PTM analysis. The data provided represent the schematic trends of transcript profiles across berry development used for defining the template profiles. Phases are indicated as I, II, or III. Numbers indicated E-L stages 31 to 38. Pink shading indicates véraison (E-L stages 34 to 35 ).

Click here for file

[http://www.biomedcentral.com/content/supplementary/1471-

2164-8-429-S4.ppt]

\section{Additional file 5}

Supplemental data related to the functional analyses of the Unigenes and to real-time RT-PCR. The data provided represent supplemental data related to Figures 3 and 10. Table 1. Attribution of the 20 profiles to Phase I, II or III according to criteria cited in Methods. Table 2. p-values of the Chi-squared tests of distribution of Unigenes within the three main phases of berry development (I, II and III) for each functional category. Differences in distribution considered as significant are indicated by orange shading. Only Unigenes clustered into the 20 PTM profiles were used for this analysis. Table 3. A list of primers used for quantitative realtime RT-PCR experiments.

Click here for file

[http://www.biomedcentral.com/content/supplementary/14712164-8-429-S5.doc]

\section{Acknowledgements}

This work was supported by grants from the National Science Foundation (NSF) Plant Genome program (DBI-02 17653) to G.R.C., J.C.C., and D.A.S. and the Bioinformatics program (DBI-013656I) to K.A.S. The Nevada Genomics and Proteomics Centers are supported by grants from the NIH Biomedical Research Infrastructure Network (NIH-NCRR, P20 RRI6464) and NIH IDeA Network of Biomedical Research Excellence (INBRE, RR03-008). This research was supported, in part, by the Nevada Agricultural Experiment Station, publication \# 03077039. The authors are indebted to Rebecca Albion and Kitty Spreeman for their invaluable technical support. The authors would like to especially thank Leon Sobon of Sobon Estate and Shenandoah Vineyards, Amador County, California for allowing us to collect the berry samples used in this study.

\section{References}

I. FAOSTAT database (http://faostat.fao.org). Food and Agriculture Organization of the United Nations; 2007.

2. Wine Institute [http://www.wineinstitute.org/]

3. [http://www.wineinstitute.org/industry/statistics/2006/ ca wine economic impact.php].

4. German JB, Walzem RL: The health benefits of wine. Annu Rev Nutr 2000, 20:56I-593.

5. Wollin SD, Jones PJ: Alcohol, red wine and cardiovascular disease. I Nutr 200 I, I 3 I: | 40|-| 404.

6. Bradamante S, Barenghi L, Villa A: Cardiovascular protective effects of resveratrol. Cardiovasc Drug Rev 2004, 22: I69-188.

7. Bruno R, Ghisolfi L, Priulla M, Nicolin A, Bertelli A: Wine and tumors: study of resveratrol. Drugs Exp Clin Res 2003, 29:257-261.

8. Baur JA, Sinclair DA: Therapeutic potential of resveratrol: the in vivo evidence. Nat Rev Drug Discov 2006, 5:493-506.

9. Dore S: Unique properties of polyphenol stilbenes in the brain: more than direct antioxidant actions; gene/protein regulatory activity. Neurosignals 2005, 14:61-70.

10. Jones SB, DePrimo SE, Whitfield ML, Brooks JD: Resveratrolinduced gene expression profiles in human prostate cancer cells. Cancer Epidemiol Biomarkers Prev 2005, 14:596-604.

II. Giovannoni J]: Genetic regulation of fruit development and ripening. Plant Cell 2004, 16 Suppl:SI70-80.
12. Given NK, Venis MA, Gierson D: Hormonal regulation of ripening in the strawberry, a non-climacteric fruit. Planta 1988 , 174:402-406.

13. Coombe BG: Research on development and ripening of the grape berry. Am J Enol Vitic 1992, 43:101-II0.

14. Coombe BG: Adoption of a system for identifying grapevine growth stages. Aust J Grape Wine Res 1995, I: 100-1 I0.

15. Noordeloos S, Nagel CW: Effect of sugar on acid perception in wine. Am J Enol Vitic 1972, 23:। 39- 143.

16. Davies C, Robinson SP: Differential screening indicates a dramatic change in mRNA profiles during grape berry ripening. Cloning and characterization of cDNAs encoding putative cell wall and stress response proteins. Plant Physiol 2000, 122:803-8|2.

17. Waters DL, Holton TA, Ablett EM, Lee LS, Henry RJ: cDNA microarray analysis of developing grape (Vitis vinifera cv. Shiraz) berry skin. Funct Integr Genomics 2005, 5:40-58.

18. Terrier N, Glissant D, Grimplet J, Barrieu F, Abbal P, Couture C Ageorges A, Atanassova R, Leon C, Renaudin JP, Dedaldechamp F, Romieu C, Delrot S, Hamdi S: Isogene specific oligo arrays reveal multifaceted changes in gene expression during grape berry (Vitis vinifera L.) development. Planta 2005, 222:832-847.

19. Fernandez L, Torregrosa L, Terrier N, Sreekantan L, Grimplet J, Davies C, Thomas MR, Romieu C, Ageorges A: Identification of genes associated with flesh morphogenesis during grapevine fruit development. Plant Mol Biol 2007, 63:307-323.

20. Waters DLE, Holton TA, Ablett EM, Slade Lee L, Henry RJ: The ripening wine grape berry skin transcriptome. Plant Sci 2006, 171:132-138.

2I. Irizarry RA, Hobbs B, Collin F, Beazer-Barclay YD, Antonellis KJ, Scherf U, Speed TP: Exploration, normalization, and summaries of high density oligonucleotide array probe level data. Biostatistics 2003, 4:249-264.

22. Benjamini $Y$, Hochberg Y: Controlling the false discovery rate: a practical and powerful approach to multiple testing. J Roy Stat Soc Ser B (Stat Method) 1995, 57:289-300.

23. DFCl Grape Gene Index [http://biocomp.dfci.harvard.edu/tgi/cgibin/tgi/gimain.pl?gudb=grape]

24. Pavlidis P, Noble WS: Analysis of strain and regional variation in gene expression in mouse brain. Genome Biol 200I, 2:research0042.1-research0042.15.

25. Schoof H, Ernst R, Nazarov V, Pfeifer L, Mewes HW, Mayer KF: MIPS Arabidopsis thaliana Database (MAtDB): an integrated biological knowledge resource for plant genomics. Nucleic Acids Res 2004, 32(Database issue):D373-6.

26. Altman A, Kaur-Sawhney R, Galston AW: Stabilization of oat leaf protoplasts through polyamine-mediated inhibition of senescence. Plant Physiol 1977, 60:570-574.

27. Egea-Cortines M, Mizrahi Y: Polyamines in cell division, fruit set and development and seed germination. In Biochemistry and physiology of polyamines in plants Edited by: Slocum RD, Flores HE. Boca Raton, CRC Press; 1991:143-159.

28. Geny L, Broquedis M, Martin TJ, Bouard J: Free, conjugated, and wall-bound polyamines in various organs of fruiting cuttings of Vitis vinifera L. cv. Cabernet Sauvignon. Am J Enol Vitic 1997, 48:80-84.

29. Burtin D, Martin-Tanguy J, Paynot M, Rossin N: Effects of the suicide inhibitors of arginine and ornithine decarboxylase activities on organogenesis, growth, free polyamine and hydroxycinnamoyl putrescine levels in leaf explants of Nicotiana xanthi n.c. cultivated in vitro in a medium producing callus formation. Plant Physiol 1989, 89: 104-1 I0.

30. Sieciechowicz KA, Joy KW, Ireland RJ: The metabolism of asparagine in plants. Phytochemistry 1988, 27:663-67।.

31. Miflin BJ, Habash DZ: The role of glutamine synthetase and glutamate dehydrogenase in nitrogen assimilation and possibilities for improvement in the nitrogen utilization of crops. J Exp Bot 2002, 53:979-987.

32. Nunan KJ, Sims IM, Bacic A, Robinson SP, Fincher GB: Changes in cell wall composition during ripening of grape berries. Plant Physiol 1998, I I 8:783-792.

33. Thomas TR, Matthews MA, Shackel KA: Direct in situ measurement of cell turgor in grape (Vitis vinifera L.) berries during development and in response to plant water deficits. Plant Cell Environ 2006, 29:993-100I. 
34. Brummell DA, Harpster MH: Cell wall metabolism in fruit softening and quality and its manipulation in transgenic plants. Plant Mol Biol 2001, 47:3 I I-340.

35. Saladie M, Rose JK, Cosgrove DJ, Catala C: Characterization of a new xyloglucan endotransglucosylase/hydrolase (XTH) from ripening tomato fruit and implications for the diverse modes of enzymic action. Plant J 2006, 47:282-295.

36. Sampedro J, Cosgrove DJ: The expansin superfamily. Genome Biol 2005, 6:242

37. Dotto MC, Martinez GA, Civello PM: Expression of expansin genes in strawberry varieties with contrasting fruit firmness. Plant Physiol Biochem 2006, 44:301-307.

38. Lund ST, Bohlmann J: The molecular basis for wine grape quality--a volatile subject. Science 2006, 3 I I :804-805

39. Lucker J, Bowen P, Bohlman J: Vitis vinifera terpenoid cyclases: functional identification of two sesquiterpene synthase cDNAs encoding (+)-valencene synthase and (-)-germacrene $D$ synthase and expression of mono- and sesquiterpene synthases in grapevine flowers and berries. Phytochemistry 2004, 65:2649-2659.

40. Martin DM, Bohlman J: Identification of Vitis vinifera (-)-alphaterpineol synthase by in silico screening of full-length cDNA ESTs and functional characterization of recombinant terpene synthase. Phytochemistry 2004, 65:1223-1229.

41. Mahmoud SS, Croteau RB: Strategies for transgenic manipulation of monoterpene biosynthesis in plants. Trends Plant Sci 2002, 7:366-373

42. Jackson RS: Wine Science: Principles, Practice, Perception. In Food Science and Technology International Series 2nd edition. Edited by: Taylor SL. San Diego, Academic Press; 2000.

43. Farina L, Boido E, Carrau F, Versini G, Dellacassa E: Terpene compounds as possible precursors of 1,8 -cineole in red grapes and wines. J Agric Food Chem 2005, 53:1633-1636.

44. Ringer KL, Davis EM, Croteau R: Monoterpene metabolism Cloning, expression, and characterization of (-)-isopiperitenol/(-)-carveol dehydrogenase of peppermint and spearmint. Plant Physiol 2005, I 37:863-872.

45. Davies C, Boss PK, Robinson SP: Treatment of grape berries, a nonclimacteric fruit with a synthetic auxin, retards ripening and alters the expression of developmentally regulated genes. Plant Physiol 1997, I I 5: I I55-I I6I.

46. Chervin C, Tira-Umphon A, Roustan JP, Lamon J, El-Kereamy A Kanellis $A$ : Ethylene is required for the ripening of grape. Acto Hort 2005, 689:25I-256.

47. El-Kereamy A, Chervin C, Roustan JP, Cheynier V, Souquet JM, Moutounet M, Raynal J, Ford C, Latche A, Pech JC, Bouzayen M: Exogenous ethylene stimulates the long-term expression of genes related to anthocyanin biosynthesis in grape berries. Physiol Plant 2003, I | 9: |75-182.

48. Tesniere C, Pradal M, El-Kereamy A, Torregrosa L, Chatelet P, Rous$\tan$ JP, Chervin C: Involvement of ethylene signalling in a nonclimacteric fruit: new elements regarding the regulation of ADH expression in grapevine. J Exp Bot 2004, 55:2235-2240.

49. Hale CR, Coombe BG, Hawker JS: Effects of ethylene and 2-chloroethylphosphonic acid on the ripening of grapes. Plant Physiol 1970, 45:620-623.

50. Chen YF, Etheridge N, Schaller GE: Ethylene signal transduction. Ann Bot (Lond) 2005, 95:901-915.

5I. Klee H, Tieman D: The tomato ethylene receptor gene family Form and function. Physiol Plant 2002, I I 5:336-34 I.

52. Trainotti L, Pavanello A, Casadoro G: Different ethylene receptors show an increased expression during the ripening of strawberries: does such an increment imply a role for ethylene in the ripening of these non-climacteric fruits? I Exp Bot 2005, 56:2037-2046.

53. Symons GM, Davies C, Shavrukov Y, Dry IB, Reid JB, Thomas MR: Grapes on steroids. Brassinosteroids are involved in grape berry ripening. Plant Physiol 2006, I40:I50-I58.

54. Belkhadir $Y$, Chory J: Brassinosteroid signaling: a paradigm for steroid hormone signaling from the cell surface. Science 2006 314:|4|0-14||.

55. Cawthon D, Morris ]: Relationship of seed number and maturity to berry development, fruit maturation, hormona changes and uneven ripening of "Concord" (Vitis labrusca L.) grapes. J Am Soc Hort Sci 1982, I 07: 1097-I I 04.
56. Perez FJ, Viani C, Retamales J: Bioactive gibberellins in seeded and seedless grapes: Identification and changes in content during berry development. Am J Enol Vitic 2000, 5 I:3 I5-3 I 8.

57. Costantini E, Landi L, Silvestroni O, Pandolfini T, Spena A, Mezzetti B Auxin synthesis-encoding transgene enhances grape fecundity. Plant Physiol 2007, I43: 1689-1694.

58. Bartel B, Fink GR: ILRI, an amidohydrolase that releases active indole-3-acetic acid from conjugates. Science 1995, 268: $1745-1748$.

59. Hampel D, Mosandl A, Wust M: Induction of de novo volatile terpene biosynthesis via cytosolic and plastidial pathways by methyl jasmonate in foliage of Vitis vinifera L. J Agric Food Chem 2005, 53:2652-2657.

60. Tassoni A, Fornale S, Franceschetti M, Musiani F, Michael AJ, Perry B, Bagni $\mathrm{N}$ : Jasmonates and Na-orthovanadate promote resveratrol production in Vitis vinifera cv. Barbera cell cultures. New Phytol 2005, 166:895-905.

6I. Schaller F, Biesgen C, Mussig C, Altmann T, Weiler EW: I 2-Oxophytodienoate reductase 3 (OPR3) is the isoenzyme involved in jasmonate biosynthesis. Planta 2000, 21 0:979-984.

62. Werner T, Kollmer I, Bartrina I, Holst K, Schmulling T: New insights into the biology of cytokinin degradation. Plant Biol (Stuttg) 2006, 8:37|-38I.

63. Hepler PK: Calcium: a central regulator of plant growth and development. Plant Cell 2005, I 7:2 |42-2I55.

64. Yu XC, Li MJ, Gao GF, Feng HZ, Geng XQ, Peng CC, Zhu SY, Wang $X$, Shen $Y Y$, Zhang DP: Abscisic acid stimulates a calciumdependent protein kinase in grape berry. Plant Physiol 2006, 140:558-579.

65. Cerana M, Bonza MC, Harris R, Sanders D, De Michelis MI: Abscisic acid stimulates the expression of two isoforms of plasma membrane Ca2+-ATPase in Arabidopsis thaliana seedlings. Plant Biol (Stuttg) 2006, 8:572-578.

66. Cheng NH, Pittman JK, Shigaki T, Lachmansingh J, LeClere S, Lahner $B$, Salt DE, Hirschi KD: Functional association of Arabidopsis CAXI and CAX3 is required for normal growth and ion homeostasis. Plant Physiol 2005, I 38:2048-2060.

67. Coombe BG, Hale CR: The hormone content of ripening grape berries and the effects of growth substance treatments. Plant Physiol 1973, 5 I:629-634.

68. McCormack E, Tsai YC, Braam J: Handling calcium signaling: Arabidopsis CaMs and CMLs. Trends Plant Sci 2005, I 0:383-389.

69. Anandalakshmi R, Marathe R, Ge X, Herr JM Jr., Mau C, Mallory A, Pruss G, Bowman L, Vance VB: A calmodulin-related protein that suppresses posttranscriptional gene silencing in plants. Science 2000, 290: |42-|44.

70. Fagard M, Boutet S, Morel JB, Bellini C, Vaucheret H: AGO I, QDE2 , and RDE-I are related proteins required for post-transcriptional gene silencing in plants, quelling in fungi, and RNA interference in animals. Proc Natl Acad Sci U S A 2000, 97: I1650-11654

7I. Grimplet J, Deluc LG, Tillett RL, Wheatley MD, Schlauch KA, Cramer GR, Cushman JC: Tissue-specific mRNA expression profiling in grape berry tissues. BMC Genomics 2007, 8: 187.

72. Kitamura S, Shikazono N, Tanaka A: TRANSPARENT TESTA 19 is involved in the accumulation of both anthocyanins and proanthocyanidins in Arabidopsis. Plant J 2004, 37: I04- I I 4.

73. Goodman CD, Casati P, Walbot V: A multidrug resistance-associated protein involved in anthocyanin transport in Zea mays. Plant Cell 2004, 16:1812-1826.

74. Alfenito MR, Souer E, Goodman CD, Buell R, Mol J, Koes R, Walbot $V$ : Functional complementation of anthocyanin sequestration in the vacuole by widely divergent glutathione S-transferases. Plant Cell 1998, 10:1 135-1149.

75. Debeaujon I, Peeters AJ, Leon-Kloosterziel KM, Koornneef M: The transparent testa 12 gene of arabidopsis encodes a multidrug secondary transporter-like protein required for flavonoid sequestration in vacuoles of the seed coat endothelium. Plant Cell 200I, I 3:853-872.

76. Walker AR, Lee E, Bogs J, McDavid DA, Thomas MR, Robinson SP White grapes arose through the mutation of two similar and adjacent regulatory genes. Plant J 2007, 49:772-785.

77. Bogs J, Jaffe FW, Takos AM, Walker AR, Robinson SP: The grapevine transcription factor VvMYBPAI regulates proanthocyanidin synthesis during fruit development. Plant Physiol 2007 |43(3): |347-|36| 
78. Deluc L, Barrieu F, Marchive C, Lauvergeat V, Decendit A, Richard T, Carde JP, Merillon JM, Hamdi S: Characterization of a grapevine R2R3-MYB transcription factor that regulates the phenylpropanoid pathway. Plant Physiol 2006, I 40:499-5II.

79. Kobayashi S, Ishimaru M, Hiraoka K, Honda C: Myb-related genes of the Kyoho grape (Vitis labruscana) regulate anthocyanin biosynthesis. Planta 2002, 2 I 5:924-933.

80. Kobayashi S, Goto-Yamamoto N, Hirochika H: Retrotransposoninduced mutations in grape skin color. Science 2004, 304(5673):982

81. Goodrich J, Carpenter R, Coen ES: A common gene regulates pigmentation pattern in diverse plant species. Cell 1992 , 68:955-964.

82. Luan F, Wüst M: Differential incorporation of I-deoxy-D-xylulose into (3S)-linalool and geraniol in grape berry exocarp and mesocarp. Phytochemistry 2002, 60:45I-459.

83. Wu S, Watanabe N, Mita S, Dohra H, Ueda Y, Shibuya M, Ebizuka Y: The key role of phloroglucinol O-methyltransferase in the biosynthesis of Rosa chinensis volatile 1,3,5-trimethoxybenzene. Plant Physiol 2004, I 35:95-102.

84. Ross JR, Nam KH, D'Auria JC, Pichersky E: S-Adenosyl-L-methionine:salicylic acid carboxyl methyltransferase, an enzyme involved in floral scent production and plant defense, represents a new class of plant methyltransferases. Arch Biochem Biophys 1999, 367:9-16.

85. Pichersky E, Noel JP, Dudareva N: Biosynthesis of plant volatiles: nature's diversity and ingenuity. Science 2006, 3 I I:808-8 I I.

86. DeBolt S, Cook DR, Ford CM: L-tartaric acid synthesis from vitamin C in higher plants. Proc Natl Acad Sci U S A 2006 I 03:5608-56/3.

87. Possner DRE, Kliewer WM: The localization of acids, sugars, potassium and calcium in developing grape berries. Vitis 1985 24:229-240.

88. Stines AP, Naylor DJ, Hoj PB, Van Heeswijck R: Proline accumulation in developing grapevine fruit occurs independently of changes in the levels of Delta I-pyrroline-5-carboxylate synthetase mRNA or protein. Plant Physiol 1999, I 20:923-931.

89. Stines AP, Grubb J, Gockowiak H, Henschke PA, Hoj PB, Heeswijck $\mathrm{RV}$ : Proline and arginine accumulation in developing berries of $\mathbf{V}$. vinifera in Australian vineyards: Influence of vine cultivar, berry maturity and tissue type. Aust J Grape Wine Res 2000 , 6: $150-158$.

90. Conde C, Silva P, Fontes N, Dias A, Tavares R, Sousa M, Agasse A Delrot S, Geros $\mathrm{H}$ : Biochemical changes throughout grape berry development and fruit and wine quality. Food 2007 I: $1-22$

91. Swanson CA, El-Shishiny ED: Translocation of sugars in the Concord grape. Plant Physiol 1958, 33:33-37.

92. Conde C, Agasse A, Glissant D, Tavares R, Geros H, Delrot S: Pathways of glucose regulation of monosaccharide transport in grape cells. Plant Physiol 2006, I41: I563-I577.

93. Komatsu A, Moriguchi T, Koyama K, Omura M, Akihama T: Analysis of sucrose synthase genes in citrus suggests different roles and phylogenetic relationships. J Exp Bot 2002, 53:6I-7I.

94. Davies C, Robinson SP: Sugar accumulation in grape berries: cloning for two putative vacuolar invertase cDNAs and their expression in grapevine tissue. Plant Physiol 1996, I I I:275-283.

95. Zhang XY, Wang XL, Wang XF, Xia GH, Pan QH, Fan RC, Wu FQ Yu XC, Zhang DP: A shift of phloem unloading from symplasmic to apoplasmic pathway is involved in developmental onset of ripening in grape berry. Plant Physiol 2006, 142:220-232

96. Geigenberger $P$, Stitt $M$ : Sucrose synthase catalyses a readily reversible reaction in vivo developing potato tubers and other plant tissues. Planta 1993, 189:329-393.

97. Koch KE: Carbohydrate-modulated gene expression in plants. Annu Rev Plant Physiol Plant Mol Biol 1996, 47:509-540.

98. Ruan Y: Rapid cell expansion and cellulose synthesis regulated by plasmodesmata and sugar: insights from the single-celled cotton fibre. Funct Plant Biol 2007, 34: I-I0.

99. Vignault C, Vachaud M, Cakir B, Glissant D, Dedaldechamp F, Buttner M, Atanassova R, Fleurat-Lessard P, Lemoine R, Delrot S: VvHT I encodes a monosaccharide transporter expressed in the conducting complex of the grape berry phloem. I Exp Bot 2005, 56: |409-|4|8.
100. Davies C, Wolf T, Robinson SP: Three putative sucrose transporters are differentially expressed in grapevine tissues. Plant Sci 1999, 147:93-100.

I0I. Klepek YS, Geiger D, Stadler R, Klebl F, Landouar-Arsivaud L, Lemoine $R$, Hedrich $R$, Sauer $N$ : Arabidopsis POLYOL TRANSPORTER5, a new member of the monosaccharide transporter-like superfamily, mediates $\mathbf{H + - s y m p o r t ~ o f ~}$ numerous substrates, including myo-inositol, glycerol, and ribose. Plant Cell 2005, I 7:204-218.

102. Kore-eda S, Noake C, Ohishi M, Ohnishi J, Cushman JC: Transcriptional profiles of organellar metabolite transporters during induction of crassulacean acid metabolism in Mesembryanthemum crystallinum. Funct Plant Biol 2005, 32:45I-466.

103. Gao Z, Maurousset L, Lemoine R, Yoo SD, van Nocker S, Loescher W: Cloning, expression, and characterization of sorbitol transporters from developing sour cherry fruit and leaf sink tissues. Plant Physiol 2003, I 3 I: I 566-I 575.

104. Roubelakis-Angelakis KA, Kliewer WM: The composition of bleeding sap from Thompson Seedless grapevines as affected by nitrogen fertilization. Am J Enol Vitic 1979, 30: |4-I8.

105. Delvalle D, Dumez S, Wattebled F, Roldan I, Planchot V, Berbezy P, Colonna P, Vyas D, Chatterjee M, Ball S, Merida A, D'Hulst C: Soluble starch synthase I: a major determinant for the synthesis of amylopectin in Arabidopsis thaliana leaves. Plant J 2005, 43:398-4I2

106. Li CY, Weiss D, Goldschmidt EE: Effects of carbohydrate starvation on gene expression in citrus root. Planta 2003, 2 I 7: I I-20.

107. Diakou P, Carde JP: In situ fixation of grape berries. Protoplasma 200।, 2 I 8:225-235.

108. Hotta CT, Gardner MJ, Hubbard KE, Baek SJ, Dalchau N, Suhita D, Dodd AN, Webb AA: Modulation of environmental responses of plants by circadian clocks. Plant Cell Environ 2007, 30(3):333-349.

109. Lu Y, Gehan JP, Sharkey TD: Daylength and circadian effects on starch degradation and maltose metabolism. Plant Physio 2005, I 38(4):2280-229|.

I I0. Pocock KF, Sefton MA, Williams PJ: Taste thresholds of phenolic extracts of French and American oakwood: the influence of oak phenols on wine flavor. Am J Enol Vitic 1994, 45(4):429-434.

III. Robinson SP, Jacobs AK, Dry IB: A class IV chitinase is highly expressed in grape berries during ripening. Plant Physiol 1997, I | 4(3):77|-778.

I I2. Jach G, Gornhardt B, Mundy J, Logemann J, Pinsdorf E, Leah R, Schell $J$, Maas $C$ : Enhanced quantitative resistance against fungal disease by combinatorial expression of different barley antifungal proteins in transgenic tobacco. Plant J I995, 8(I):97-109.

I 13. Tattersall DB, Van Heeswijck R, Hoj PB: Identification and characterization of a fruit-specific, thaumatin-like protein that accumulates at very high levels in conjunction with the onset of sugar accumulation and berry softening in grapes. Plant Physiol I 997, I I 4(3):759-769.

I 14. Busam G, Junghanns KT, Kneusel RE, Kassemeyer HH, Matern U: Characterization and expression of caffeoyl-coenzyme A 3 O-methyltransferase proposed for the induced resistance response of Vitis vinifera L. Plant Physiol I997, I I 5(3): I 039- I 048.

115. McCutchan J, Shackel KA: Stem-water potential as a sensitive indicator of water stress in prune trees (Prunus domestica $\mathbf{L}$. cv. French). J Amer Soc Hort Sci 1992, I I 7:607-6I I.

1 16. Tattersall EAR, Ergul A, AIKayal F, DeLuc L, Cushman JC, Cramer GR: Comparison of methods for isolating high-quality RNA from leaves of grapevine. Am J Enol Vitic 2005, 56:400-406.

117. Gautier L, Cope L, Bolstad BM, Irizarry RA: affy--analysis of Affymetrix GeneChip data at the probe level. Bioinformatics 2004, 20:307-315.

I 18. Didier G, Brezellec P, Remy E, Henaut A: GeneANOVA--gene expression analysis of variance. Bioinformatics 2002, I 8:490-49|.

119. Shen L, Gong J, Caldo RA, Nettleton D, Cook D, Wise RP, Dickerson JA: BarleyBase--an expression profiling database for plant genomics. Nucleic Acids Res 2005, 33(Database Issue):D6|4-D6|8.

120. Saeed Al, Sharov V, White J, Li J, Liang W, Bhagabati N, Braisted J, Klapa M, Currier T, Thiagarajan M, Sturn A, Snuffin M, Rezantsev A Popov D, Ryltsov A, Kostukovich E, Borisovsky I, Liu Z, Vinsavich A, Trush V, Quackenbush J: TM4: a free, open-source system for microarray data management and analysis. BioTechniques 2003, 34:374-378. 
I2I. Chernoff H, Lehman EL: The use of maximum likelihood estimates in chi2 tests for goodness-of-fit. Ann Math Stat 1954, 25:579-586.

122. Rozen S, Skaletsky H: Primer3 on the WWW for general users and for biologist programmers. Methods Mol Biol 2000, 132:365-386.

123. Luu-The V, Paquet N, Calvo E, Cumps J: Improved real-time RTPCR method for high-throughput measurements using second derivative calculation and double correction. BioTechniques 2005, 38:287-293.

124. Vandesompele J, De Preter K, Pattyn F, Poppe B, Van Roy N, De Paepe A, Speleman F: Accurate normalization of real-time quantitative RT-PCR data by geometric averaging of multiple internal control genes. Genome Biol 2002, 3:RESEARCH0034.

125. Broeckling CD, Huhman DV, Farag MA, Smith JT, May GD, Mendes P, Dixon RA, Sumner LW: Metabolic profiling of Medicago truncatula cell cultures reveals the effects of biotic and abiotic elicitors on metabolism. J Exp Bot 2005, 56:323-336.

126. NIST Standard Reference Database [http://www.nist.gov/srd/]. National Institute of Standards and Technology

127. Kopka J, Schauer N, Krueger S, Birkemeyer C, Usadel B, Bergmuller E, Dormann P, Weckwerth W, Gibon Y, Stitt M, Willmitzer L, Fernie AR, Steinhauser D: GMD@CSB.DB: the Golm Metabolome Database. Bioinformatics 2005, 21:1635-1638.

128. Dubois M, Gilles K, Hamilton J, Rebers P, Smith F: Colorimetric method for the determination of sugars and related substances. Anal Chem 1956, 28:350-356.

Publish with Biomed Central and every scientist can read your work free of charge

"BioMed Central will be the most significant development for disseminating the results of biomedical research in our lifetime. "

Sir Paul Nurse, Cancer Research UK

Your research papers will be:

- available free of charge to the entire biomedical community

- peer reviewed and published immediately upon acceptance

- cited in PubMed and archived on PubMed Central

- yours - you keep the copyright

Submit your manuscript here:

http://www.biomedcentral.com/info/publishing_adv.asp
BioMedcentral 